\title{
RACE-OC project: rotation and variability in the open cluster NGC 2099 (M 37)
}

\author{
S. Messina ${ }^{1}$, E. Distefano ${ }^{1}$, P. Parihar ${ }^{2}$, Y. B. Kang ${ }^{3,4,5}$, S.-L. Kim ${ }^{4}$, S.-C. Rey ${ }^{3}$, and C.-U. Lee $^{4}$ \\ 1 INAF-Catania Astrophysical Observatory, via S. Sofia 78, 95123 Catania, Italy \\ e-mail: [sergio.messina; elisa.distefano]@oact.inaf.it \\ 2 Indian Institute of Astrophysics, Block II, Koramangala, Bangalore 560034, India \\ e-mail: psp@iiap.res.in \\ 3 Department of Astronomy and Space Science, Chungnam National University, Daejeon, Korea \\ 4 Korea Astronomy and Space Science Institute, Daejeon, Korea \\ 5 Department of Physics and Astronomy, Johns Hopkins University, Baltimore, MD 21218, USA
}

Received 7 December 2007 / Accepted 26 February 2008

\section{ABSTRACT}

\begin{abstract}
Context. Rotation and solar-type magnetic activity are closely related to each other in main-sequence stars of $\mathrm{G}$ or later spectral types. The presence and level of magnetic activity depend on star's rotation, and rotation itself is strongly influenced by strength and topology of the magnetic fields. Open clusters represent especially useful targets to investigate the connection between rotation and activity.

Aims. The open cluster NGC 2099 has been studied as a part of the RACE-OC project (Rotation and ACtivity Evolution in Open Clusters), which is aimed at exploring the evolution of rotation and magnetic activity in the late-type members of open clusters of different ages.

Methods. We collected time series CCD photometric observations of this cluster in January 2004, and we determined the presence of periodicities in the flux variation related to the stellar rotation by Fourier analysis. We investigate the relations between activity manifestations, such as the light curve amplitude, and global stellar parameters.

Results. We have discovered 135 periodic variables, 122 of which are candidate cluster members. Determination of rotation periods of G- and K-type stars has allowed us to better explore the evolution of angular momentum at an age of about $500 \mathrm{Myr}$. In our analysis, we have also identified 3 new detached eclipsing binary candidates among cluster members.

Conclusions. A comparison with the older Hyades cluster ( $625 \mathrm{Myr})$ shows that the newly-determined distribution of rotation periods is consistent with the scenario of rotational braking of main-sequence spotted stars as they age. However, a comparison with the younger M 34 cluster ( $200 \mathrm{Myr}$ ) shows that the G8-K5 members of these clusters have the same rotation period distribution. That is, G8-K5 members in NGC 2099 seem to have experienced no significant braking in the age range from $\sim 200$ to 500 Myr. Finally, NGC 2099 members have a smaller level of photospheric magnetic activity, as measured by light curve amplitude, than in younger stars of the same mass and rotation, suggesting that the activity level also depends on some other age-dependent parameters.
\end{abstract}

Key words. stars: activity - stars: binaries: close - stars: late-type - stars: rotation - stars: starspots -

Galaxy: open clusters and associations: individual: NGC 2099

\section{Introduction}

The RACE-OC project, which stands for Rotation and ACtivity Evolution in Open Clusters, is a long-term project aimed at studying the evolution of the rotational properties and the magnetic activity of late-type members of stellar open clusters (Messina 2007). Stellar open clusters represent privileged astrophysical targets since they provide complete and homogeneous stellar samples to explore a variety of relevant problems of astrophysical impact. The homogeneity of the stellar sample arises from the fact that all cluster members were formed in similar environmental conditions, characterised by same age as well as initial chemical composition, and are subjected to same interstellar reddening. Such complete stellar samples allow us to accurately

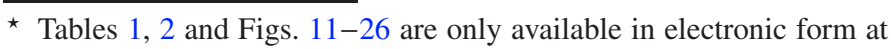
www . aanda.org

$\star \star$ Photometric data of periodic variables are only available in electronic form at the CDS via anonymous ftp to cdsarc.u-strasbg. fr $(130.79 .128 .5)$ or via

http://cdsweb.u-strasbg.fr/cgi-bin/qcat?J/A+A/483/253 investigate those stellar properties, and their mutual relations, which depend on age and metallicity.

Indeed, rotation is one of the basic stellar properties on which the present project is focused. It undergoes dramatic changes along the whole stellar life, as predicted by evolution models of angular momentum of late-type stars (Kawaler 1988; MacGregor \& Brenner 1991; Krishnamurthi et al. 1997; Bouvier et al. 1997; Sills et al. 2000; Ivanova \& Taam 2003; Holzwarth \& Jardine 2007). However, a growing body of evidence, especially from observational studies of open clusters, shows that a significant discrepancy exists with respect to the theoretical expected scenario. The observed spread of rotation periods among stars of similar mass, age, and chemical composition (see e.g., Rebull et al. 2004), the existence of slowly rotating Pre-Main-Sequence (PMS) stars (Herbst \& Mundt 2005), and the well-known decay of rotation rate shown by main-sequence stars when they age at approximately constant radius (see e.g., Barnes 2003), are just a few examples of the mentioned discrepancy with respect to predictions of evolutionary models. 
It is believed that strong magnetic fields play a fundamental role in altering the rotational properties of late-type stars. They are responsible for angular momentum loss or its internal redistribution, and represent a powerful tool to probe the stellar internal structure. For example, magnetic fields are believed to play a key role in the distribution of the mass moment of inertia by coupling the radiative core with the external convection zone (e.g., Barnes 2003). The study of angular momentum evolution is very important to better understand the magnetic activity phenomena, which manifest themselves in late-type stars and directly depend on stellar rotation. Photospheric cool spots and bright faculae, chromospheric plages, and X-ray emission all arise from the action of an hydromagnetic dynamo whose efficiency is related to the star's rotation rate (e.g., Messina et al. 2001, 2003).

Thus, the study of the evolution of angular momentum and magnetic activity offers a complementary approach to understanding the mechanisms by which rotation and magnetic fields influence each other and, eventually, to better understand the nature of late-type stars.

To date, notwithstanding a number of valuable projects, such as MONITOR (Hodgkin et al. 2006), EXPLORE/OC (Extrasolar Planet Occultation Research; von Braun et al. 2005), and the RCT (Robotically Controlled Telescope) monitoring project (Guinan et al. 2003), which are rapidly increasing our knowledge of the rotational properties of late-type members of open clusters, the number of studied open clusters, as well as the number of variables in each cluster, have not been large enough to fully constrain the various models proposed to describe the mechanisms that drive the angular momentum evolution. Specifically, the sequence of ages at which the angular momentum evolution has been studied still has significant gaps, and the sample of cluster members for a number of clusters is not as complete as necessary.

The major science drivers of the RACE-OC project are to explore rotational properties of late-type stars in selected open clusters and associated stellar activity. So far, there have not been many studies exploring the age dependences of various manifestations of stellar activity such as spot temperature, total spot area, the spatial distribution of starspots on stellar surfaces, starspot activity cycles, flip-flop phenomena, and surface differential rotation (SDR). Therefore, in comparison to similar ongoing projects, we focussed our attention on the time evolution of stellar magnetic activity.

Keeping these objectives in mind, we have selected open clusters with an age in the range from 1 to 500 Myr (Messina 2007) for which no rotation and activity investigations have been so far carried out. Furthermore, top priority is given to the open clusters that fill the gaps in the empirical description of the ageactivity-rotation relationship. We have also included in our sample clusters that have been already extensively studied such as the Pleiades and the Orion Nebula clusters (Padmakar Parihar et al. 2008). The motivation behind this is to further enrich the sample of periodic variables and to explore the long-term magnetic activity, e.g., to search for activity cycles and SDR, by making repeated observations of same clusters over several years.

Our sample also includes open clusters that were previously monitored with different scientific motivations, but the re-analysis of archived time series data can provide valuable results on late-type stars. This is the case of the $\sim 500-\mathrm{Myr}$ intermediate-age open cluster NGC 2099 (M 37), which is the first cluster of the RACE-OC project for which we present our results. Although it was previously studied to search for earlytype pulsating variables (Kang et al. 2007), the collected observations allowed us to carry out a valuable and accurate study of rotation and magnetic activity. To date, there is no available data on rotation periods of clusters between the age range from 200 to $600 \mathrm{Myr}$ and, hence, the study of NGC 2099 with an age of $\sim 500 \mathrm{Myr}$ is one attempt to fill the age gap while modelling the angular momentum evolution (Barnes 2003, 2007).

NGC 2099 (M 37) is a $~ 500$ Myr intermediate-age open cluster at distance of nearly 1400 parsec. The cluster, containing thousands of members within core radius of 4-6 arcmin, is found to be very suitable for any monitoring program, using intermediate aperture telescope with moderate FoV. The cluster is close to the galactic plane $\left(l \sim 3^{\circ}\right)$ and hence subjected to substantial reddening $E(B-V) \sim 0.2-0.3$ mag. In the recent past, NGC 2099 has been extensively studied by several researchers to obtain global parameters and to characterise its members (Mermilliod et al. 1996; Nilakshi \& Sagar 2002; Hartman et al. 2008a, and references therein). With different motivations, NGC 2099 was also chosen for photometric monitoring by three different groups to search for new variables. The first two variability surveys carried out by Kiss et al. (2001) and Kang et al. (2007) identified only 24 new variables. Whereas, the much deeper and very rigorous Multiple Mirror Telescope (MMT) transit survey, in which several hundreds of variables are identified, has been carried out recently by Hartman et al. (2008b). We started our project on this cluster earlier than the MMT survey and before the Hartman et al. (2008b) detailed work was communicated. Our work on NGC 2099 can be considered valuable due to the following reasons, at least:

1. Stars brighter than 14.5 mag in $r$ band ( $\sim 15$ mag in $V$ band), that is, F- and earlier-type stars, are saturated in MMT survey and hence our bright NGC 2099 F-type variables are the first to be discovered and they help to make the sample of variable stars more complete for any future study.

2. Observing epochs are different (2004 for present study and 2006 for MMT survey) and hence the common variables of both surveys can be utilised to explore variations in light curves, related to magnetic activity.

3. The prime motivation of the present study is not just to identify the new variables in the cluster, but to use them to investigate evolution of angular momentum and stellar activity vs. age.

In Sect. 2, we give information on observations, achieved photometric accuracy, and selection criteria for membership. The search for periodic variables is presented in Sect. 3, and results are given in Sect. 4. Discussion and conclusions are given in Sects. 5 and 6.

\section{Observations and data analysis}

The present study is based on observations taken in January 2004 with the $1.0 \mathrm{~m}$ telescope at the Mt. Lemmon Optical Astronomy Observatory (LOAO) in Arizona (USA), which feeds a $2 \mathrm{~K} \times 2 \mathrm{~K}$ CCD camera. The observed FoV is about $22.2 \times$ $22.2 \operatorname{arcmin}^{2}$ at the f/7.5 Cassegrain focus. We collected a sequence of 581 images in the $V$-band filter with an exposure time of $600 \mathrm{~s}$ over a total time interval of about 30 days. Kang et al. (2007) gave a detailed description of these observations and data reduction. An example image of the observed NGC 2099 field is plotted in Fig. 1, where the overplotted small circles identify the candidate cluster members we discovered to be periodic variables.

The HR diagram of all the stars detected in 600-s exposures of the NGC 2099 field is displayed as dots in Fig. 2. In 


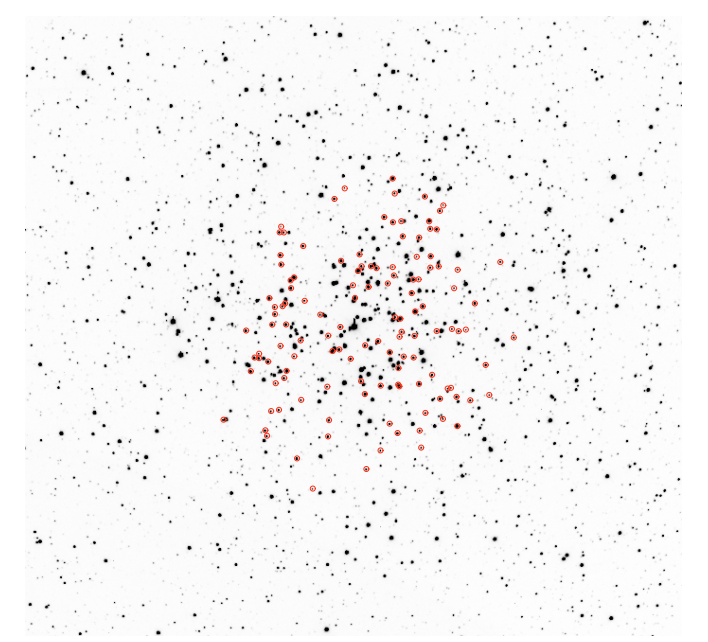

Fig. 1. Observed $V$-band CCD field $\left(22.2 \times 22.2 \operatorname{arcmin}^{2}\right)$ of the open cluster NGC 2099 . Small circles identify the cluster candidate members newly discovered to be periodic variables.

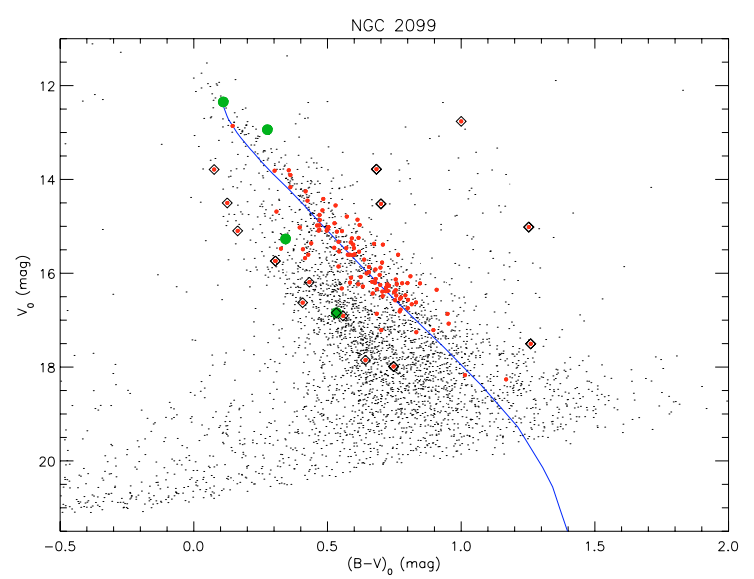

Fig. 2. HR diagram of the stars (dots) detected in the field of NGC 2099. Periodic variables are plotted with red small bullets. A diamond is overplotted to periodic non-cluster members. Large green bullets represent newly-discovered candidate detached eclipsing binaries. The solid line represents the isochrone corresponding to an age of $t=485 \pm 28 \mathrm{Myr}$, $E(B-V)=0.227 \mathrm{mag}$ and $[\mathrm{M} / \mathrm{H}]=0.045$ (Hartman et al. 2008a).

the HR diagram the newly-discovered periodic variable stars are marked by small red bullets. Bullets with an overplotted diamond are non cluster member periodic variables, according to the selection criterion of membership outlined below. The large green bullets show the detached eclipsing binaries newly discovered in our analysis. The solid line is the cluster isochrone. For the present analysis we adopted the following cluster parameters from Hartman et al. (2008a), age $=485 \pm 28 \mathrm{Myr}$, $E(B-V)=0.227 \mathrm{mag},[\mathrm{M} / \mathrm{H}]=0.045$ and distance modulus $(m-M)=11.572 \mathrm{mag}$. The proximity of any star in the HR diagram with respect to the cluster isochrone has been adopted as criterion to select cluster members (see e.g. Irwin 2006, 2008). We selected any star with given intrinsic $(B-V)_{0}$ colour and magnitude difference $\Delta V$ smaller than \pm 1.4 mag with respect to the isochronal magnitude, as a candidate cluster member. This magnitude difference is large enough to include the sequence of binary stars, if any, and to properly take into account the error on dereddened $(B-V)_{0}$ colour. Such a membership selection, based on photometry alone, cannot prevent from a certain level of contamination by non cluster field stars. Hartman et al. (2008a)

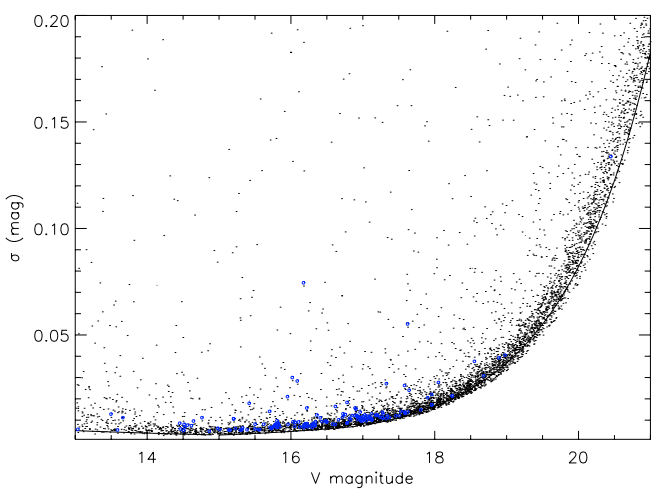

Fig. 3. Standard deviation $\sigma$ of the light curves of the stars detected in the field of NGC 2099 vs. average magnitude. The solid line represents a composite fit to the lower envelope of the $\sigma$ distribution and gives the best achieved photometric accuracy. Small blue bullets represent the periodic variable candidate cluster members.

report such contamination to be on average about $39 \%$. However, as shown in Fig. 1, the periodic variables we discovered are all in the innermost NGC 2099 field region, where the population of cluster members is usually relatively higher and, hence, the percentage of contamination is expected to be, on average, significantly smaller.

The 600-s long exposures of the NGC 2099 field have allowed us to achieve a photometric accuracy $\sigma$ in the $V$ band as good as $0.003 \mathrm{mag}$ in the $13<V<16 \mathrm{mag}$ range, and better than $0.01 \mathrm{mag}$ for all stars brighter than $V \simeq 17.5$ mag. In Fig. 3 we plot the standard deviation of the light curves of all stars detected in the $13<V<21$ mag range vs. their mean $V$ magnitude. The lower envelope of the $\sigma$ distribution is populated by nonvariable and variable stars with least intrinsic variation. The lower envelope gives a measure of the best photometric accuracy we achieved at different magnitudes. Here we would like to point out that the $\sigma$ values are not those automatically computed by DAOPHOT in the PSF magnitude extraction, but time series data of each star were binned with the time interval of $20 \mathrm{~min}$. For each bin we computed the mean magnitude and standard deviation that were, finally, averaged to obtain $\sigma$. The standard deviation computed, following this procedure, is an empirical estimate of effective precision of our photometry. Such an estimate is conservative because the true observational accuracy could be, in principle, even better for stars showing substantial variability within the timescale closer to our fixed binning time interval.

To construct the relation between observational accuracy and magnitude, we followed the procedure given e.g., by Roze \& Hintz (2007), and fitted the lower envelope of Fig. 3 with a piecewise continuous function of the form:

$$
\begin{array}{rlr} 
& =0.1495 \times \mathrm{e}^{-0.26 V} & 13<V \leq 15 \\
\operatorname{accuracy}(\mathrm{mag}) & =3.5 \times 10^{-6} \mathrm{e}^{-0.45 V} & 15<V \leq 17 \\
& =9.2 \times 10^{-9} \mathrm{e}^{-0.80 V} & V>17
\end{array}
$$

Out of a total of 2250 stars detected in the $13<V<20$ mag range, 1746 turned out to be candidate cluster members.

\section{Search for periodicities}

One of the primary goals of the present study is to search for periodicities in the time series photometric magnitudes of all the members of NGC 2099 open cluster. We have limited our investigation to stars in the $13<V<20$ mag range. Stars brighter 

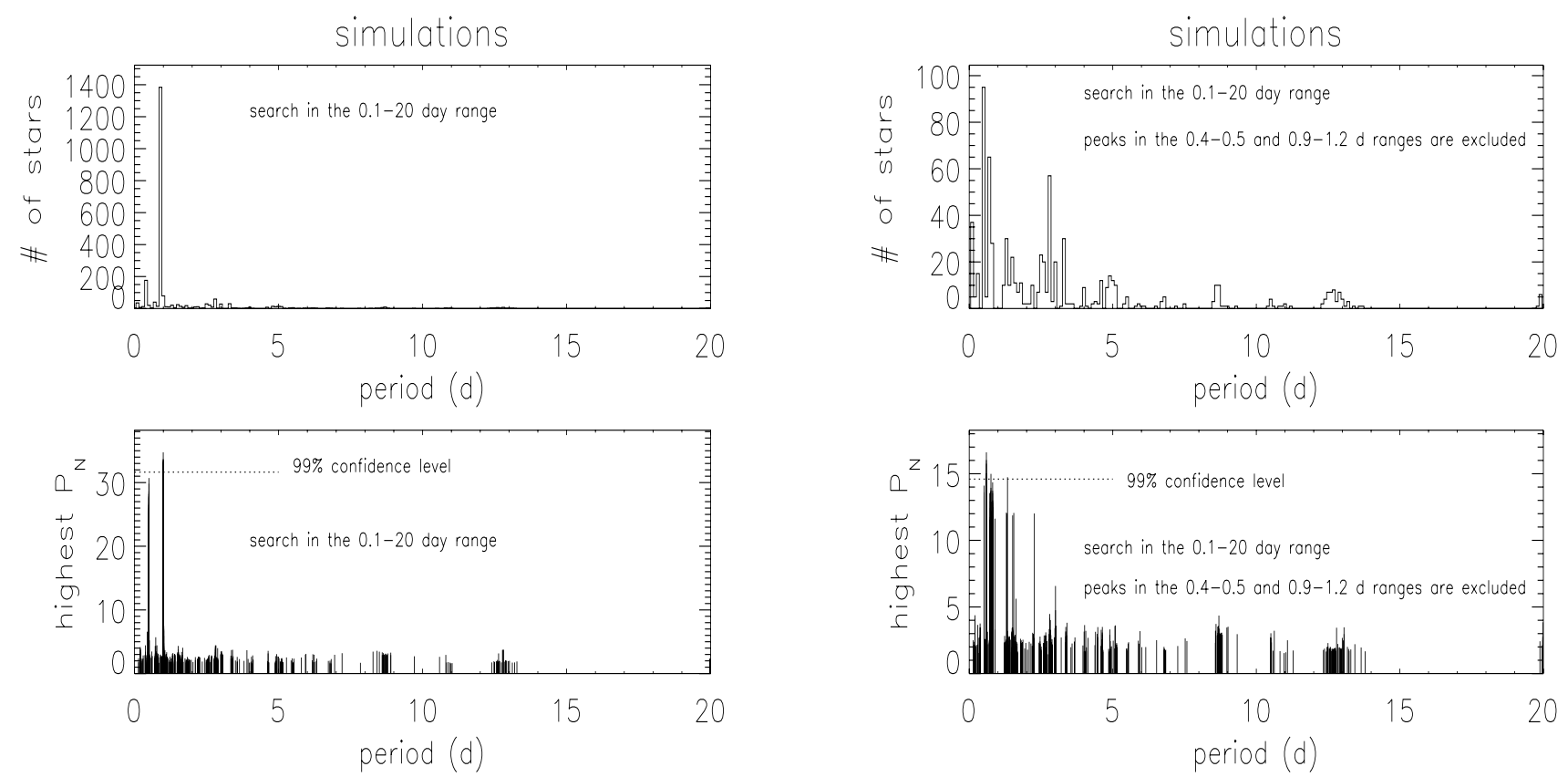

Fig. 4. Left panels: results of periodogram analysis on "randomised" light curves. Right panels: the same as in left panel, but with exclusion of power peaks in the $0.4-0.5$ and $0.9-1.2$ day ranges.

than $V \simeq 13$ are saturated in most of the long-exposure frames, whereas stars fainter than $V \simeq 20$ have a rather low (lower than $0.07 \mathrm{mag}$ ) photometric accuracy to allow us to reliably detect any periodicity, which we will discuss further in Sect. 4.3. We expect that the presence of periodicity in the cluster members of G or later spectral type likely arises from solar-type magnetic activity. Specifically, uneven distributions of cool spots along the stellar longitude, whose visibility is modulated by the star's rotation, give rise to quasi-periodic variation of the observed stellar flux. For these stars, the periodicity indeed represents the star's rotation period. For the candidate cluster members of F spectral type, which are not expected to host magnetic activity, the presence of periodicity may likely arise from pulsations. In these stars, the periodicity indeed represents the period of one of the pulsational modes.

Two different periodogram approaches, the Scargle-Press and the CLEAN periodograms, have been used to search for significant periodicities. In the following sub-sections, we will briefly describe the techniques and the criteria used to classify variable stars as periodic.

\subsection{Scargle-Press periodogram}

The Scargle technique has been developed to search for significant periodicities in unevenly sampled data (Scargle 1982; Horne \& Baliunas 1986). The algorithm calculates the normalised power $P_{\mathrm{N}}(\omega)$ for a given interval of angular frequencies $\omega=2 \pi v$. The highest peak in the calculated periodogram power spectrum reveals the presence and the frequency of a periodicity in the analysed time series data. In order to determine the significance level of the periodic signal, the height of the corresponding power peak is compared with the false alarm probability (FAP).

The FAP is the probability that a peak of given height is due to simply statistical variations, i.e., white noise. This method assumes that each magnitude measurement is independent from the other. However, this is not strictly true for our data time series (see, e.g., Herbst \& Wittenmyer 1996; Stassun et al. 1999) consisting of numerous data consecutively collected within the same night and with a time sampling much shorter than both the periodic or the irregular intrinsic variability timescales we are looking for $\left(P^{\mathrm{d}}=0.1-20\right)$. In order to overcome this problem, we decided to determine the FAP in different way than proposed by Scargle (1982) and Horne \& Baliunas (1986), which is only based on the number of independent frequencies, but following the approach described by Herbst et al. (2002) based on Monte Carlo simulations.

Specifically, we selected a total of 2250 stars whose magnitude is in the $13<V<20$ mag range and which were present in all 581 long-exposure images, thus ensuring an equal time sampling for all the stars under analysis. The magnitude measurements and decimal parts of the Modified Julian Day's of each star's time series were left untouched, while the day numbers were randomly scrambled, thus ensuring that the correlations in the original data is preserved. Then, we applied the periodogram analysis to the "randomised" data series of each star. For each computed periodogram we retained the highest power peak and the corresponding period. In the top-left panel of Fig. 4, we plot the distribution of detected periods from our simulations, whereas in the bottom-left panel we plot the distribution of the highest power peaks vs. period.

The FAP related to a given power $P_{\mathrm{N}}$ is taken as the fraction of randomised light curves that have the highest power peak exceeding $P_{\mathrm{N}}$ which, in turn, is the probability that a peak of this height is due simply statistical variations, i.e. white noise. We find that the power corresponding to a FAP $<0.01$ is $P_{\mathrm{N}}>31.64$. However, we note that such a power threshold is period dependent and, in our specific case, is set by those peaks appearing in the $0.4-0.5$ and $0.9-1.2$ day ranges, which are about $90 \%$ of total detections from simulations. Therefore, $P_{\mathrm{N}}=31.64$ more exactly gives the $99 \%$ level of confidence of a period detected in these mentioned time ranges. A straightforward application of this power level largely underestimates the confidence level of any other period detected outside these ranges with the risk to classify real detections, as they were spurious. Therefore, after removing all peaks falling in these mentioned ranges, we 

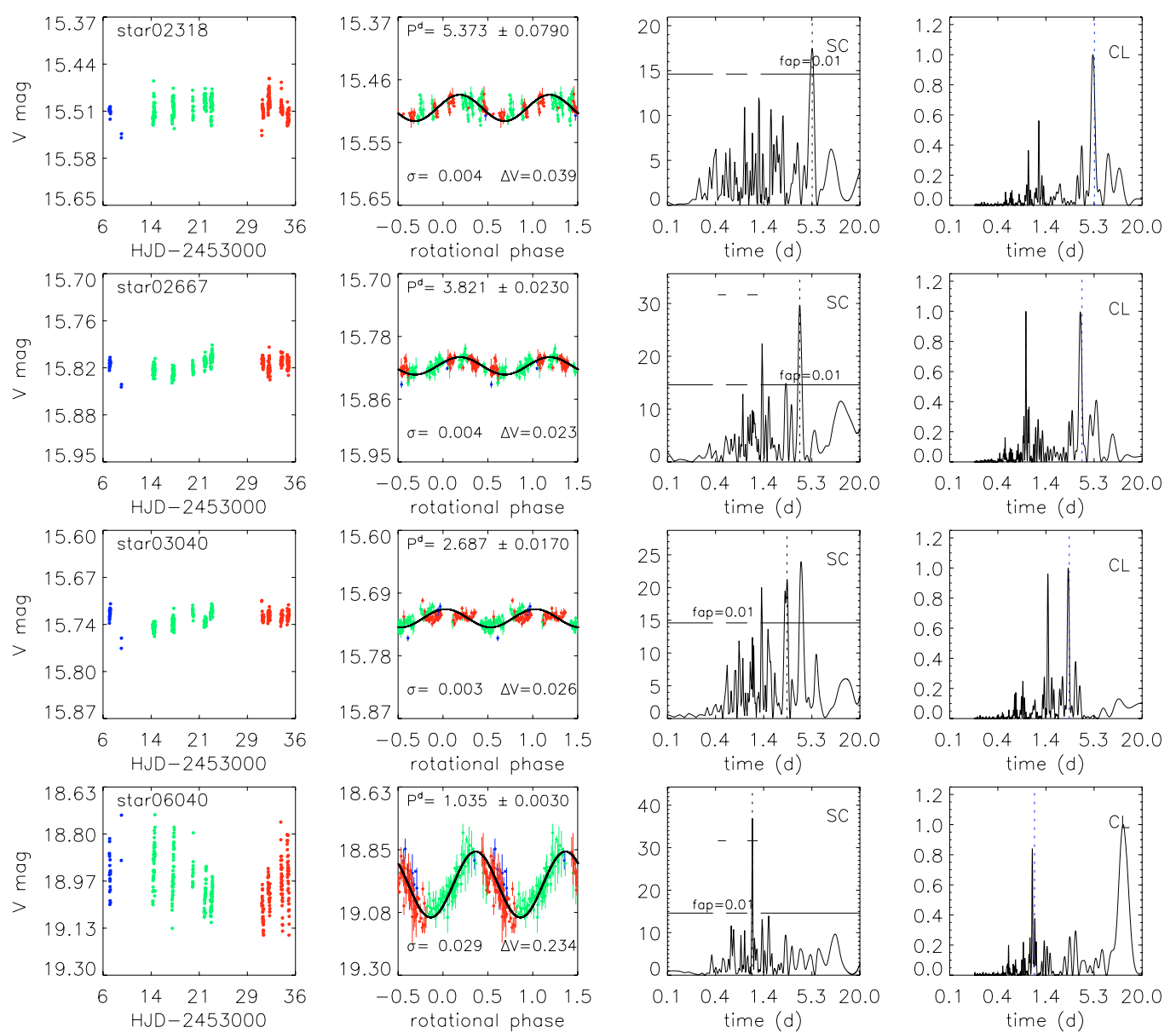

Fig. 5. Example of different results obtained with our period search analysis. Panels show (from left to right) the data time series with colour showing different time intervals; the phased light curve with labelled the rotation period, its uncertainty, the data accuracy, and light curve amplitude. Star 02318: only one period is detected with confidence level larger than 99\% and, independently confirmed by CLEAN analysis. Star 02667: two periods are detected; however, the shorter one is disregarded since it does not reach the power level of $P_{\mathrm{N}}=31.64$ set for the $0.9-1.2$ day range. Star 03040: two periods are detected with confidence level larger than 99\%, however only the highest is confirmed by the CLEAN analysis. Star 06040: the only period detected is in the 0.9-1.2 day range and has a power level larger that the one set for this period range.

recomputed the distribution of power peaks vs. period. The results, which are plotted in the right panels of Fig. 4, show that a $99 \%$ confidence level is reached for power levels $P_{\mathrm{N}}>14.60$. In the following analysis, these two different thresholds will be applied to the corresponding period ranges to select the final sample of periodic variables.

\subsection{CLEAN periodogram}

The Scargle periodogram technique makes no attempt to account for the observational window function $W(v)$, i.e., some of the peaks in the Scargle periodogram are result of the data sampling. This effect is called aliasing and even the highest peak could be an artifact. The CLEAN periodogram technique by Roberts et al. (1987) tries to overcome this shortcoming of the Scargle periodogram. For a detailed description we refer the reader to Roberts et al. (1987) or Bailer-Jones \& Mundt (2001).

\section{Results}

For all the candidate cluster member stars that turned out from our analysis to be periodic variable, we list the target ID, RA, and Dec coordinates (J2000.0), extinction-corrected $V$ magnitude $V_{0}$ and colour $(B-V)_{0}$ in the online Table 1 . The results of our Fourier analysis are given in the online Table 2 where we list the star's ID; the period detected by Scargle $\left(P_{\text {Scargle }}\right)$ and by CLEAN $\left(P_{\text {CLEAN }}\right)$ algorithms; the normalised peak power $\left(P_{\mathrm{N}}\right)$; the adopted rotation period $(P)$, i.e., the period among $P_{\text {Scargle }}$ and $P_{\text {CLEAN }}$ giving the less scattered phased light curve, and its uncertainty as computed following Horne \& Baliunas (1986); the average $V$ magnitude $(\langle V\rangle)$; the light curve standard deviation $\left(\sigma_{\text {TOT }}\right)$; the achieved photometric accuracy $(\sigma)$; and the light curve amplitude $(\Delta V)$. The amplitude of the light curve was computed by making the difference between the median values of the upper and lower $15 \%$ of magnitudes of the light curve (see, e.g., Herbst et al. 2002). That allows us to prevent overestimation of the amplitude due to possible outliers. We also list the total number of observations, a note about either the membership or its ID for already known variables.

In Fig. 5, we display a selection of the most common results obtained with our period search analysis. In the panels from left to right, we plot the data time series ( $V$-band data vs. MJD). Different colours are used to distinguish three different datasets collected at different time intervals of the observing run. This helps to disentangle the scatter in the data arising from three different sets of observing runs. In fact, we noticed that a number of stars undergo changes in the light curve shape and amplitude on a timescale shorter than the whole time interval of 
observations (about 30 days). In the second panel from the left, we plot the light curve phased with the period reported in the label along with its uncertainty. We also report the data accuracy $(\sigma)$ and the light curve amplitude $(\Delta V)$. In the third panel, we plot the Scargle periodogram where the horizontal solid line represents the power level corresponding to the $99 \%$ confidence level. We note that in the 0.4-0.5 and 0.9-1.2 day range such level is set to a much higher power $\left(P_{\mathrm{N}}=31.64\right)$. The dashedvertical line indicates the selected periodicity. The right-most panel displays the power spectrum from CLEAN analysis.

We have cases, such as for star 02318 , in which only one period has a confidence level larger than $99 \%$, which is independently confirmed by the CLEAN analysis. There are cases, such as for star 02667, where two periods are detected. However, the shorter one is disregarded since it does not reach the power level of $P_{\mathrm{N}}=31.64$, which we set for the 0.9-1.2 day range. There are other cases, such as for star 03040, where two periods are detected with confidence level larger than 99\%. However, only one is confirmed by the CLEAN analysis. Finally, there are cases, such as for star 06040, where the period is detected in the 0.9-1.2 day range and has a power level larger that the one set for this period range. For 10 stars, two different periodicities of comparable power were detected by both Scargle and CLEAN periodograms. In these cases, we considered the period with higher power and which produces the less scattered phased light curve to be more reliable, and marked the second period to be an alias. In most cases the second periodicity represents a beat period $(B)$, which is related to the rotation period $(P)$ by the following relation:

$\frac{1}{B}=\frac{1}{P} \pm 1$

It can be identified to be an alias of the rotation period, since it produces a larger scatter in the phased light curve. We could detect periodicities with a high confidence level $(>99 \%)$ in a total of 135 stars.

We found 135 periodic variables, 16 of which were previously discovered by Kiss et al. (2001) and Kang et al. (2007). The total number of periodic cluster members we discovered is 106, which is the final sample analysed in the following sections.

In the online Figs. 11-13, we plot magnitudes time series, phased light curves and power spectra of the known variable $V 3-V 7$, discovered by Kiss et al. (2001), and $K V 3-K V 17$, discovered by Kang et al. (2007). We detected no evidence of eclipses for the variables $V 1$ and $V 2$, confirming the results of Kang et al. (2007), whereas the variables $K V 1$ and $K V 2$ got saturated in our long-exposures analysis. For 14 out of 20 remaining known periodic variables, our present analysis could confirm the previously-determined periods. For 6 out of $8 \delta$ Scuti stars we could detect the primary frequency. Six of of 20 known variable stars did not pass our period detection criteria, i.e., periodicity was detected with a low confidence level. For all the known contact eclipsing binaries our analysis detected the highest peak with greater than $99 \%$ confidence level at exactly half the orbital period. In fact, our Fourier analysis is better suited for single-peaked light variations, which are fitted by a single sinusoid function. In the present case, since the known contact eclipsing binaries have two minima of comparable depth, they have been all fitted by a single-peaked variation of half the orbital period. The very good agreement between the periods we detected and the previously-known periods for the common periodic variables makes us confident of the reliability of the periodicity measured for all the stars in our sample.

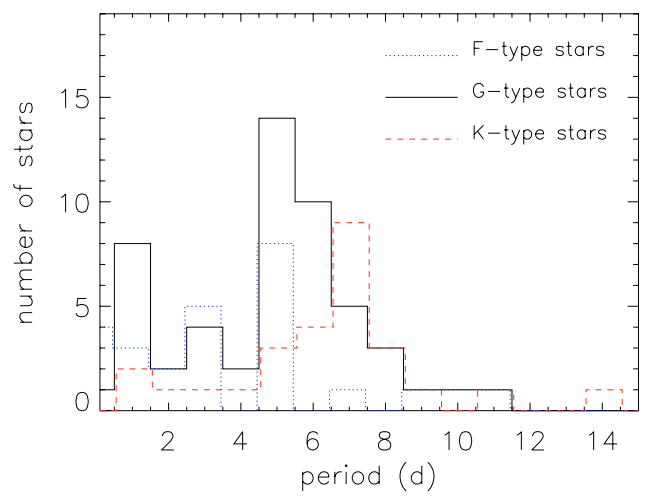

Fig. 6. Distributions of periods of the NGC 2099 candidate cluster members.

\section{Discussion}

\subsection{F-type stars}

As mentioned in Sect. 1, a search for variability among F-type stars in the NGC 2099 cluster was the prime motivation of the previous analysis carried out by Kang et al. (2007). That analysis allowed the discovery of a total of 24 periodic variable stars, including the 7 variables already identified by Kiss et al. (2001). Out of 17 newly-discovered variables by Kang et al. (2007), 9 variables are identified as $\delta$ Scuti-type pulsating stars, 7 are found to be contact eclipsing binaries, and one is a peculiar variable star. Although the present paper is focused on G- and K-type variable stars, we nonetheless also applied our Fourier analysis techniques, briefly described in Sect. 3, to the sub-sample of F-type candidate cluster members. Our new analysis has allowed us to discover 26 new periodic variables among candidate cluster members. Their light curves along with their Scargle and CLEAN periodograms, are shown in the online Figs. 14-16. The list of periodic F-type stars is given in Table 2 with indications of the variable stars already discovered in previous studies (Kiss et al. 2001; Kang et al. 2007).

The periodic variation of star light in F-type stars most likely arises from pulsations. About $70 \%$ of F-type variables have been found with periods most likely too long $\left(P^{\mathrm{d}}>2\right)$ to be attributed to pulsational modes (Fig. 6). One possibility is that these longperiod F-type variables may not be cluster members, but they are foreground field stars. Early G-type field stars may have been designated as cluster F-type variables due to reddening overcorrection. Consistent with this hypothesis, the $B-V$ colour of these stars is indeed at the boundary between inactive late F-type and active early-G stars. It is possible that further investigation of these stars may reveal that they are active G-type field stars.

\subsection{G- and K-type main-sequence stars}

The primary goal of the present study is to investigate the rotational and magnetic activity properties of late-type (G-K) members of NGC 2099. The Fourier analysis has allowed us to discover the periodicity in $52 \mathrm{G}$ - and $28 \mathrm{~K}$-type stars, whose masses cover approximately the $0.7 \lesssim M / M_{\odot} \lesssim 1.1$ range. The lower percentage of periodic $\mathrm{K}$ stars is largely ascribed to the lower photometric accuracy achieved for K stars with respect to $\mathrm{G}$ stars. The results of our Fourier analysis are summarised in the online Table 2, whereas the light curves, along with their Scargle and CLEAN periodograms, are plotted in the online Figs. 17-26. 

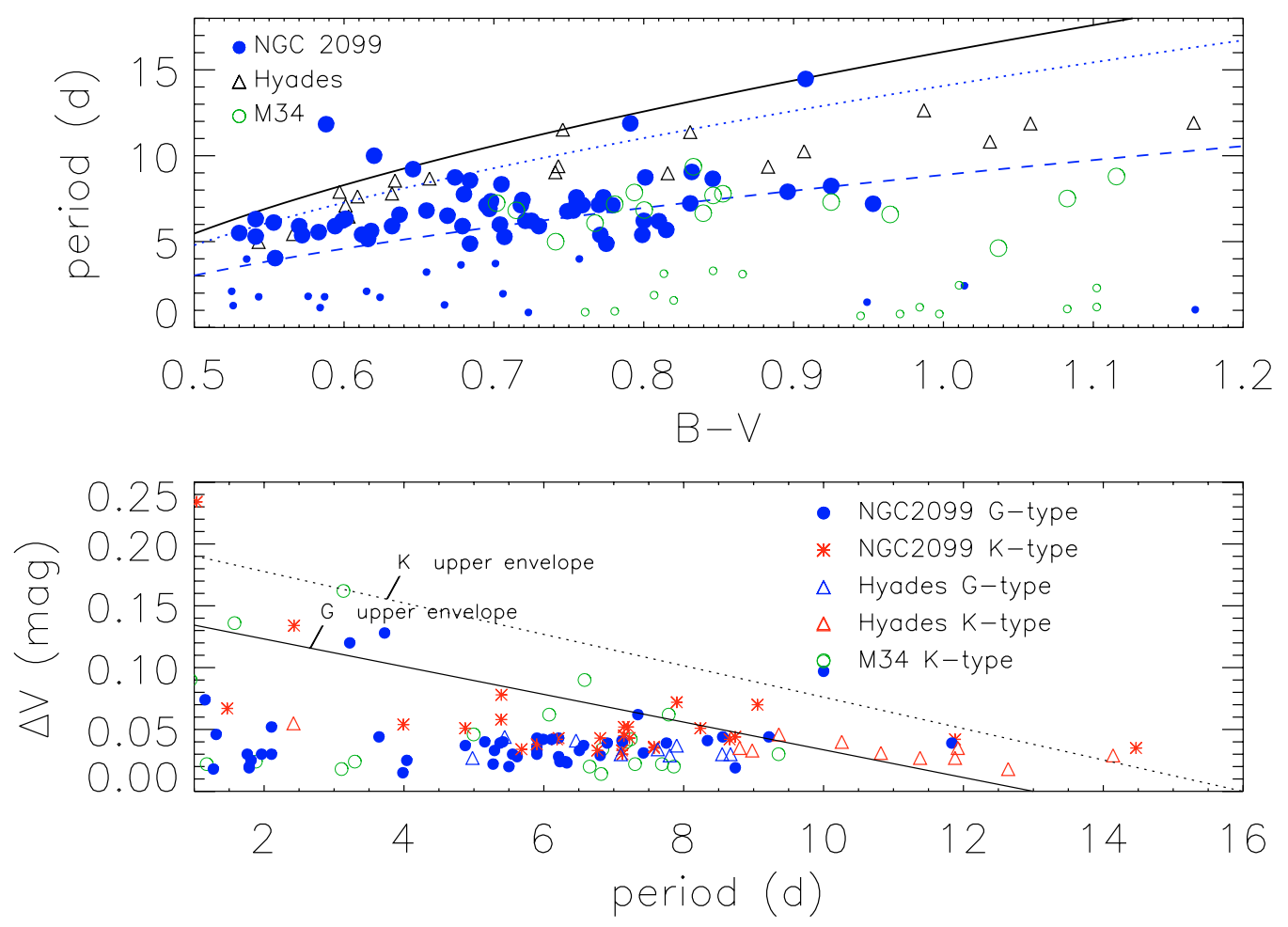

Fig. 7. Top panel: rotation period vs. $B-V$ colour for NGC 2099 (filled blue bullets), Hyades (open triangles), and M 34 members (open green bullets) with overplotted the family of age-parameterised curves from gyrochronology corresponding to ages of 600,500 and $200 \mathrm{Myr}$ (from top to bottom, respectively). Fast rotators with $P^{\mathrm{d}}<4$ among NGC 2099 and M 34 members are plotted with smaller symbols. Bottom panel: light curve amplitude $(\Delta V)$ vs. rotation period for NGC $2099 \mathrm{G}$-type (blue bullets) and K-type (red asterisks) members, G-type and K-type Hyades members (open blue and red triangles, respectively), and M 34 members (green open bullets). The upper limits of the $\Delta V-P$ relation for dwarf G (solid line) and K stars (dotted line) from Messina et al. (2003) are overplotted.

For these late-type stars the detected periodicity most likely represents the stellar rotation period. It is believed that in latetype stars the observed variability arises from nonuniformlydistributed, cool-spotted regions on the stellar photosphere, which are carried in and out of view by the star's rotation. In Fig. 6, we plot the distribution of rotation periods for G- and $\mathrm{K}$-type candidate cluster members. We note that our sample of $52 \mathrm{G}$ stars does not include the $10 \mathrm{~W}$ UMa-type eclipsing binaries, whose rotation period is altered by tidal synchronization and subjected to a different magnetic braking than in single stars. A Kolmogorov-Smirnov test reveals that $\mathrm{G}$ and $\mathrm{K}$ stars have different distributions, having K-type stars on average longer rotation periods than G-type stars. The distribution of G stars is peaked at a median period of $P=5.9$ days whereas the distribution of $\mathrm{K}$ stars at a median period of $P=7.1$ days.

In Fig. 6, we see that a secondary peak exists of fast rotating stars $\left(P^{\mathrm{d}}<3\right)$, consisting of about $20 \%$ of G stars. Such fast rotators are usually found in young open clusters but not expected in a cluster with an age of about $500 \mathrm{Myr}$ or older, which is the case of NGC 2099 (Barnes 2003, 2007). There are several possibilities for the presence of these fast rotators. Since the cluster is relatively far away $(1.4 \mathrm{kpc})$ and its galactic latitude is just 3 degrees, it may be contaminated by very young WTTS, which are usually fast rotators. Alternatively, some of these fast rotators may be cluster members, but may belong to close binaries of BY Dra, RS CVn, W UMa or FK Com types, which are rapidly rotating due to tidal interaction and synchronisation. A third possibility may be the false detections of the periods. For example, the presence of two activity centers in the stellar photosphere at the epochs of observations, about $180^{\circ}$ away in longitude from each other, can produce a light modulation with half of the true rotation period. In these cases, consecutive observation seasons are needed to detect the true rotation period (Padmakar Parihar et al. 2008). Identification of single fast rotating cluster members at an age of about $500 \mathrm{Myr}$ would be, indeed, very interesting and challenging to models of evolution of stellar angular momentum.

Our study of NGC 2099 is aimed at filling the gap in the description of the angular momentum and magnetic activity evolution in the age range from about 200 to $600 \mathrm{Myr}$. In the top panel of Fig. 7, we plot the rotation periods of the NGC 2099 $\mathrm{G}$ and $\mathrm{K}$ stars against their reddening corrected $B-V$ colour. We also include similar data of the slightly older ( $\sim 625 \mathrm{Myr}$; Perryman et al. 1998) Hyades open cluster from Radick et al. (1995) and of the younger ( 200 Myr; Irwin et al. 2006) M 34 cluster for a comparison. Such a comparison is restricted to the $0.7 \lesssim M / M_{\odot} \lesssim 1.1$ mass range, which roughly corresponds to the $0.52<(B-V)<1.0$ colour range within which most of the NGC 2099 candidate members fall. The $(V-I)$ colours of M 34 members were converted into $B-V$ colours by interpolating between colours of dwarf standard stars as tabulated in Cox (2000). Among the NGC 2099 and M 34 members, we identify a longer-period branch $\left(P^{\mathrm{d}}>4\right)$, which is represented by filled and open bullets in the top panel of Fig. 7. This branch is also present among Hyades stars (open triangles), and a short-period branch $\left(P^{\mathrm{d}}<4\right)$ which has no counterpart among Hyades stars.

Within the longer-period branch, we have computed the median period of G stars $(0.52<B-V \leq 0.74)$ in NGC 2099 and Hyades clusters, obtaining the following values: $P_{\text {Hyades }}^{\mathrm{d}}=7.6$ and $P_{\text {NGC } 2099}^{\mathrm{d}}=5.4$. Unfortunately, only three stars in M 34 fall within this colour range to compute a median value. The 
same was done for early $\mathrm{K}$ stars $(0.74<B-V \leq 1.0)$ in all three clusters, obtaining the following values: $P^{\mathrm{d}}$ Hyades $=10.3$, $P^{\mathrm{d}}{ }_{\text {NGC } 2099}=7.1$, and $P^{\mathrm{d}}{ }_{\mathrm{M} 34}=7.2$. From these median values we infer that $\mathrm{G}$ stars have undergone a significant spin down in the age range from $\sim 500$ to $\sim 600 \mathrm{Myr}$, as expected from magnetic breaking. The unexpected result is that $\mathrm{K}$ stars have kept their rotation period almost constant in the age range from $\sim 200$ to $\sim 500 \mathrm{Myr}$, and then they have undergone a significant spin down only in the following $100 \mathrm{Myr}$, i.e., until the Hyades age. Unfortunately, we do not have enough data for M 34 to check whether the rotation period of $\mathrm{G}$ stars has remained constant over the age range from $\sim 200$ to $\sim 500 \mathrm{Myr}$, or not. The difference in the rotation distribution of NGC 2099 stars with respect to Hyades stars is what is expected from the theory of angular momentum evolution, where the braking mechanism works more efficiently in K-type than in G-type stars. What is very different from theoretical expectations is that early K-type stars in NGC 2099 show the same rotation distribution as in M 34, that is they seem to have undergone no braking during the course of about $\sim 300$ Myr. We have also analysed our findings in the framework of the gyrochronology proposed by Barnes (2003, 2007). In Fig. 7, we plot the age-parameterized family of theoretical curves corresponding to nominal ages of the three clusters, that is, 625 Myr for Hyades (Perryman et al. 1998); 500 Myr for NGC 2099 (Hartman et al. 2008a); and 200 Myr for M 34 (Meynet et al. 1993), which are plotted as solid, dotted, and dashed lines, respectively. Whereas G stars in NGC 2099 seem to be in the age range between $\sim 200$ and $\sim 600 \mathrm{Myr}$, the early K stars are well fitted by the 200-Myr isochronal line, i.e., they appear to be rotationally unevolved from the age of M 34 ( 200 Myr).

Our measurements of rotation periods are very important since there is no determined period distribution at the age of about 500 Myr. In the most recent work on this subject (Irwin et al. 2008), the distribution of rotation periods in the 200-600 Myr range was interpolated. We have added an additional point to better constrain evolution models. On the other hand, our finding raises the question of why magnetic braking, at least in G8-K5 stars, has been ineffective in the age range from about 200 to 500 Myr. Presently, we are not in the position to give any reasonable explanation; however, once the rotation period distributions for the other target clusters of RACE-OC will be determined, we will be able to carry out a more accurate and robust comparison between observation and theory.

In the bottom panel of Fig. 7, we plot the light curve amplitudes vs. rotation period for the NGC 2099, Hyades, and M 34 clusters. As investigated by Messina et al. (2001, 2003), the maximum amplitude of light curve monotonically decreases with rotation period following power laws with different exponent in different mass ranges. Here, we overplot the fits from the cited works. We see that the amplitudes of periodic variables of NGC 2099 and M 34 are always smaller than the maximum expected amplitude. One explanation of these low amplitudes is that it is very unlikely to find a star at its maximum light curve amplitude from only one observing season. One needs 10-20 light curves collected over years to obtain a maximum amplitude for spotted variables (see, e.g., Messina et al. 2003; Messina 2008). But, at the same time, we argue that we have 31 variables stars within, e.g., the $P=5-7$ days rotation interval and over this period range, on a statistical basis at least, few stars are expected to show their maximum light curve amplitude, which we never find. Therefore, we suspect that there should be some other age-dependent quantity that controls the level of activity, other than rotation and mass, and makes older stars less

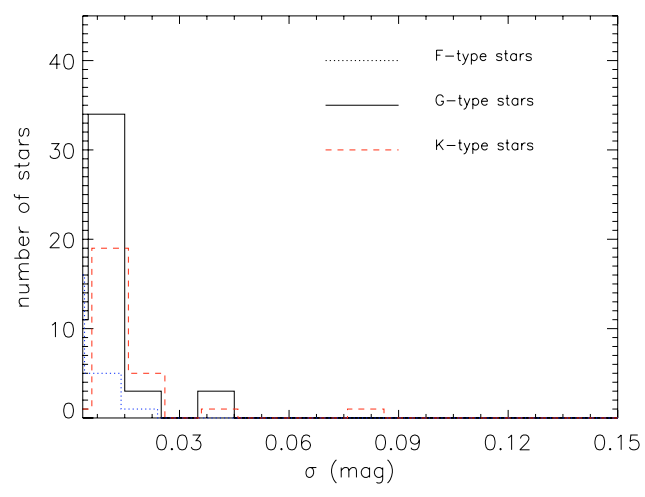

Fig. 8. Distributions of standard deviations of $V$-band light curves of periodic variables found in the open cluster NGC 2099.

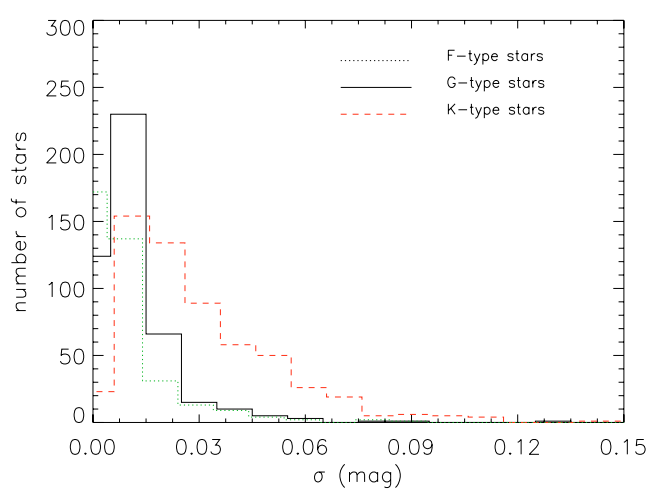

Fig. 9. Distributions of standard deviations of $V$-band light curves of stars identified as a non-periodic cluster variables.

active than younger. A similar suspect was already raised by Messina et al. (2001), who found evidence that, for a fixed mass and rotation period, the level of starspot activity increases from the zero-age main sequence up to the Pleiades age ( $\sim 130 \mathrm{Myr})$ and then it decreases with age. At an age of $200 \mathrm{Myr}$, the amplitude of K stars is significantly decreased with respect to K-type Pleiades stars, as shown in Fig. 16 of the paper by Irwin et al. (2006). Again, the younger G8-K5 stars in M 34 show a median light curve amplitude, which is a tracer of the level of photospheric magnetic activity, that is larger than the older NGC 2099 stars, although the mass range as well as the rotation period distribution are the same.

\subsection{Non-periodic variables}

By analysing the sample of $\mathrm{G}$ and $\mathrm{K}$ periodic stars, we find that the average ratio between the $V$-band light curve amplitude and its standard deviation is $2.7 \pm 0.1$, which must be considered valid for a type of variability arising from cool starspots. The expected maximum light curve amplitude of $\mathrm{G}$ and $\mathrm{K}$ stars is $\Delta V \simeq$ 0.15 and $\Delta V \simeq 0.20 \mathrm{mag}$, respectively (see Fig. 7). Therefore, stars having an observational accuracy better than $\sigma \simeq 0.07 \mathrm{mag}$ $(\simeq 0.20 / 2.7)$, which correspond to stars brighter than $V \simeq 20$ (Fig. 3), can be selected to search for periodic variability. Indeed, such accuracy allows us to detect with $3 \sigma$ confidence level, a variability of amplitude of $\simeq 0.20 \mathrm{mag}$, which is the maximum expected light curve amplitude for K-type stars.

Our Fourier analysis could not determine any significant periodicity for 1746 cluster members in the $13.0<V<20.0$ mag range. Nonetheless, some information on their nature of variability and membership can be derived from the analysis of 

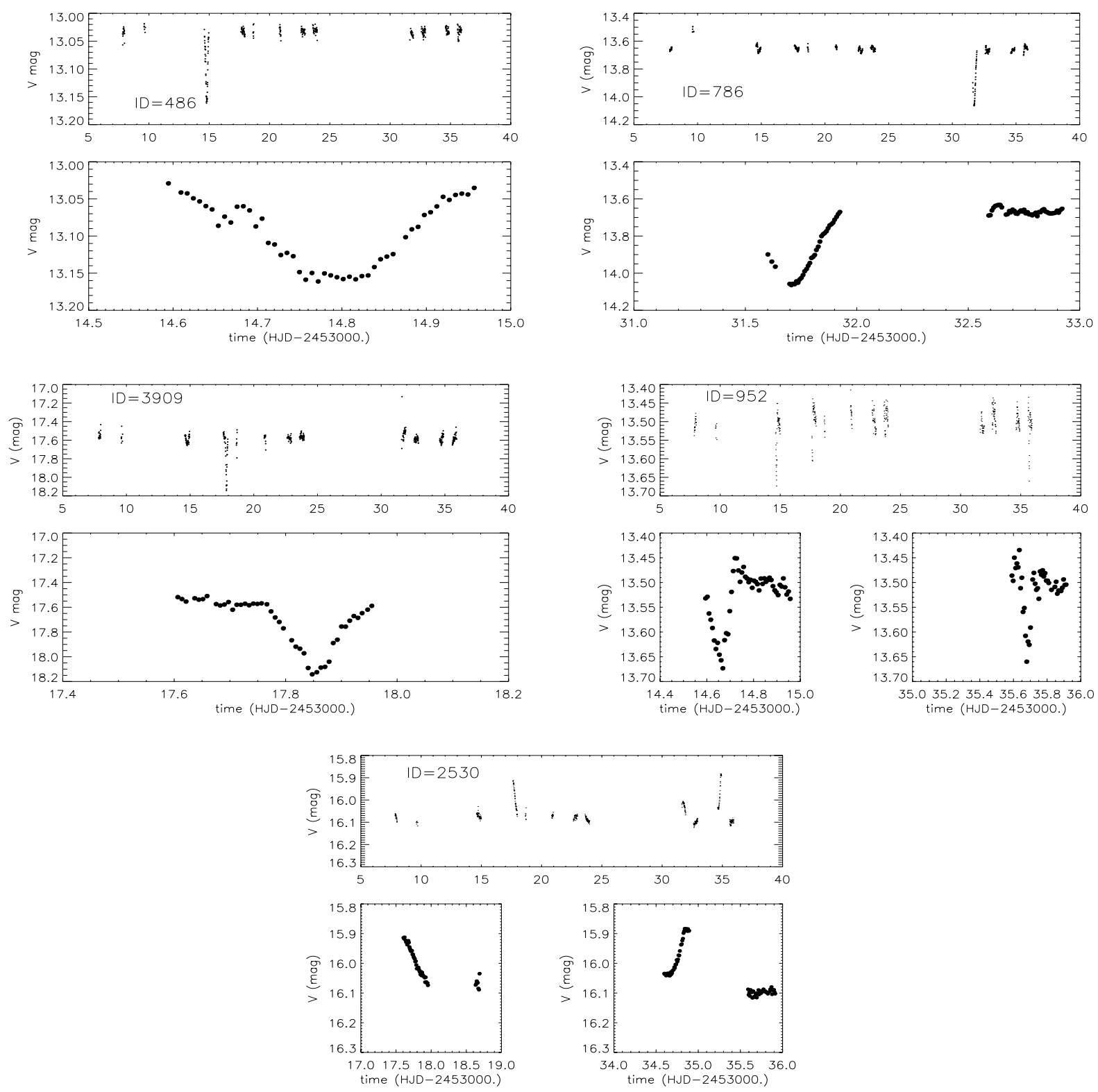

Fig. 10. Complete $V$-band magnitude time series (upper panels) of five interesting stars among newly-discovered cluster variables. Lower panels give an enlarged view of the most interesting nights. Stars ID $=486$, ID $=786$, ID $=3909$, and ID $=952$ are likely detached eclipsing binaries. ID $=2530$ shows outburst-like brightening up to $0.15 \mathrm{mag}$.

their standard deviations. In Fig. 8, we plot the $\sigma$ distribution of $V$-band light curves of periodic cluster variables. The median values of the plotted distributions are 0.013 and 0.016 mag for $\mathrm{G}$ and K-type stars, respectively, which is consistent with the behaviour shown by spotted stars. Although $\mathrm{K}$ stars on average rotate more slowly than $\mathrm{G}$ stars, they display larger photometric variability having deeper convection zones, more efficient dynamos, and consequently, a larger activity level. In Fig. 9, we plot the $\sigma$ distribution of all nonperiodic cluster variables. The median values of $\sigma$ for $\mathrm{G}$ and K-type stars are found to 0.013 and $0.028 \mathrm{mag}$, respectively. The distributions of $\sigma$ of periodic and nonperiodic $\mathrm{G}$ stars are similar, whereas for K-type stars the nonperiodic stars have larger $\sigma$ and a tail toward larger values, but they never exceed the 0.07 mag limit expected for active spotted stars. The discrepancy in the distribution between periodic and nonperiodic K-type stars may arise from contamination of noncluster stars, which we can guess are still spotted stars, but more active, and, therefore, younger and/or faster rotating.

\subsection{Individual stars}

Our analysis has allowed us to discover 5 variables, which certainly deserve further observations to characterise their nature and origin of variability. Four out of five variables are likely detached eclipsing binaries (Fig. 10): ID $=486$, ID $=786$, ID = 3909 , and ID $=952$. For ID $=486(V \simeq 12.4, B-V \simeq 0.12)$, we observed the full eclipse on HJD $=2453014$ of $\Delta V \simeq 0.13$ mag, of about $8.9 \mathrm{~h}$ of duration and with the minimum occurring at $\mathrm{HJD}_{\min }=2453014.81$. For ID $=786(V \simeq 13, B-V \simeq 0.30)$, we observed one incomplete eclipse on HJD $=2453031$ of $\Delta V \simeq$ $0.53 \mathrm{mag}$, of about $12 \mathrm{~h}$ of duration and with the minimum occurring at $\mathrm{HJD}_{\min }=2453031.70$. For $\mathrm{ID}=3909(V \simeq 16.9$, $B-V \simeq 0.54)$, we observed one almost complete eclipse on $\mathrm{HJD}=2453017$ of $\Delta V \simeq 0.65 \mathrm{mag}$, of about $3.9 \mathrm{~h}$ of duration and with the minimum occurring at $\mathrm{HJD}_{\min }=2453017.86$. For $\mathrm{ID}=952(V \simeq 12.8, B-V \simeq 1$. $)$, although the data are quite noisy, we observed two almost complete eclipses on HJD $=2453014$ 
and 2453035 of similar $\Delta V \simeq 0.18 \mathrm{mag}$, of about $2.5 \mathrm{~h}$ of duration and with the minima occurring at $\mathrm{HJD}_{\min }=2453014.67$ and 2453035.68 . These detached eclipsing binary candidates are very important since three of them are candidate cluster members, according to our selection criterion. Therefore, they allow us to make an accurate determination of mass and radius and, very importantly, a more constrained comparison of various stellar evolutionary models. Finally, ID $=2530(V \simeq 15.4$, $B-V \simeq 0.45$ ) shows two outburst-like events on different nights HJD $=2453017$ and 2453034, which last several hours and during which it gets brighter up to $0.15 \mathrm{mag}$. The origin is quite intriguing because the shape of the light curve at the brightening phase as well the star's spectral type are not consistent with a flare-like event.

\section{Conclusions}

In the present paper, we have presented the first results on variability and rotation of the G- and K-type members of the 500-Myr old open cluster NGC 2099, which has been studied as a part of the RACE-OC project. The photometric accuracy achieved in our observations has allowed us to explore presence of variability from F to middle K-type stars. Among 1746 candidate cluster members, we identified 122 periodic variables (16 of which were already known periodic variables). Whereas the periodicity of F-type stars likely arises from the presence of pulsations, for $80 \mathrm{G}$ - and K-type stars the detected periodicity arises from starspot activity and represents the stars' rotation period. We have, for the first time, determined the distribution of rotation period for these active cluster variables and found that rotation period of $\mathrm{G}$ and $\mathrm{K}$ stars have median value of $P=5.9$ and $P=7.1$ days, respectively. This mass-dependent difference tells us that, although K-type stars have reached the Zero Age Main Sequence later than G-type stars and, consequently, have started experiencing the rotation magnetic braking later, the braking has been more effective for $\mathrm{K}$ - than for G-type stars. The newly-determined rotation periods of the NGC 2099 candidate members are very important since they allow us to partly fill the gap in the empirical description of the angular momentum evolution in the age interval from 200 to 600 Myr. A comparison with rotation data from the younger M 34 ( $200 \mathrm{Myr})$ and the older Hyades clusters ( 625 Myr) shows that the NGC 2099 members rotate faster than the older Hyades stars, with a clear mass dependency, as expected from angular momentum evolution models. The interesting result is that G8-K5 members of NGC 2099 have the same rotation period distribution of the younger G8-K5 members of M 34. These stars seem to have experienced no braking in the age range from 200 to 500 Myr. Finally, the new light curve amplitude data of NGC 2099 give some further support to the suspicion that the level of magnetic activity, in the photosphere at least, has some other not yet identified age dependence, which makes stars older than Pleiades less and less active, independent from mass and rotation.

Acknowledgements. This work was supported by the Italian Ministero della Ricerca (MUR), Italian Ministero degli Affari Esteri (MAE) and Indian Department of Science and Technology (DST). The extensive use of the SIMBAD and ADS databases operated by the CDS center, Strasbourg, France, is gratefully acknowledged. The work of S.-C. R. was supported in part by KOSEF through the Astrophysical Research Center for the Structure and Evolution of the Cosmos (ARCSEC).

\section{References}

Bailer-Jones, C. A. L., \& Mundt, R. 2001, A\&A, 367, 218

Barnes, S. 2003, ApJ, 586, 464

Barnes, S. 2007, ApJ, 669, 1167

Bouvier, J., Forestini, M., \& Allain, S. 1997, A\&A, 326, 1023

Cox, A. N. 2000, in Allen's Astrophysical Quantities, 4th edn. (Springer, AIP Press)

Guinan, E. F., McCook, G. P., DeWarf, L. E., et al. 2003, BAAS, 35, 766

Hartman, J. D., Gaudi, B. S., Holman, M. J., et al. 2008a, ApJ, 675, 1233

Hartman, J. D., Gaudi, B. S., Holman, M. J., et al. 2008b, ApJ, 675, 1254

Herbst, W., \& Wittenmyer, R. 1996, BAAS, 28, 1338

Herbst, W., \& Mundt, R. 2005, ApJ, 633, 967

Herbst, W., Bailer-Jones, C. A. L., Mundt, R., Meisenheimer, K., \& Wackermann, R. 2002, A\&A, 396, 513

Hodgkin, S. T., Irwin, J. M., Aigrain, S., et al. 2006, AN, 327, 9

Holzwarth, V., \& Jardine, M. 2007, A\&A, 463, 11

Horne, J. H., \& Baliunas, S. L. 1986, ApJ, 302, 757

Ivanova, N., \& Taam, R. E. 2003, ApJ, 599, 516

Irwin, J., Aigrain, S., Hodgkin, S., et al. 2006, MNRAS, 370, 954

Irwin, J., Hodgkin, S., Aigrain, S., et al. 2008, MNRAS, 383, 1588

Kang, Y. B., Kim, S.-L., Rey, S.-C., et al. 2007, PASP, 119, 239

Kawaler, S. D. 1988, ApJ, 333, 236

Kiss, L. L., Szabò, Gy. M., Sziládi, K., et al. 2001, A\&A, 376, 561

Krishnamurthi, A., Pinsonneault, M. H., Barnes, S., \& Sofia, S. 1997, ApJ, 480, 303

MacGregor, K. B., \& Brenner, M. 1991, ApJ, 376, 204

Mermilliod, J.-C., Huestamendia, G., del Rio, G., \& Mayor, M. 1996, A\&A, 307, 80

Messina, S. 2007, Mem. Soc. Astron. It., 78, 628

Messina, S. 2008, A\&A, 480, 495

Messina, S., Rodonò, M., \& Guinan, E. F. 2001, A\&A, 366, 215

Messina, S., Pizzolato, N., Guinan, E. F., \& Rodonó, M. 2003, A\&A, 410, 671

Meynet, G., Mermilliod, J.-C., \& Maeder, A. 1993, A\&AS, 98, 477

Nilakshi, \& Sagar, R. 2002, A\&A, 381, 65

Parihar Padmakar, S., Messina, S., Distefano, E., et al. 2008, in preparation

Perryman, M. A. C., Brown, A. G. A., Lebreton, Y., et al. 1998, A\&A, 331, 81

Press, W. H., Teukolsky, S. A., Vetterling, W. T., \& Flannery, B. P. 1992, in Numerical recipes in FORTRAN, 2nd edn. (Cambridge: University Press)

Radick, R. R., Lockwood, G. W., Skiff, B. A., \& Thompson, D. T. 1995, ApJ, 452, 332

Rebull, L. M., Wolff, S. C., \& Strom, S. E. 2004, AJ, 127, 1029

Roberts, D. H., Lehar, J., \& Dreher, J. W. 1987, AJ, 93, 978

Roze, M. B., \& Hintz, E. G. 2007, AJ, 134, 2067

Rodonò, M., Messina, S., Lanza, A. F., Cutispoto, G., \& Teriaca, L. 2000, A\&A, 358,624

Scargle, J. D. 1982, ApJ, 263, 835

Stassun, K. G., Mathieu, R. D., Mazeh, T., \& Vrba, F. 1999, AJ, 117, 2941

Sills, A., Pinsonneault, M. H., \& Terndrup, D. M. 2000, ApJ, 534, 335

von Braun, K., Lee, B. L., Seager, S., et al. 2005, PASP, 117, 141 
Table 1. ID of periodic variables, RA, and Dec coordinates (J2000), average $V$ magnitude $\left(V_{\text {mean }}\right)_{0}$ corrected for extinction and de-reddened $(B-V)_{0}$ color.

\begin{tabular}{|c|c|c|c|c|}
\hline ID & $\begin{array}{c}\text { RA } \\
\text { (hh:mm:ss) }\end{array}$ & $\begin{array}{c}\text { Dec } \\
\text { (dd:mm:ss) }\end{array}$ & $\begin{array}{c}\left(V_{\text {mean }}\right)_{0} \\
(\mathrm{mag})\end{array}$ & $\begin{array}{c}(B-V)_{0} \\
(\mathrm{mag})\end{array}$ \\
\hline 59 & $05: 52: 11.286$ & $+32: 34: 11.06$ & 13.281 & 0.209 \\
\hline 80 & 05:52:21.216 & $+32: 34: 16.91$ & 14.451 & 0.492 \\
\hline 96 & 05:52:27.800 & $+32: 33: 38.69$ & 13.941 & 0.369 \\
\hline 263 & 05:52:12.768 & $+32: 28: 41.15$ & 14.301 & 0.426 \\
\hline 267 & 05:52:08.778 & $+32: 28: 33.38$ & 13.801 & 0.350 \\
\hline 290 & 05:51:59.524 & $+32: 34: 16.49$ & 12.911 & 0.153 \\
\hline 295 & 05:52:03.702 & $+32: 35: 14.21$ & 14.741 & 0.317 \\
\hline 320 & 05:52:14.955 & $+32: 37: 06.04$ & 14.331 & 0.276 \\
\hline 352 & 05:52:40.942 & $+32: 32: 01.70$ & 13.651 & 0.325 \\
\hline 370 & 05:52:33.520 & $+32: 28: 59.89$ & 13.831 & 0.363 \\
\hline 385 & 05:52:08.058 & $+32: 27: 34.82$ & 13.961 & 0.502 \\
\hline 414 & 05:51:47.281 & $+32: 32: 11.84$ & 13.611 & 0.249 \\
\hline 427 & 05:51:52.715 & $+32: 35: 17.25$ & 14.611 & 0.538 \\
\hline 441 & 05:52:00.816 & $+32: 36: 48.67$ & 13.881 & 0.431 \\
\hline 483 & 05:52:50.944 & $+32: 34: 48.84$ & 14.871 & 0.620 \\
\hline 486 & 05:52:45.611 & $+32: 33: 38.36$ & 12.361 & 0.118 \\
\hline 491 & $05: 52: 46.210$ & $+32: 32: 35.88$ & 14.471 & 0.433 \\
\hline 492 & 05:52:45.834 & $+32: 32: 20.36$ & 14.181 & 0.369 \\
\hline 493 & 05:52:46.479 & $+32: 32: 12.72$ & 14.881 & 0.479 \\
\hline 512 & 05:52:35.713 & $+32: 26: 38.04$ & 13.781 & 0.262 \\
\hline 548 & 05:51:47.557 & $+32: 28: 26.05$ & 14.651 & 0.345 \\
\hline 566 & 05:51:38.218 & $+32: 31: 47.94$ & 14.551 & 0.133 \\
\hline 570 & 05:51:40.520 & $+32: 32: 32.48$ & 13.811 & 0.419 \\
\hline 595 & 05:52:02.827 & $+32: 40: 09.13$ & 15.111 & 0.172 \\
\hline 643 & 05:52:53.438 & $+32: 29: 51.64$ & 13.831 & 0.310 \\
\hline 666 & 05:52:12.182 & $+32: 24: 34.15$ & 13.841 & 0.691 \\
\hline 761 & 05:53:01.748 & $+32: 28: 40.53$ & 12.851 & 0.135 \\
\hline 764 & 05:52:50.548 & $+32: 26: 27.94$ & 13.741 & 0.345 \\
\hline 786 & 05:52:00.961 & $+32: 22: 20.29$ & 13.001 & 0.283 \\
\hline 855 & 05:52:36.952 & $+32: 43: 18.78$ & 13.771 & 0.084 \\
\hline 857 & 05:52:43.801 & $+32: 43: 49.72$ & 13.981 & 0.528 \\
\hline 871 & 05:53:10.740 & $+32: 33: 47.22$ & 13.200 & 0.312 \\
\hline 916 & 05:51:30.381 & $+32: 25: 22.05$ & 15.111 & 0.404 \\
\hline 952 & 05:51:51.693 & $+32: 43: 49.99$ & 12.821 & 1.008 \\
\hline 969 & 05:53:06.085 & $+32: 41: 36.58$ & 14.511 & 0.351 \\
\hline 2112 & 05:51:56.717 & $+32: 35: 16.54$ & 15.981 & 0.687 \\
\hline 2257 & 05:52:26.549 & $+32: 40: 45.29$ & 15.351 & 0.549 \\
\hline 2312 & 05:52:18.846 & $+32: 33: 36.14$ & 16.041 & 0.654 \\
\hline 2318 & 05:52:22.711 & $+32: 32: 28.78$ & 14.821 & 0.580 \\
\hline 2321 & 05:52:18.530 & $+32: 31: 50.81$ & 16.521 & 0.799 \\
\hline 2330 & 05:52:13.920 & $+32: 33: 19.59$ & 16.321 & 0.640 \\
\hline 2385 & 05:52:27.231 & $+32: 34: 45.24$ & 15.501 & 0.334 \\
\hline 2395 & 05:52:31.316 & $+32: 32: 49.32$ & 16.511 & 0.765 \\
\hline 2408 & $05: 52: 24.266$ & $+32: 30: 32.11$ & 16.661 & 0.839 \\
\hline 2409 & 05:52:22.439 & $+32: 30: 30.90$ & 16.421 & 0.726 \\
\hline 2443 & 05:52:26.522 & $+32: 36: 23.97$ & 15.901 & 0.663 \\
\hline 2444 & 05:52:27.104 & $+32: 36: 10.71$ & 15.501 & 0.607 \\
\hline 2456 & 05:52:33.022 & $+32: 32: 41.79$ & 15.340 & 0.295 \\
\hline 2471 & 05:52:28.149 & $+32: 29: 55.11$ & 15.961 & 0.668 \\
\hline 2472 & 05:52:27.495 & $+32: 29: 41.03$ & 16.911 & 0.957 \\
\hline 2473 & $05: 52: 23.246$ & $+32: 29: 19.41$ & 16.451 & 0.747 \\
\hline 2485 & 05:52:01.813 & $+32: 32: 11.12$ & 15.031 & 0.469 \\
\hline 2506 & 05:52:19.780 & $+32: 37: 30.37$ & 16.901 & 0.692 \\
\hline 2509 & 05:52:20.404 & $+32: 38: 44.19$ & 16.391 & 0.916 \\
\hline 2530 & $05: 52: 31.672$ & $+32: 36: 32.97$ & 15.381 & 0.447 \\
\hline 2541 & 05:52:36.865 & $+32: 36: 53.36$ & 16.851 & 0.778 \\
\hline 2549 & 05:52:37.672 & $+32: 35: 17.70$ & 15.561 & 0.551 \\
\hline 2557 & 05:52:38.088 & $+32: 34: 16.43$ & 16.211 & 0.440 \\
\hline 2562 & 05:52:44.972 & $+32: 33: 51.22$ & 16.101 & 0.632 \\
\hline 2595 & 05:52:42.188 & $+32: 29: 38.50$ & 16.591 & 0.809 \\
\hline 2596 & 05:52:39.968 & $+32: 29: 22.42$ & 15.501 & 0.416 \\
\hline
\end{tabular}

Table 1. continued.

\begin{tabular}{|c|c|c|c|c|}
\hline ID & $\begin{array}{c}\text { RA } \\
\text { (hh:mm:ss) }\end{array}$ & $\begin{array}{c}\text { Dec } \\
\text { (dd:mm:ss) }\end{array}$ & $\begin{array}{c}\left(V_{\text {mean }}\right)_{0} \\
(\mathrm{mag})\end{array}$ & $\begin{array}{c}(B-V)_{0} \\
(\mathrm{mag})\end{array}$ \\
\hline 2597 & $05: 52: 39.476$ & $+32: 29: 00.09$ & 16.781 & 0.808 \\
\hline 2610 & $05: 52: 32.822$ & $+32: 30: 24.92$ & 16.151 & 0.688 \\
\hline 2618 & $05: 52: 33.751$ & $+32: 28: 06.57$ & 16.251 & 0.787 \\
\hline 2635 & $05: 52: 15.993$ & $+32: 28: 04.51$ & 15.631 & 0.591 \\
\hline 2639 & $05: 52: 14.653$ & $+32: 28: 45.35$ & 16.831 & 0.781 \\
\hline 2646 & $05: 52: 12.320$ & $+32: 27: 47.38$ & 17.131 & 0.961 \\
\hline 2650 & 05:52:08.712 & $+32: 28: 55.25$ & 15.541 & 0.850 \\
\hline 2665 & 05:51:58.835 & $+32: 29: 56.21$ & 16.371 & 0.727 \\
\hline 2667 & 05:52:00.312 & $+32: 30: 33.97$ & 15.141 & 0.505 \\
\hline 2676 & $05: 51: 58.275$ & $+32: 31: 15.56$ & 14.821 & 0.478 \\
\hline 2681 & $05: 51: 57.034$ & $+32: 31: 36.18$ & 15.591 & 0.626 \\
\hline 2687 & $05: 51: 51.187$ & $+32: 32: 36.17$ & 15.501 & 0.508 \\
\hline 2701 & $05: 51: 52.245$ & $+32: 34: 19.87$ & 14.711 & 0.489 \\
\hline 2703 & 05:51:55.647 & $+32: 34: 18.35$ & 15.421 & 0.682 \\
\hline 2728 & 05:52:03.061 & $+32: 38: 19.21$ & 15.751 & 0.645 \\
\hline 2734 & 05:52:09.443 & $+32: 37: 03.29$ & 16.411 & 0.763 \\
\hline 2742 & 05:52:17.219 & $+32: 37: 27.87$ & 15.101 & 0.507 \\
\hline 2743 & $05: 52: 18.470$ & $+32: 40: 22.54$ & 16.231 & 0.592 \\
\hline 2763 & $05: 52: 26.055$ & $+32: 40: 01.70$ & 16.211 & 0.675 \\
\hline 2832 & $05: 52: 53.511$ & $+32: 33: 01.48$ & 15.261 & 0.599 \\
\hline 2835 & 05:53:00.720 & $+32: 32: 06.26$ & 15.681 & 0.424 \\
\hline 2843 & $05: 52: 53.330$ & $+32: 31: 41.30$ & 16.361 & 0.692 \\
\hline 2847 & $05: 52: 55.483$ & $+32: 31: 04.34$ & 14.941 & 0.534 \\
\hline 2857 & $05: 52: 44.894$ & $+32: 30: 17.51$ & 15.372 & 0.480 \\
\hline 2859 & $05: 52: 46.090$ & $+32: 29: 36.08$ & 15.621 & 0.609 \\
\hline 2862 & $05: 52: 50.636$ & $+32: 29: 00.59$ & 15.061 & 1.261 \\
\hline 2874 & $05: 52: 53.400$ & $+32: 27: 14.14$ & 15.621 & 0.436 \\
\hline 2890 & $05: 52: 38.962$ & $+32: 28: 09.34$ & 16.951 & 0.933 \\
\hline 2895 & $05: 52: 38.385$ & $+32: 27: 19.72$ & 15.371 & 0.595 \\
\hline 2947 & $05: 52: 19.351$ & $+32: 26: 22.48$ & 15.151 & 0.543 \\
\hline 2948 & $05: 52: 22.001$ & $+32: 25: 15.94$ & 15.441 & 0.715 \\
\hline 2968 & $05: 52: 14.556$ & $+32: 23: 48.43$ & 16.341 & 0.729 \\
\hline 2971 & $05: 52: 12.781$ & $+32: 24: 02.70$ & 15.661 & 0.772 \\
\hline 2973 & $05: 52: 11.646$ & $+32: 25: 17.97$ & 16.056 & 0.149 \\
\hline 2974 & $05: 52: 12.321$ & $+32: 26: 10.25$ & 15.651 & 0.584 \\
\hline 2995 & 05:52:08.122 & $+32: 26: 40.53$ & 15.731 & 0.563 \\
\hline 2996 & 05:52:09.242 & $+32: 26: 41.95$ & 16.431 & 0.733 \\
\hline 2997 & 05:52:06.024 & $+32: 27: 08.38$ & 16.101 & 0.703 \\
\hline 3003 & 05:52:02.651 & $+32: 24: 54.32$ & 16.311 & 0.705 \\
\hline 3008 & 05:51:57.938 & $+32: 25: 06.26$ & 16.291 & 0.623 \\
\hline 3021 & $05: 52: 01.447$ & $+32: 26: 46.04$ & 15.021 & 0.628 \\
\hline 3030 & 05:51:48.881 & $+32: 26: 26.38$ & 16.561 & 0.714 \\
\hline 3031 & 05:51:50.810 & $+32: 25: 48.55$ & 15.161 & 0.475 \\
\hline 3040 & 05:51:49.605 & $+32: 28: 13.18$ & 15.051 & 0.477 \\
\hline 3082 & $05: 51: 43.681$ & $+32: 30: 40.53$ & 16.641 & 0.761 \\
\hline 3089 & $05: 51: 34.133$ & $+32: 30: 40.44$ & 15.081 & 0.511 \\
\hline 3090 & $05: 51: 32.827$ & $+32: 31: 45.45$ & 15.811 & 0.313 \\
\hline 3094 & 05:51:49.489 & $+32: 31: 38.83$ & 16.041 & 0.663 \\
\hline 3110 & $05: 51: 32.724$ & $+32: 32: 26.20$ & 15.541 & 0.533 \\
\hline 3146 & 05:51:50.368 & $+32: 35: 13.53$ & 15.131 & 0.477 \\
\hline 3157 & 05:51:47.902 & $+32: 36: 01.59$ & 15.531 & 0.578 \\
\hline 3208 & 05:51:58.335 & $+32: 39: 42.38$ & 16.291 & 0.731 \\
\hline 3209 & $05: 51: 58.156$ & $+32: 39: 46.40$ & 15.661 & 0.602 \\
\hline 3230 & 05:52:07.245 & $+32: 39: 14.15$ & 16.441 & 0.713 \\
\hline 3236 & $05: 52: 06.222$ & $+32: 41: 25.42$ & 16.261 & 0.854 \\
\hline 3245 & 05:52:08.640 & $+32: 41: 18.84$ & 16.591 & 0.779 \\
\hline 3246 & 05:52:11.343 & $+32: 41: 44.50$ & 16.652 & 0.524 \\
\hline 3247 & $05: 52: 10.953$ & $+32: 41: 19.94$ & 16.141 & 0.596 \\
\hline 3294 & 05:51:59.018 & $+32: 44: 03.92$ & 15.941 & 0.706 \\
\hline 3452 & $05: 52: 58.280$ & $+32: 38: 44.74$ & 14.521 & 0.708 \\
\hline 3512 & $05: 51: 31.962$ & $+32: 34: 21.12$ & 16.501 & 0.677 \\
\hline 3550 & 05:53:07.503 & $+32: 30: 58.67$ & 16.125 & 0.271 \\
\hline 3582 & 05:51:33.040 & $+32: 28: 45.77$ & 16.601 & 0.783 \\
\hline
\end{tabular}


Table 1. continued.

\begin{tabular}{ccccc}
\hline \hline ID & $\begin{array}{c}\text { RA } \\
\text { (hh:mm:ss) }\end{array}$ & $\begin{array}{c}\text { Dec } \\
\text { dd:mm:ss })\end{array}$ & $\begin{array}{c}\left(V_{\text {man }}\right)_{0} \\
(\mathrm{mag})\end{array}$ & $\begin{array}{c}(B-V)_{0} \\
(\mathrm{mag})\end{array}$ \\
\hline 3617 & $05: 51: 32.229$ & $+32: 27: 11.21$ & 15.941 & 0.549 \\
3638 & $05: 53: 09.357$ & $+32: 26: 23.33$ & 16.081 & 0.818 \\
3702 & $05: 52: 08.552$ & $+32: 22: 38.90$ & 16.291 & 0.767 \\
3712 & $05: 52: 32.702$ & $+32: 22: 57.12$ & 16.171 & 0.763 \\
3713 & $05: 52: 34.629$ & $+32: 22: 58.49$ & 16.241 & 0.686 \\
3714 & $05: 52: 36.626$ & $+32: 23: 01.65$ & 16.301 & 0.738 \\
3724 & $05: 53: 00.859$ & $+32: 24: 51.65$ & 15.411 & 0.604 \\
3725 & $05: 52: 58.565$ & $+32: 23: 26.78$ & 16.351 & 0.561 \\
3866 & $05: 52: 34.784$ & $+32: 28: 25.90$ & 15.281 & 0.624 \\
3909 & $05: 51: 56.038$ & $+32: 33: 25.03$ & 16.901 & 0.541 \\
4035 & $05: 52: 42.224$ & $+32: 27: 31.65$ & 15.121 & 0.562 \\
4035 & $05: 52: 42.224$ & $+32: 27: 31.65$ & 15.121 & 0.562 \\
4103 & $05: 51: 33.453$ & $+32: 39: 28.59$ & 16.281 & 0.807 \\
4115 & $05: 52: 08.437$ & $+32: 32: 15.76$ & 16.221 & -2.206 \\
4134 & $05: 52: 46.232$ & $+32: 25: 34.30$ & 16.691 & 0.823 \\
4181 & $05: 52: 39.365$ & $+32: 36: 29.83$ & 16.951 & 0.566 \\
4326 & $05: 52: 24.415$ & $+32: 24: 44.93$ & 17.561 & 1.268 \\
4402 & $05: 52: 47.663$ & $+32: 39: 33.20$ & 17.199 & 0.588 \\
4423 & $05: 51: 47.965$ & $+32: 36: 51.93$ & 17.281 & 0.904 \\
4481 & $05: 52: 48.453$ & $+32: 36: 42.43$ & 17.281 & 0.840 \\
4576 & $05: 53: 08.465$ & $+32: 33: 47.47$ & 18.001 & 0.755 \\
4654 & $05: 53: 03.640$ & $+32: 32: 12.83$ & 17.877 & 0.691 \\
4708 & $05: 52: 41.016$ & $+32: 24: 24.60$ & 17.359 & 0.859 \\
4822 & $05: 53: 04.053$ & $+32: 29: 41.74$ & 18.211 & 1.022 \\
5082 & $05: 52: 15.809$ & $+32: 43: 36.20$ & 15.821 & 0.712 \\
5893 & $05: 51: 34.735$ & $+32: 29: 06.72$ & 20.066 & 0.411 \\
5906 & $05: 52: 44.159$ & $+32: 28: 52.05$ & 19.749 & 0.646 \\
6040 & $05: 52: 49.442$ & $+32: 26: 17.52$ & 18.301 & 1.176 \\
6462 & $05: 52: 57.457$ & $+32: 41: 15.44$ & 17.221 & 0.709 \\
7941 & $05: 51: 29.856$ & $+32: 24: 19.35$ & 16.896 & 0.594 \\
\hline & & & &
\end{tabular}


Table 2. Star's ID, period detected by Scargle $\left(P_{\text {Scargle }}\right)$ and by CLEAN $\left(P_{\text {CLEAN }}\right)$, normalised peak power $\left(P_{\mathrm{N}}\right)$, adopted rotation period $(P)$ and its uncertainty, average $V$ magnitude $(\langle V\rangle)$, standard deviation $\left(\sigma_{\mathrm{TOT}}\right)$, achieved photometric accuracy, light curve amplitude $(\Delta V)$, number of observations, note about the membership ( $\mathrm{cm}$ is candidate member).

\begin{tabular}{|c|c|c|c|c|c|c|c|c|c|c|}
\hline ID & $\begin{array}{r}P_{\text {Scargle }} \\
\text { (d) }\end{array}$ & $\begin{array}{r}P_{\text {CLEAN }} \\
\text { (d) }\end{array}$ & $P_{\mathrm{N}}$ & $\begin{array}{r}P \pm \Delta P \\
(\mathrm{~d})\end{array}$ & $\begin{array}{l}V_{\text {mean }} \\
\text { (mag) }\end{array}$ & $\begin{array}{l}\sigma_{\mathrm{TOT}} \\
(\mathrm{mag})\end{array}$ & $\begin{array}{l}\text { Accuracy } \\
\text { (mag) }\end{array}$ & $\begin{array}{l}\Delta V \\
(\mathrm{mag})\end{array}$ & \# obs. & Note \\
\hline \multicolumn{11}{|c|}{ F-type periodic candidate members } \\
\hline 80 & 5.433 & 0.000 & 15.31 & $5.433 \pm 0.095$ & 15.13 & 0.0065 & 0.0031 & 0.0185 & 362 & $\mathrm{~cm}$ \\
\hline 96 & 2.170 & 0.000 & 15.80 & $2.170 \pm 0.014$ & 14.61 & 0.0118 & 0.0041 & 0.0338 & 362 & $\mathrm{~cm}$ \\
\hline 263 & 5.393 & 5.400 & 15.42 & $5.393 \pm 0.048$ & 14.98 & 0.0115 & 0.0033 & 0.0318 & 362 & $\mathrm{~cm}$ \\
\hline 290 & 0.339 & 0.337 & 16.49 & $0.339 \pm 0.003$ & 13.59 & 0.0066 & 0.0030 & 0.0176 & 358 & $\mathrm{~cm}$ \\
\hline 295 & 0.106 & 0.119 & 93.56 & $0.119 \pm 0.000$ & 15.42 & 0.0378 & 0.0100 & 0.1080 & 362 & $\delta$ Scuti (KV4) \\
\hline 320 & 5.354 & 0.840 & 15.44 & $5.354 \pm 0.003$ & 15.01 & 0.0077 & 0.0034 & 0.0210 & 362 & $\mathrm{~cm}$ \\
\hline 370 & 0.558 & 0.000 & 27.99 & $0.558 \pm 0.003$ & 14.51 & 0.0205 & 0.0039 & 0.0605 & 362 & $\mathrm{~cm}$ \\
\hline 385 & 1.911 & 1.899 & 35.21 & $1.911 \pm 0.005$ & 14.64 & 0.0222 & 0.0046 & 0.0565 & 362 & $\mathrm{~cm}$ \\
\hline 441 & 0.944 & 0.952 & 140.31 & $1.888 \pm 0.003$ & 14.56 & 0.0391 & 0.0100 & 0.1147 & 362 & EW (KV11) \\
\hline 491 & 1.732 & 1.732 & 21.00 & $1.732 \pm 0.063$ & 15.15 & 0.0078 & 0.0029 & 0.0215 & 362 & $\mathrm{~cm}$ \\
\hline 492 & 5.433 & 5.391 & 19.29 & $5.433 \pm 0.069$ & 14.86 & 0.0075 & 0.0030 & 0.0209 & 362 & $\mathrm{~cm}$ \\
\hline 493 & 9.533 & 10.000 & 17.96 & $9.533 \pm 0.279$ & 15.56 & 0.0069 & 0.0034 & 0.0203 & 362 & $\mathrm{~cm}$ \\
\hline 512 & 0.574 & 0.578 & 24.87 & $0.578 \pm 0.003$ & 14.46 & 0.0186 & 0.0028 & 0.0481 & 362 & $\mathrm{~cm}$ \\
\hline 548 & 0.556 & 0.602 & 16.02 & $0.556 \pm 0.003$ & 15.33 & 0.0103 & 0.0037 & 0.0283 & 362 & $\mathrm{~cm}$ \\
\hline 643 & 3.762 & 0.787 & 20.73 & $3.762 \pm 0.010$ & 14.51 & 0.0113 & 0.0028 & 0.0323 & 362 & $\mathrm{~cm}$ \\
\hline 916 & 3.602 & 0.000 & 18.67 & $3.602 \pm 0.034$ & 15.79 & 0.0165 & 0.0040 & 0.0338 & 362 & $\mathrm{~cm}$ \\
\hline 2456 & 0.211 & 0.212 & 79.10 & $0.422 \pm 0.003$ & 16.02 & 0.1025 & 0.0100 & 0.2835 & 362 & EW (V3) \\
\hline 2485 & 1.712 & 1.706 & 16.37 & $1.712 \pm 0.008$ & 15.71 & 0.0076 & 0.0033 & 0.0176 & 362 & $\mathrm{~cm}$ \\
\hline 2596 & 11.834 & 0.000 & 21.67 & $11.834 \pm 0.250$ & 16.18 & 0.0076 & 0.0039 & 0.0209 & 362 & $\mathrm{~cm}$ \\
\hline 2667 & 3.821 & 3.650 & 29.70 & $3.821 \pm 0.023$ & 15.82 & 0.0089 & 0.0041 & 0.0233 & 362 & $\mathrm{~cm}$ \\
\hline 2676 & 5.393 & 5.391 & 15.73 & $5.393 \pm 0.084$ & 15.50 & 0.0070 & 0.0030 & 0.0178 & 362 & $\mathrm{~cm}$ \\
\hline 2687 & 0.099 & 0.110 & 84.38 & $0.220 \pm 0.003$ & 16.18 & 0.1520 & 0.0100 & 0.4304 & 362 & $\delta$ Scuti (V6) \\
\hline 2701 & 3.981 & 0.000 & 17.20 & $3.981 \pm 0.040$ & 15.39 & 0.0050 & 0.0023 & 0.0143 & 362 & $\mathrm{~cm}$ \\
\hline 2742 & 5.135 & 5.155 & 20.30 & $5.155 \pm 0.065$ & 15.77 & 0.0092 & 0.0043 & 0.0270 & 362 & $\mathrm{~cm}$ \\
\hline 2835 & 5.473 & 5.391 & 15.46 & $5.391 \pm 0.077$ & 16.36 & 0.0160 & 0.0066 & 0.0399 & 362 & $\mathrm{~cm}$ \\
\hline 2874 & 10.209 & 10.000 & 16.20 & $10.209 \pm 0.317$ & 16.29 & 0.0076 & 0.0040 & 0.0222 & 362 & $\mathrm{~cm}$ \\
\hline 3031 & 7.065 & 0.000 & 21.78 & $7.065 \pm 0.081$ & 15.84 & 0.0099 & 0.0038 & 0.0235 & 362 & $\mathrm{~cm}$ \\
\hline 3040 & 2.687 & 2.674 & 23.95 & $2.687 \pm 0.017$ & 15.73 & 0.0093 & 0.0035 & 0.0259 & 362 & $\mathrm{~cm}$ \\
\hline 3089 & 5.990 & 0.000 & 22.62 & $5.990 \pm 0.058$ & 15.76 & 0.0065 & 0.0036 & 0.0196 & 362 & $\mathrm{~cm}$ \\
\hline 3146 & 3.742 & 0.000 & 21.34 & $3.742 \pm 0.031$ & 15.81 & 0.0095 & 0.0038 & 0.0275 & 362 & $\mathrm{~cm}$ \\
\hline 3246 & 0.042 & 0.044 & 19.63 & $0.044 \pm 0.003$ & 17.33 & 0.0404 & 0.0100 & 0.1128 & 362 & $\delta$ Scuti (KV10) \\
\hline \multirow{2}{*}{\multicolumn{11}{|c|}{ G-type periodic candidate members }} \\
\hline & & & & & & & & & & \\
\hline 427 & 5.672 & 5.500 & 19.01 & $5.500 \pm 0.200$ & 15.29 & 0.0068 & 0.0031 & 0.0196 & 362 & $\mathrm{~cm}$ \\
\hline 483 & 5.413 & 5.391 & 40.48 & $5.413 \pm 0.032$ & 15.55 & 0.0130 & 0.0028 & 0.0404 & 362 & $\mathrm{~cm}$ \\
\hline 2112 & 5.732 & 5.900 & 17.76 & $5.900 \pm 0.101$ & 16.66 & 0.0150 & 0.0054 & 0.0295 & 362 & $\mathrm{~cm}$ \\
\hline 2257 & 5.294 & 5.155 & 18.30 & $5.294 \pm 0.074$ & 16.03 & 0.0121 & 0.0050 & 0.0331 & 362 & $\mathrm{~cm}$ \\
\hline 2312 & 9.234 & 9.217 & 17.30 & $9.217 \pm 0.258$ & 16.72 & 0.0150 & 0.0068 & 0.0444 & 362 & $\mathrm{~cm}$ \\
\hline 2318 & 5.373 & 5.391 & 17.47 & $5.373 \pm 0.079$ & 15.50 & 0.0137 & 0.0040 & 0.0390 & 362 & $\mathrm{~cm}$ \\
\hline 2330 & 5.851 & 5.900 & 21.99 & $5.900 \pm 0.076$ & 17.00 & 0.0134 & 0.0068 & 0.0364 & 362 & $\mathrm{~cm}$ \\
\hline 2409 & 7.125 & 7.117 & 19.55 & $7.125 \pm 0.152$ & 17.10 & 0.0149 & 0.0068 & 0.0401 & 362 & $\mathrm{~cm}$ \\
\hline 2443 & 6.806 & 6.711 & 24.20 & $6.806 \pm 0.096$ & 16.58 & 0.0105 & 0.0048 & 0.0292 & 362 & $\mathrm{~cm}$ \\
\hline 2444 & 6.229 & 6.211 & 20.16 & $6.229 \pm 0.097$ & 16.18 & 0.0085 & 0.0038 & 0.0241 & 362 & $\mathrm{~cm}$ \\
\hline 2506 & 8.557 & 8.264 & 18.61 & $8.557 \pm 0.399$ & 17.58 & 0.0168 & 0.0093 & 0.0444 & 362 & $\mathrm{~cm}$ \\
\hline 2549 & 1.792 & 1.786 & 17.28 & $1.786 \pm 0.009$ & 16.24 & 0.0072 & 0.0037 & 0.0189 & 362 & $\mathrm{~cm}$ \\
\hline 2562 & 1.752 & 1.758 & 17.20 & $1.758 \pm 0.008$ & 16.78 & 0.0105 & 0.0051 & 0.0300 & 362 & $\mathrm{~cm}$ \\
\hline 2610 & 8.000 & 7.752 & 29.43 & $7.752 \pm 0.133$ & 16.83 & 0.0144 & 0.0063 & 0.0386 & 362 & $\mathrm{~cm}$ \\
\hline 2635 & 5.553 & 0.000 & 29.85 & $5.553 \pm 0.053$ & 16.31 & 0.0115 & 0.0047 & 0.0299 & 362 & $\mathrm{~cm}$ \\
\hline 2665 & 7.423 & 7.576 & 18.25 & $7.423 \pm 0.134$ & 17.05 & 0.0118 & 0.0062 & 0.0306 & 362 & $\mathrm{~cm}$ \\
\hline 2681 & 5.553 & 5.618 & 23.51 & $5.618 \pm 0.066$ & 16.27 & 0.0111 & 0.0042 & 0.0276 & 362 & $\mathrm{~cm}$ \\
\hline 2703 & 8.737 & 0.000 & 21.42 & $8.737 \pm 0.307$ & 16.10 & 0.0072 & 0.0034 & 0.0193 & 362 & $\mathrm{~cm}$ \\
\hline 2728 & 6.567 & 0.000 & 22.47 & $6.567 \pm 0.095$ & 16.43 & 0.0124 & 0.0062 & 0.0367 & 362 & $\mathrm{~cm}$ \\
\hline 2743 & 1.133 & 0.000 & 36.69 & $1.155 \pm 0.003$ & 16.92 & 0.0264 & 0.0075 & 0.0740 & 362 & $\mathrm{~cm}$ \\
\hline 2763 & 1.314 & 1.316 & 17.72 & $1.314 \pm 0.004$ & 16.89 & 0.0164 & 0.0067 & 0.0457 & 362 & $\mathrm{~cm}$ \\
\hline 2832 & 0.279 & 0.279 & 51.70 & $0.558 \pm 0.003$ & 15.95 & 0.0899 & 0.0100 & 0.2720 & 362 & EW (V4) \\
\hline 2843 & 4.876 & 4.938 & 17.61 & $4.876 \pm 0.067$ & 17.04 & 0.0132 & 0.0061 & 0.0373 & 362 & $\mathrm{~cm}$ \\
\hline 2847 & 1.274 & 0.000 & 18.16 & $1.274 \pm 0.004$ & 15.62 & 0.0065 & 0.0029 & 0.0181 & 362 & $\mathrm{~cm}$ \\
\hline 2859 & 6.329 & 6.211 & 26.34 & $6.329 \pm 0.072$ & 16.30 & 0.0081 & 0.0037 & 0.0230 & 362 & $\mathrm{~cm}$ \\
\hline 2895 & 1.786 & 0.000 & 20.97 & $1.786 \pm 0.004$ & 16.05 & 0.0076 & 0.0036 & 0.0195 & 362 & $\mathrm{~cm}$ \\
\hline 2947 & 3.702 & 3.984 & 20.64 & $3.984 \pm 0.030$ & 15.83 & 0.0052 & 0.0033 & 0.0153 & 362 & $\mathrm{~cm}$ \\
\hline 2948 & 5.274 & 5.155 & 21.02 & $5.274 \pm 0.067$ & 16.12 & 0.0077 & 0.0039 & 0.0217 & 362 & $\mathrm{~cm}$ \\
\hline
\end{tabular}


Table 2. continued.

\begin{tabular}{|c|c|c|c|c|c|c|c|c|c|c|}
\hline ID & $\begin{array}{r}P_{\text {Scargle }} \\
\text { (d) }\end{array}$ & $\begin{array}{r}P_{\text {CLEAN }} \\
\text { (d) }\end{array}$ & $\overline{P_{\mathrm{N}}}$ & $\begin{array}{r}P \pm \Delta P \\
(\mathrm{~d})\end{array}$ & $\begin{array}{l}V_{\text {mean }} \\
\text { (mag) }\end{array}$ & $\begin{array}{l}\sigma_{\mathrm{TOT}} \\
(\mathrm{mag})\end{array}$ & $\begin{array}{l}\text { Accuracy } \\
\text { (mag) }\end{array}$ & $\begin{array}{l}\Delta V \\
(\mathrm{mag})\end{array}$ & \# obs. & Note \\
\hline 2968 & 6.349 & 6.211 & 22.83 & $6.211 \pm 0.102$ & 17.02 & 0.0149 & 0.0067 & 0.0434 & 362 & $\mathrm{~cm}$ \\
\hline 2974 & 1.811 & 1.813 & 27.00 & $1.813 \pm 0.007$ & 16.33 & 0.0086 & 0.0046 & 0.0251 & 362 & $\mathrm{~cm}$ \\
\hline 2995 & 0.493 & 0.000 & 22.30 & $0.986 \pm 0.003$ & 16.41 & 0.0315 & 0.0100 & 0.0882 & 362 & EA (KV12) \\
\hline 2996 & 6.110 & 6.211 & 18.56 & $6.211 \pm 0.087$ & 17.11 & 0.0112 & 0.0074 & 0.0277 & 362 & $\mathrm{~cm}$ \\
\hline 2997 & 7.045 & 7.117 & 35.98 & $7.117 \pm 0.045$ & 16.78 & 0.0143 & 0.0052 & 0.0407 & 362 & $\mathrm{~cm}$ \\
\hline 3003 & 6.906 & 0.000 & 27.28 & $6.906 \pm 0.063$ & 16.99 & 0.0137 & 0.0059 & 0.0394 & 362 & $\mathrm{~cm}$ \\
\hline 3008 & 2.110 & 2.105 & 30.42 & $2.105 \pm 0.008$ & 16.97 & 0.0188 & 0.0054 & 0.0524 & 362 & $\mathrm{~cm}$ \\
\hline 3021 & 10.110 & 10.000 & 18.42 & $10.000 \pm 0.288$ & 15.70 & 0.0403 & 0.0089 & 0.0975 & 362 & $\mathrm{~cm}$ \\
\hline 3030 & 1.951 & 1.963 & 23.38 & $1.963 \pm 0.008$ & 17.24 & 0.0116 & 0.0064 & 0.0303 & 362 & $\mathrm{~cm}$ \\
\hline 3094 & 3.224 & 0.000 & 46.64 & $3.224 \pm 0.009$ & 16.72 & 0.0442 & 0.0087 & 0.1196 & 362 & $\mathrm{~cm}$ \\
\hline 3110 & 2.090 & 2.105 & 25.89 & $2.105 \pm 0.009$ & 16.22 & 0.0110 & 0.0042 & 0.0297 & 362 & $\mathrm{~cm}$ \\
\hline 3157 & 5.771 & 5.900 & 33.64 & $5.900 \pm 0.042$ & 16.21 & 0.0110 & 0.0050 & 0.0329 & 362 & $\mathrm{~cm}$ \\
\hline 3208 & 0.876 & 0.000 & 15.62 & $0.876 \pm 0.002$ & 16.97 & 0.0124 & 0.0075 & 0.0353 & 362 & $\mathrm{~cm}$ \\
\hline 3209 & 5.771 & 5.900 & 21.75 & $5.900 \pm 0.086$ & 16.34 & 0.0155 & 0.0058 & 0.0434 & 362 & $\mathrm{~cm}$ \\
\hline 3230 & 8.339 & 8.032 & 16.24 & $8.339 \pm 0.332$ & 17.12 & 0.0148 & 0.0086 & 0.0408 & 362 & $\mathrm{~cm}$ \\
\hline 3247 & 12.139 & 11.834 & 18.62 & $11.834 \pm 0.383$ & 16.82 & 0.0136 & 0.0071 & 0.0392 & 362 & $\mathrm{~cm}$ \\
\hline 3294 & 7.344 & 7.117 & 22.84 & $7.344 \pm 0.128$ & 16.62 & 0.0245 & 0.0109 & 0.0618 & 362 & $\mathrm{~cm}$ \\
\hline 3512 & 6.508 & 0.000 & 17.07 & $6.508 \pm 0.112$ & 17.18 & 0.0135 & 0.0088 & 0.0331 & 362 & $\mathrm{~cm}$ \\
\hline 3617 & 6.309 & 0.000 & 23.54 & $6.309 \pm 0.080$ & 16.62 & 0.0083 & 0.0051 & 0.0236 & 362 & $\mathrm{~cm}$ \\
\hline 3713 & 3.642 & 0.000 & 20.24 & $3.642 \pm 0.100$ & 16.92 & 0.0158 & 0.0072 & 0.0437 & 362 & $\mathrm{~cm}$ \\
\hline 3714 & 5.900 & 0.000 & 22.15 & $5.900 \pm 0.030$ & 16.98 & 0.0140 & 0.0071 & 0.0384 & 362 & $\mathrm{~cm}$ \\
\hline 3724 & 0.279 & 0.279 & 0.00 & $0.558 \pm 0.003$ & 16.09 & 0.1361 & 0.0100 & 0.3652 & 361 & RRc (V5) \\
\hline 3725 & 6.110 & 0.000 & 15.51 & $6.110 \pm 0.088$ & 17.03 & 0.0159 & 0.0083 & 0.0421 & 362 & $\mathrm{~cm}$ \\
\hline 3866 & 5.175 & 5.155 & 23.09 & $5.155 \pm 0.064$ & 15.96 & 0.0138 & 0.0047 & 0.0404 & 362 & $\mathrm{~cm}$ \\
\hline 4035 & 4.040 & 0.000 & 16.30 & $4.040 \pm 0.048$ & 15.80 & 0.0094 & 0.0040 & 0.0251 & 361 & $\mathrm{~cm}$ \\
\hline 4181 & 0.179 & 0.179 & 87.35 & $0.358 \pm 0.003$ & 17.63 & 0.1571 & 0.0100 & 0.4294 & 362 & EW (V7) \\
\hline 4654 & 0.177 & 0.178 & 16.00 & $0.354 \pm 0.003$ & 18.55 & 0.0850 & 0.0100 & 0.2372 & 362 & EW (KV15) \\
\hline 4708 & 0.167 & 0.168 & 35.90 & $0.335 \pm 0.003$ & 18.05 & 0.0671 & 0.0100 & 0.2024 & 361 & EW (KV14) \\
\hline 5082 & 5.990 & 5.900 & 31.76 & $5.990 \pm 0.056$ & 16.50 & 0.0171 & 0.0052 & 0.0424 & 362 & $\mathrm{~cm}$ \\
\hline 5906 & 0.148 & 0.147 & 23.60 & $0.295 \pm 0.003$ & 20.45 & 0.2087 & 0.0100 & 0.5837 & 357 & EW (KV16) \\
\hline 6462 & 3.722 & 0.000 & 20.73 & $3.722 \pm 0.032$ & 17.90 & 0.0457 & 0.0129 & 0.1280 & 362 & $\mathrm{~cm}$ \\
\hline 7941 & 0.169 & 0.145 & 14.73 & $0.290 \pm 0.003$ & 17.65 & 0.0538 & 0.0100 & 0.1458 & 362 & EW (KV13) \\
\hline \multicolumn{11}{|c|}{ K-type periodic candidate members } \\
\hline 2321 & 11.881 & 11.834 & 19.13 & $11.881 \pm 0.325$ & 17.20 & 0.0152 & 0.0080 & 0.0425 & 362 & $\mathrm{~cm}$ \\
\hline 2395 & 3.992 & 0.000 & 21.68 & $3.992 \pm 0.020$ & 17.19 & 0.0186 & 0.0069 & 0.0540 & 362 & $\mathrm{~cm}$ \\
\hline 2408 & 7.224 & 0.000 & 27.42 & $7.224 \pm 0.071$ & 17.34 & 0.0157 & 0.0085 & 0.0430 & 362 & $\mathrm{~cm}$ \\
\hline 2472 & 1.473 & 0.000 & 34.77 & $1.473 \pm 0.004$ & 17.59 & 0.0233 & 0.0081 & 0.0666 & 362 & $\mathrm{~cm}$ \\
\hline 2473 & 6.766 & 0.000 & 24.30 & $6.766 \pm 0.076$ & 17.13 & 0.0116 & 0.0072 & 0.0329 & 362 & $\mathrm{~cm}$ \\
\hline 2509 & 14.468 & 13.158 & 15.82 & $14.468 \pm 0.720$ & 17.07 & 0.0127 & 0.0071 & 0.0354 & 362 & $\mathrm{~cm}$ \\
\hline 2541 & 7.145 & 0.000 & 24.40 & $7.145 \pm 0.116$ & 17.53 & 0.0188 & 0.0087 & 0.0518 & 362 & $\mathrm{~cm}$ \\
\hline 2595 & 8.737 & 0.000 & 21.84 & $8.737 \pm 0.333$ & 17.27 & 0.0159 & 0.0068 & 0.0438 & 362 & $\mathrm{~cm}$ \\
\hline 2597 & 6.289 & 6.211 & 16.83 & $6.211 \pm 0.094$ & 17.46 & 0.0157 & 0.0071 & 0.0434 & 362 & $\mathrm{~cm}$ \\
\hline 2618 & 7.117 & 0.000 & 18.67 & $7.117 \pm 0.200$ & 16.93 & 0.0116 & 0.0062 & 0.0330 & 362 & $\mathrm{~cm}$ \\
\hline 2639 & 7.543 & 7.576 & 19.30 & $7.576 \pm 0.147$ & 17.51 & 0.0126 & 0.0066 & 0.0352 & 362 & $\mathrm{~cm}$ \\
\hline 2646 & 7.204 & 0.000 & 16.12 & $7.204 \pm 0.161$ & 17.81 & 0.0180 & 0.0100 & 0.0518 & 362 & $\mathrm{~cm}$ \\
\hline 2734 & 7.443 & 7.576 & 15.91 & $7.576 \pm 0.152$ & 17.08 & 0.0136 & 0.0067 & 0.0358 & 362 & $\mathrm{~cm}$ \\
\hline 2890 & 8.239 & 8.065 & 24.48 & $8.239 \pm 0.233$ & 17.63 & 0.0195 & 0.0087 & 0.0510 & 362 & $\mathrm{~cm}$ \\
\hline 2971 & 19.980 & 0.000 & 17.85 & $19.980 \pm 0.964$ & 16.34 & 0.0088 & 0.0039 & 0.0233 & 362 & $\mathrm{~cm}$ \\
\hline 3082 & 6.806 & 6.711 & 28.90 & $6.806 \pm 0.061$ & 17.32 & 0.0142 & 0.0075 & 0.0432 & 362 & $\mathrm{~cm}$ \\
\hline 3236 & 8.657 & 8.097 & 21.70 & $8.657 \pm 0.463$ & 16.94 & 0.0148 & 0.0068 & 0.0422 & 362 & $\mathrm{~cm}$ \\
\hline 3245 & 5.391 & 0.000 & 27.45 & $5.391 \pm 0.020$ & 17.28 & 0.0215 & 0.0086 & 0.0577 & 362 & $\mathrm{~cm}$ \\
\hline 3582 & 4.876 & 4.938 & 24.20 & $4.876 \pm 0.058$ & 17.28 & 0.0179 & 0.0076 & 0.0509 & 362 & $\mathrm{~cm}$ \\
\hline 3638 & 6.189 & 0.000 & 16.88 & $6.189 \pm 0.092$ & 16.76 & 0.0151 & 0.0075 & 0.0415 & 362 & $\mathrm{~cm}$ \\
\hline 3702 & 7.125 & 7.117 & 16.69 & $7.125 \pm 0.141$ & 16.97 & 0.0145 & 0.0056 & 0.0299 & 362 & $\mathrm{~cm}$ \\
\hline 3712 & 7.145 & 7.117 & 20.74 & $7.145 \pm 0.125$ & 16.85 & 0.0156 & 0.0077 & 0.0450 & 362 & $\mathrm{~cm}$ \\
\hline 4103 & 5.393 & 0.855 & 39.13 & $5.393 \pm 0.021$ & 16.96 & 0.0275 & 0.0095 & 0.0777 & 362 & $\mathrm{~cm}$ \\
\hline 4134 & 5.680 & 5.680 & 14.66 & $5.680 \pm 0.063$ & 17.37 & 0.0131 & 0.0079 & 0.0343 & 362 & $\mathrm{~cm}$ \\
\hline 4423 & 7.901 & 8.032 & 22.91 & $7.901 \pm 0.155$ & 17.96 & 0.0267 & 0.0136 & 0.0718 & 362 & $\mathrm{~cm}$ \\
\hline 4481 & 9.055 & 0.000 & 30.99 & $9.055 \pm 0.165$ & 17.96 & 0.0252 & 0.0118 & 0.0704 & 362 & $\mathrm{~cm}$ \\
\hline 4822 & 2.428 & 2.445 & 19.94 & $2.428 \pm 0.013$ & 18.89 & 0.0488 & 0.0248 & 0.1343 & 362 & $\mathrm{~cm}$ \\
\hline 6040 & 1.035 & 0.000 & 36.90 & $1.035 \pm 0.003$ & 18.98 & 0.0813 & 0.0295 & 0.2338 & 362 & $\mathrm{~cm}$ \\
\hline
\end{tabular}


S. Messina et al.: Rotation and variability in NGC 2099, Online Material p 5
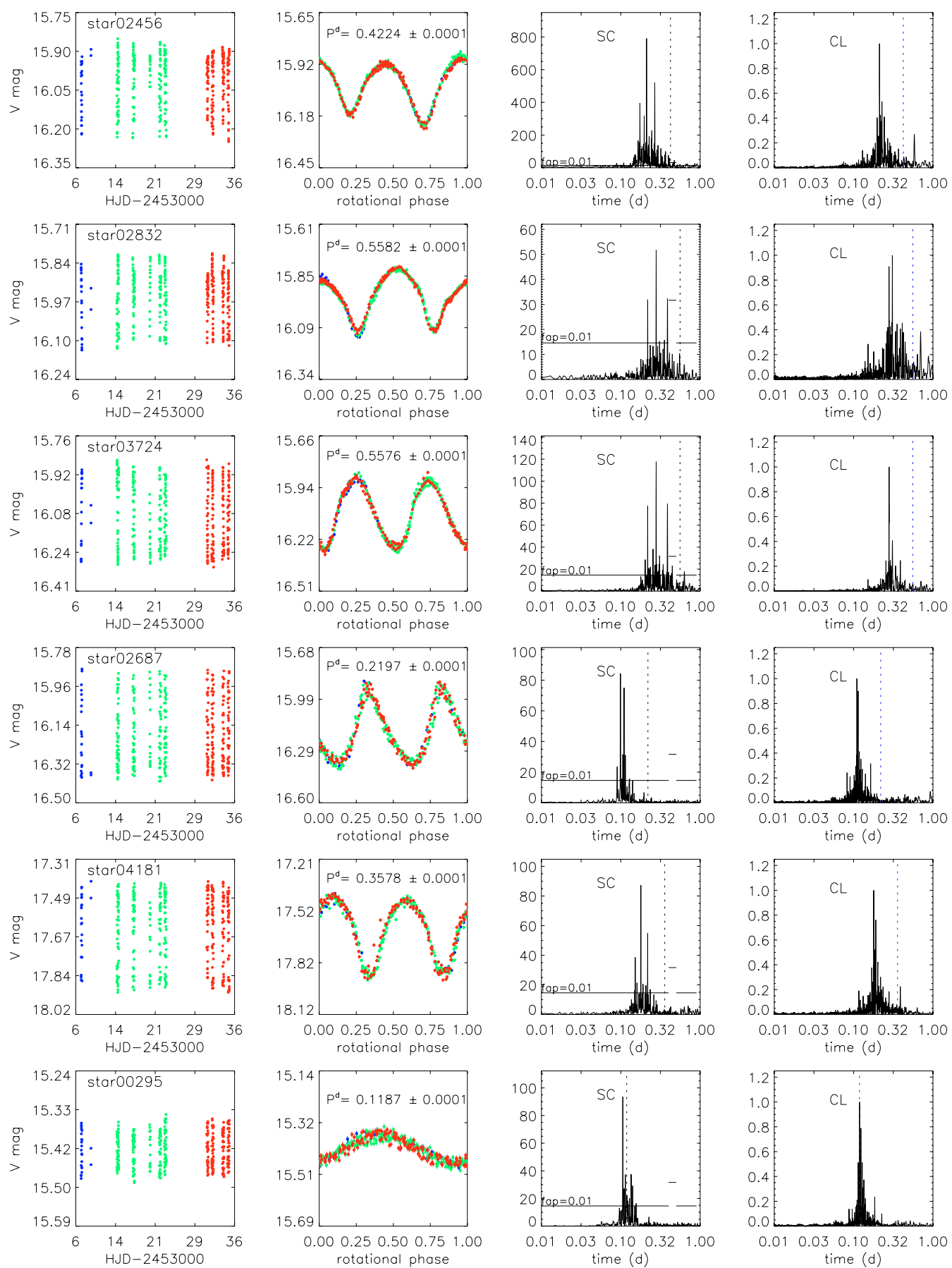

Fig. 11. Results of Fourier analysis of periodic variables in M 37 previously discovered by Kiss et al. (2001) and Kang et al. (2007) surveys. From left panel: $V$-band time series; phased light curve; Scargle periodogram and CLEAN periodogram. In case of eclipsing binaries the periodicity detected by either Scargle or CLEAN falls at exactly half the orbital period (vertical dashed line). See Sect. 3.3 for a detailed description. 
S. Messina et al.: Rotation and variability in NGC 2099, Online Material p 6
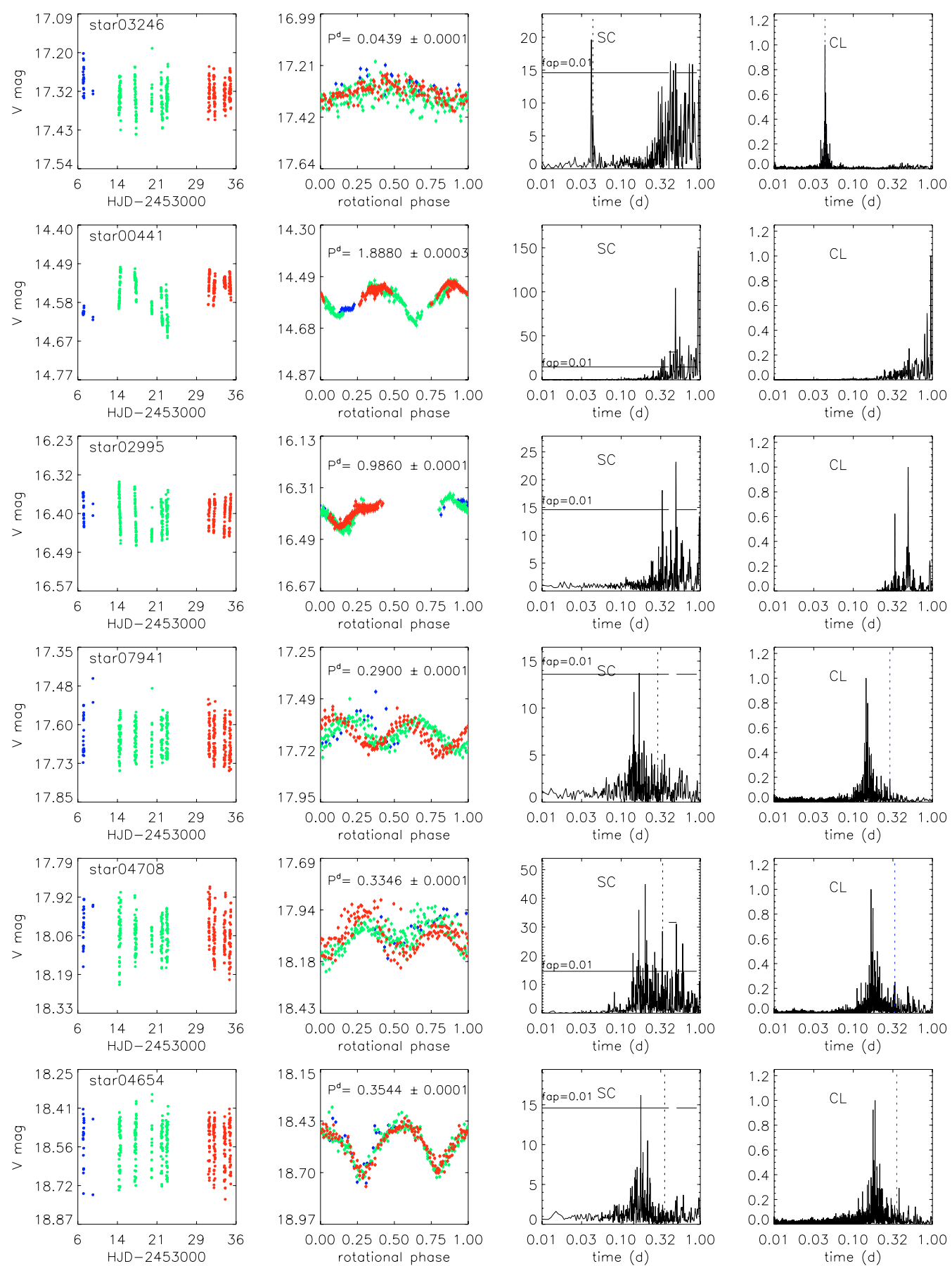

Fig. 12. As in Fig. 11. 
S. Messina et al.: Rotation and variability in NGC 2099, Online Material $p 7$
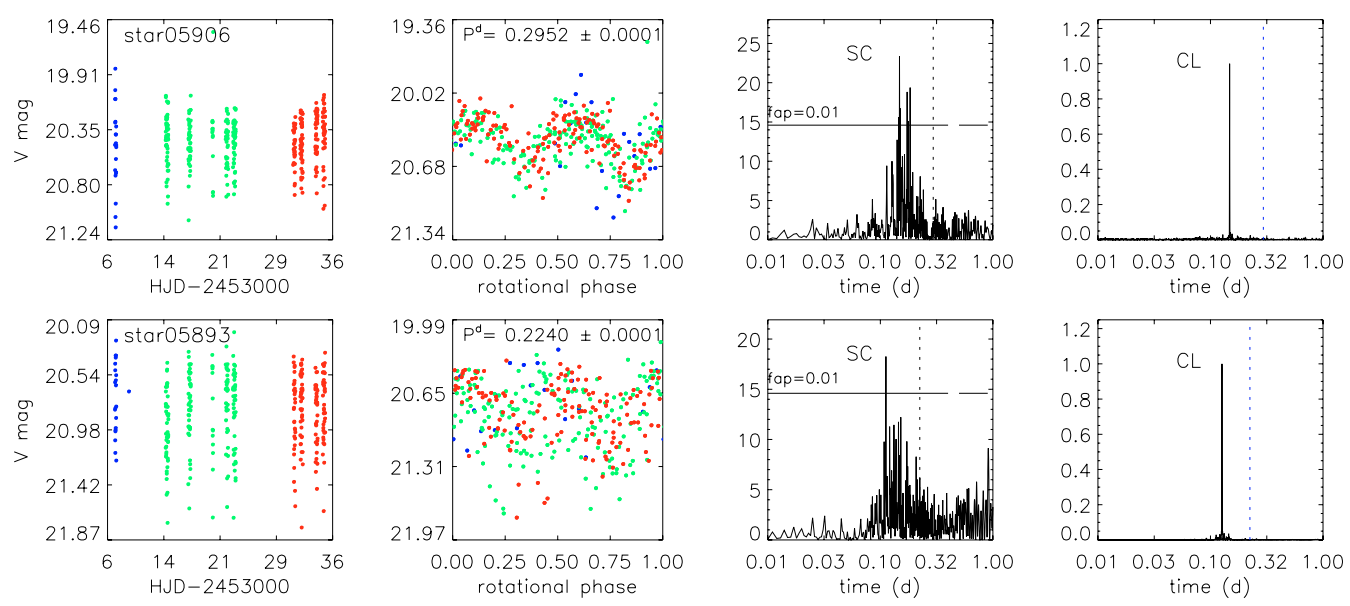

Fig. 13. As in Fig. 11. 
S. Messina et al.: Rotation and variability in NGC 2099, Online Material $p 8$
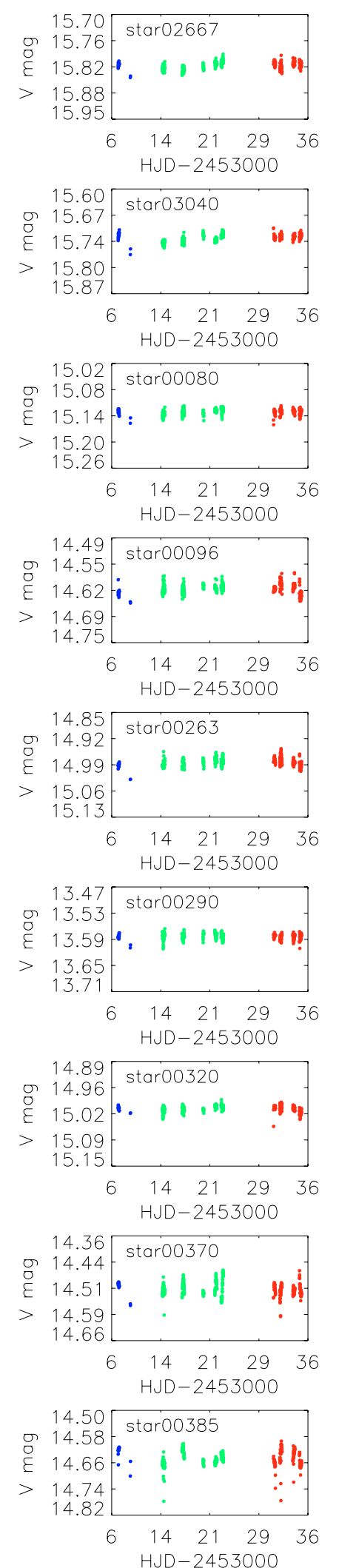
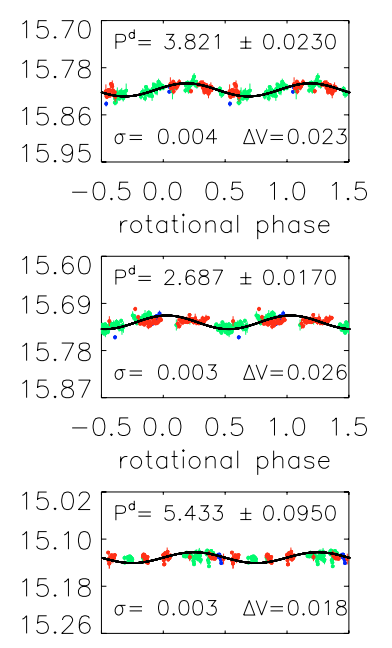

$\begin{array}{lllll}-0.5 & 0.0 & 0.5 & 1.0 & 1.5\end{array}$ rotational phase
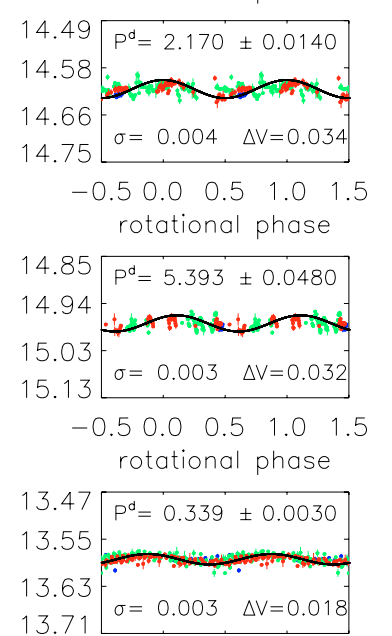

$\begin{array}{lllll}-0.5 & 0.0 & 0.5 & 1.0 & 1.5\end{array}$ rotational phase
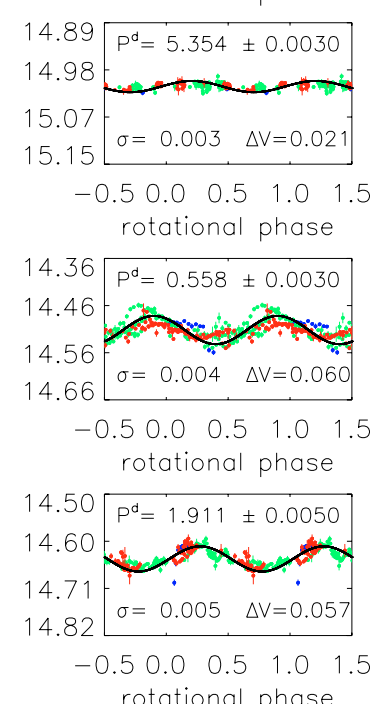
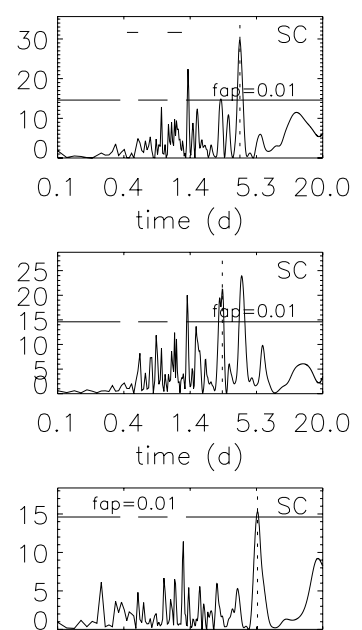

$\begin{array}{lllll}0.1 & 0.4 & 1.4 & 5.3 & 20.0\end{array}$ time (d)
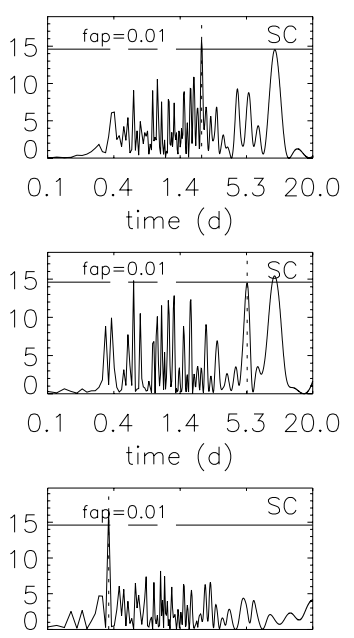

$\begin{array}{lllll}0.1 & 0.4 & 1.4 & 5.3 & 20.0\end{array}$ time (d)
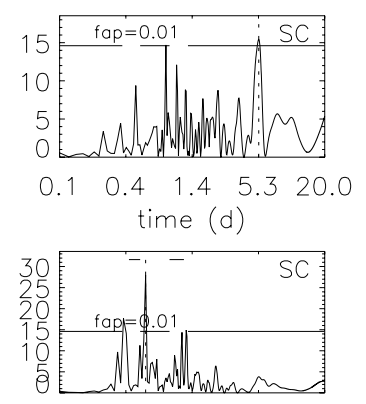

$\begin{array}{llllll}0.1 & 0.4 & 1.4 & 5.3 & 20.0\end{array}$ time (d)

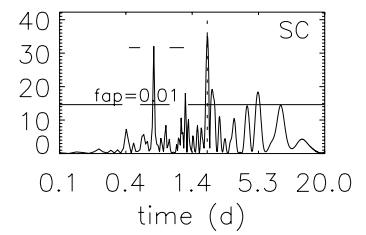

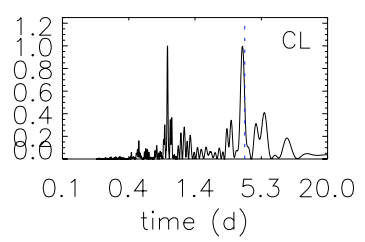
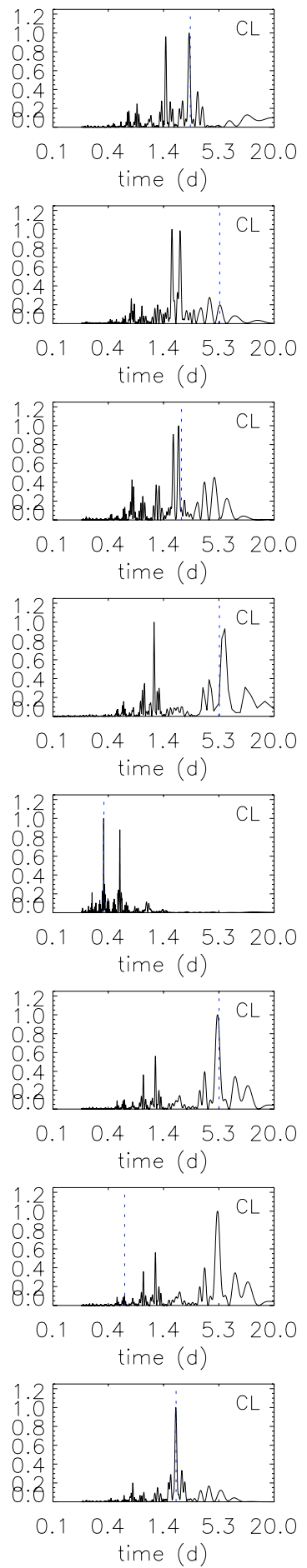

Fig. 14. F-type periodic candidate cluster members. From left panel: $V$-band time series; phased light curve; Scargle and CLEAN periodogram. See Sect. 3.3 for a detailed description. 
S. Messina et al.: Rotation and variability in NGC 2099, Online Material p 9

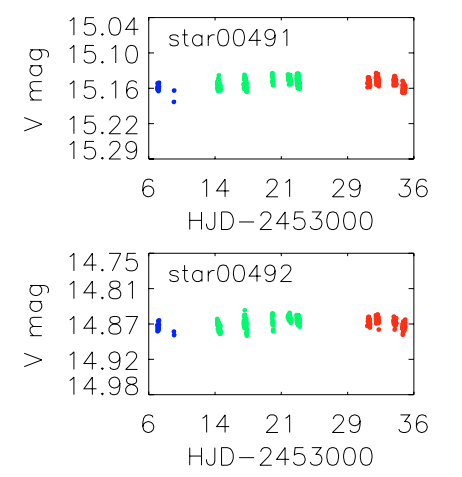

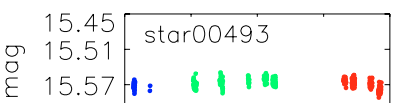

$>15.62$

$\begin{array}{lllll}6 & 14 & 21 & 29 & 36\end{array}$ HJD -2453000
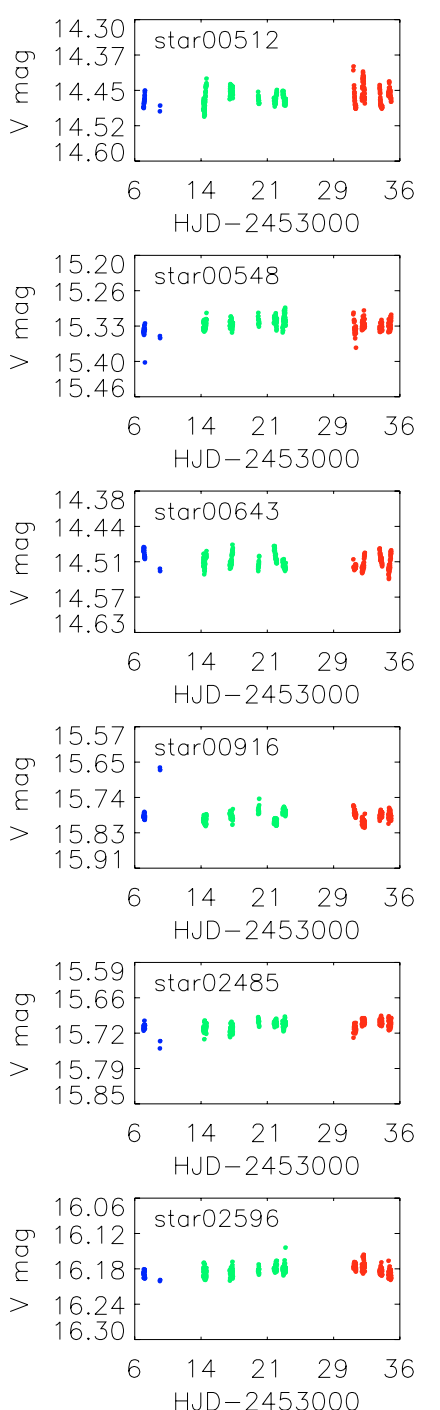
HJD -2453000
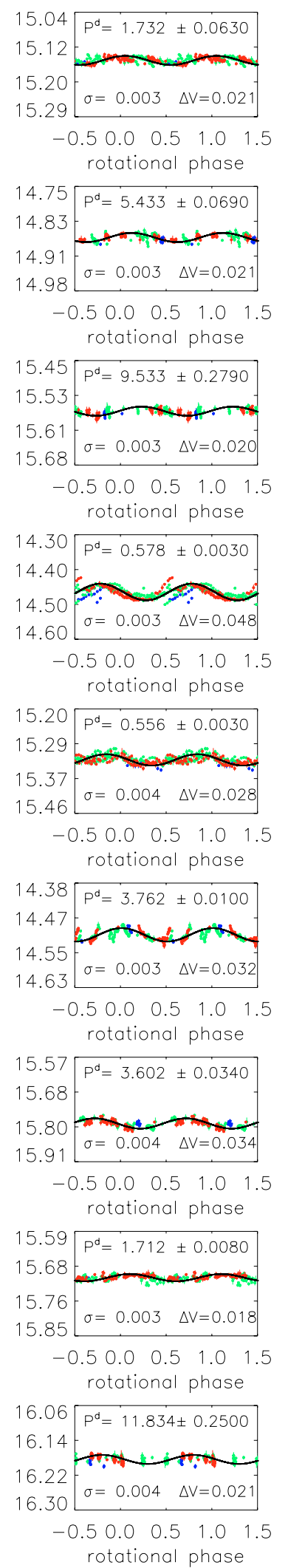
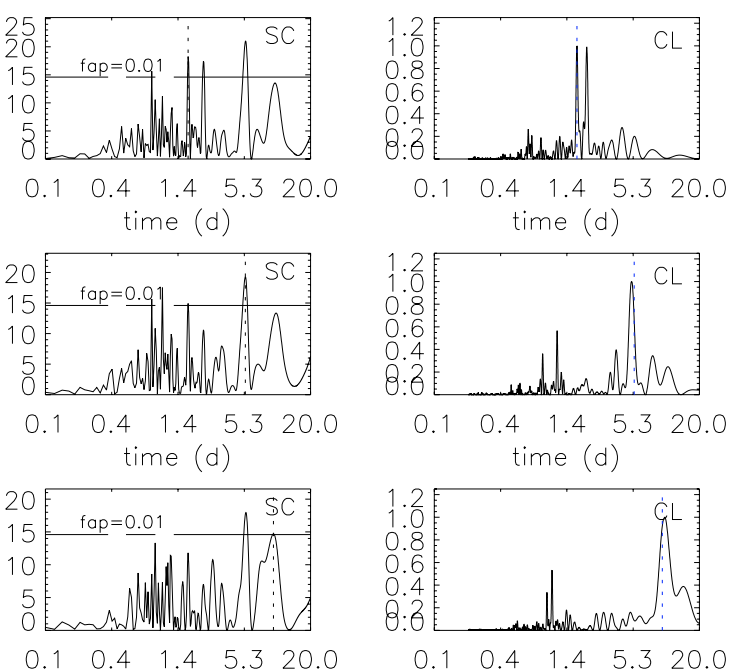
time (d)
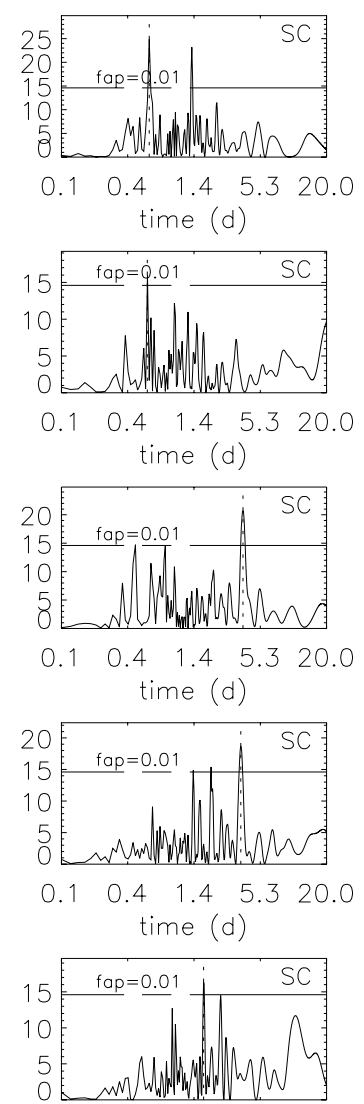

$\begin{array}{lllll}0.1 & 0.4 & 1.4 & 5.3 & 20.0\end{array}$ time (d)
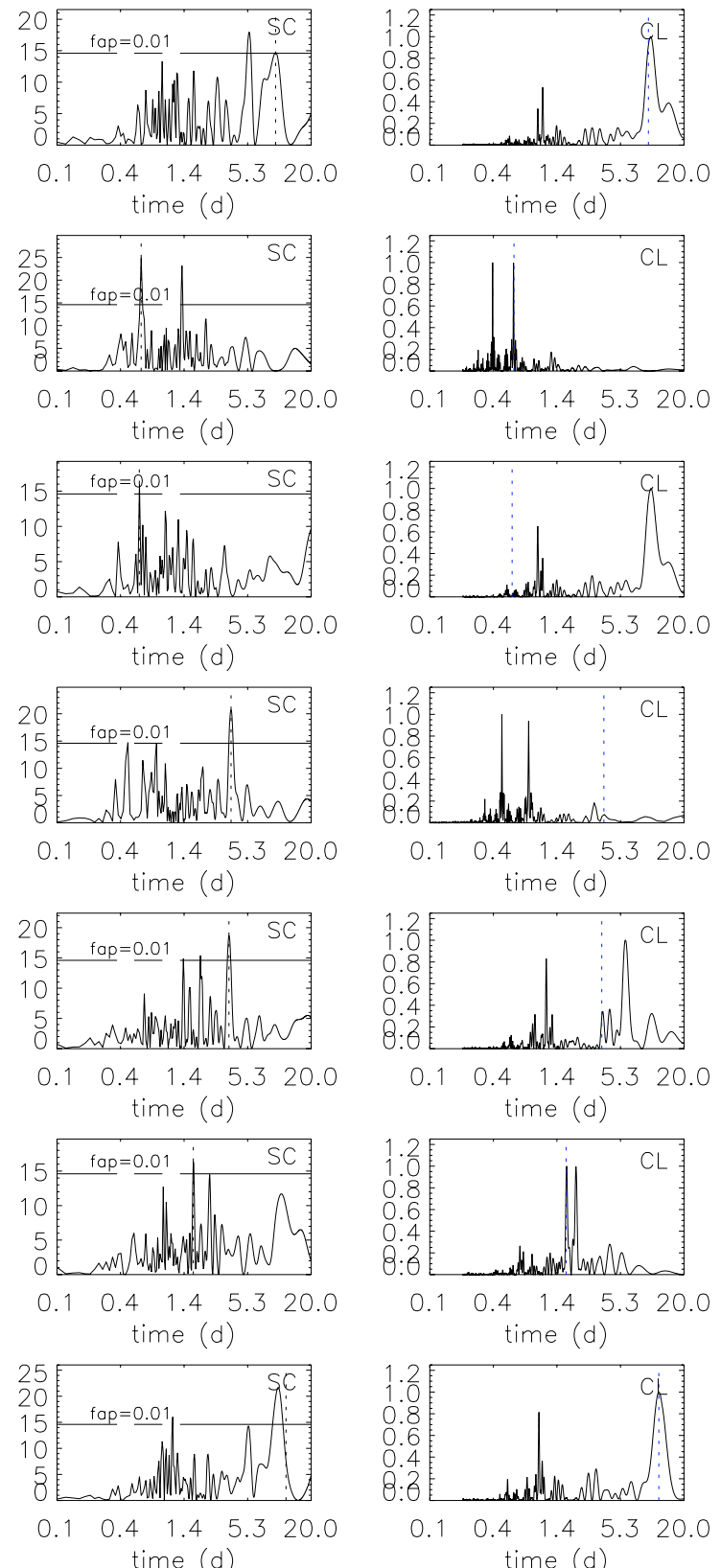
time (d)
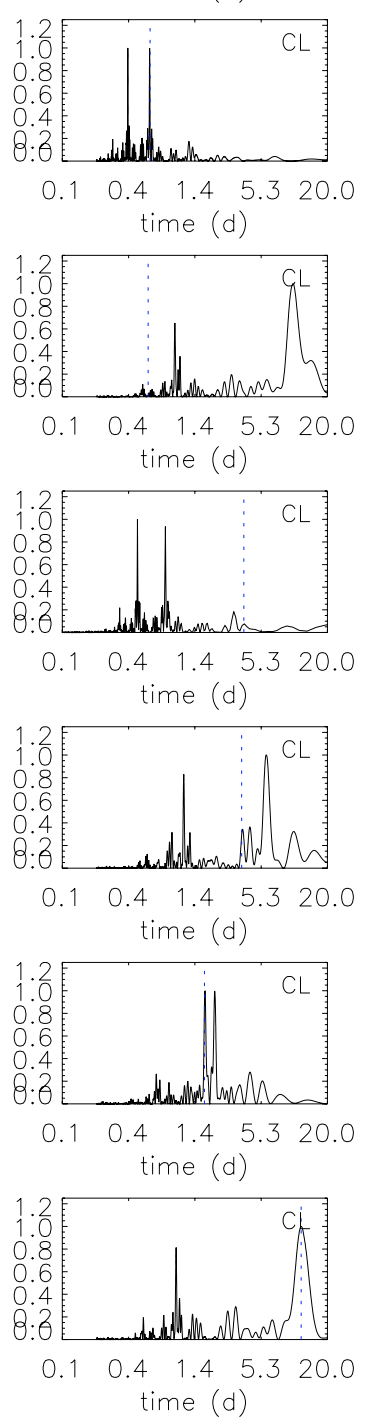

Fig. 15. As in Fig. 14. 
S. Messina et al.: Rotation and variability in NGC 2099, Online Material $p 10$
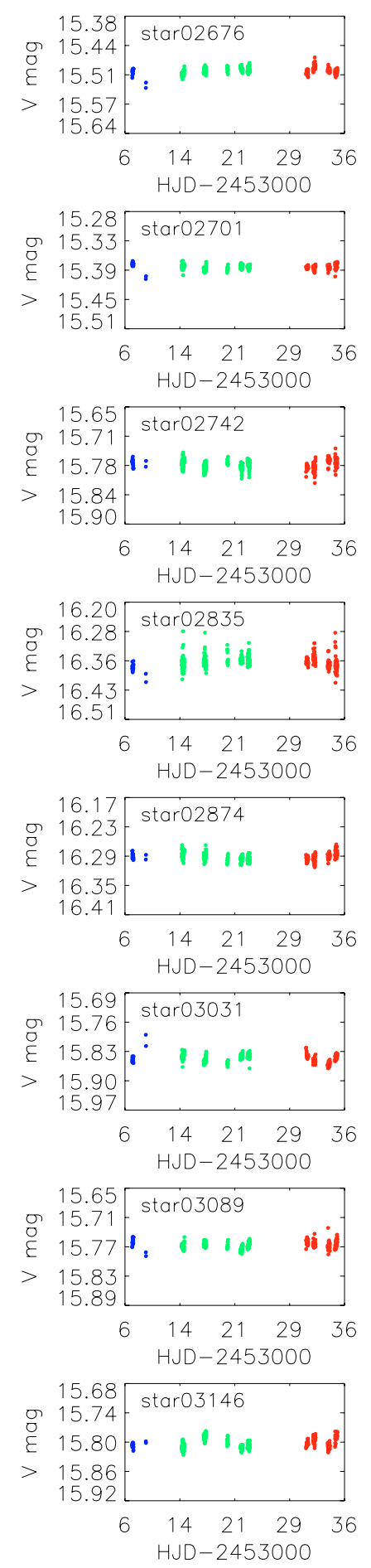

Fig. 16. As in Fig. 14.
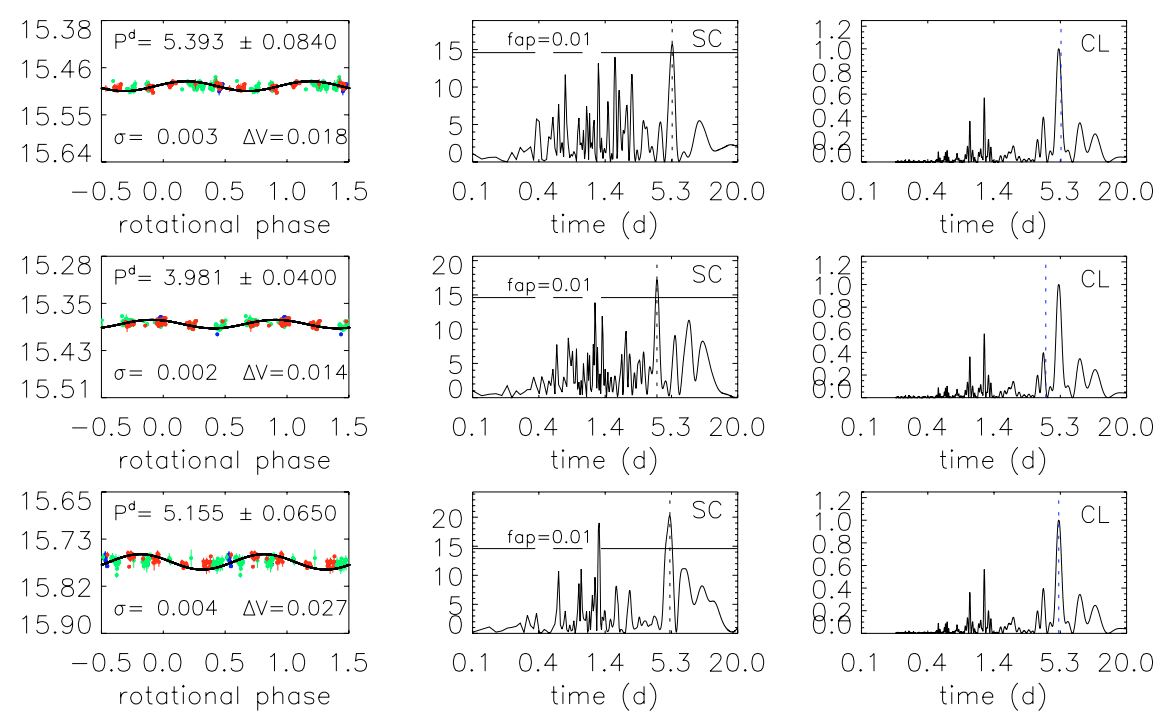

$\begin{array}{lllll}0.1 & 0.4 & 1.4 & 5.3 & 20.0\end{array}$ time (d)
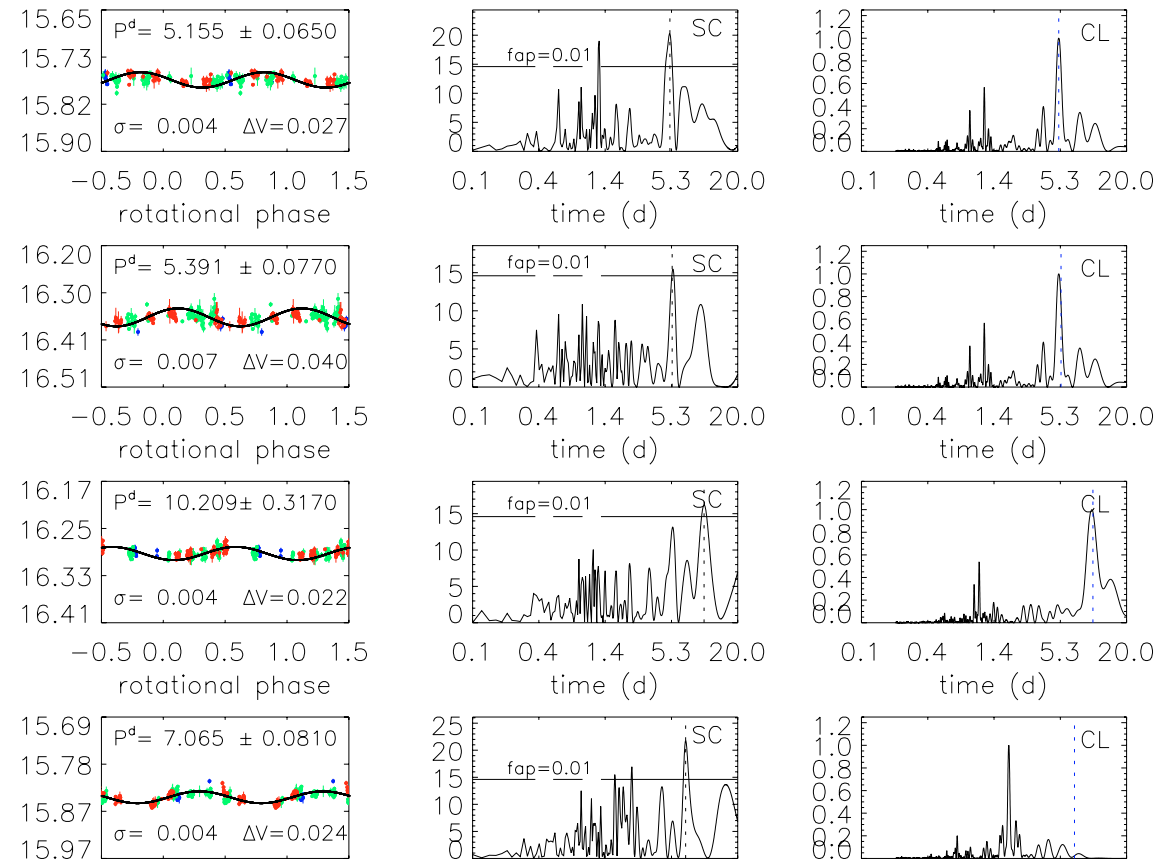

$\begin{array}{lllll}-0.5 & 0.0 & 0.5 & 1.0 & 1.5\end{array}$ rotational phase
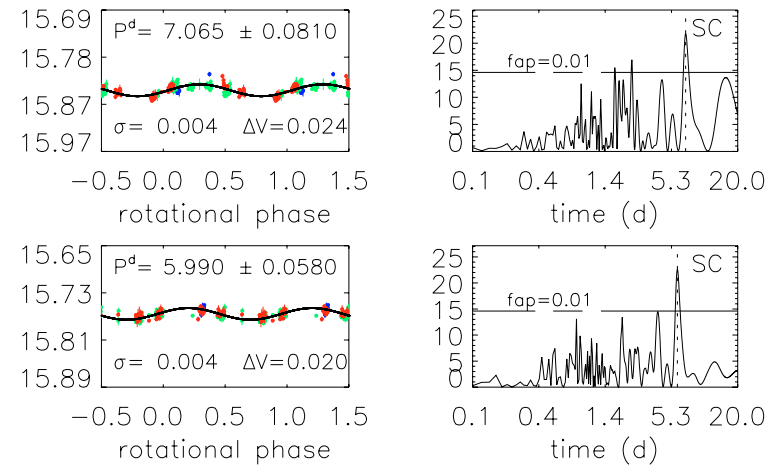

$\begin{array}{lllll}0.1 & 0.4 & 1.4 & 5.3 & 20.0\end{array}$ time (d)
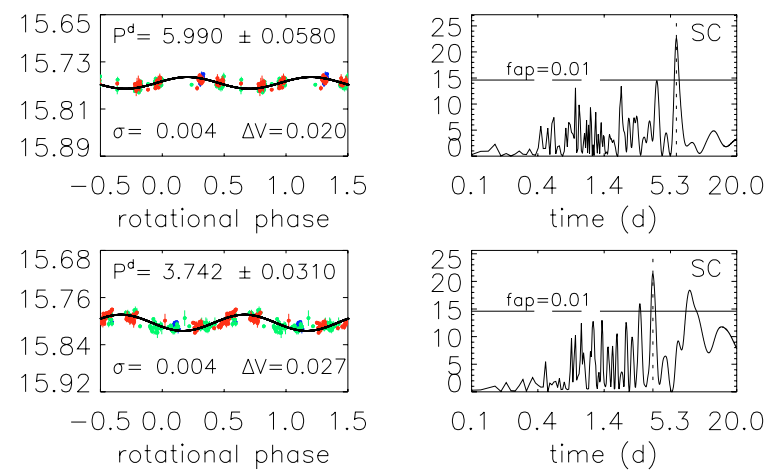

$\begin{array}{lllll}0.1 & 0.4 & 1.4 & 5.3 & 20.0\end{array}$ time (d)
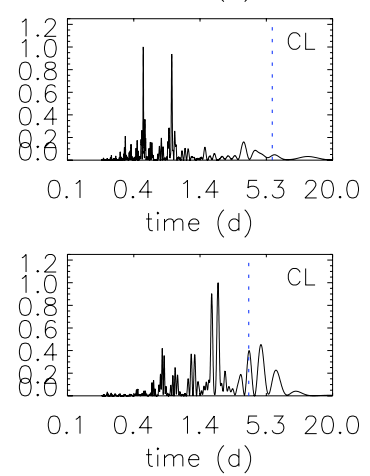
S. Messina et al.: Rotation and variability in NGC 2099, Online Material p 11
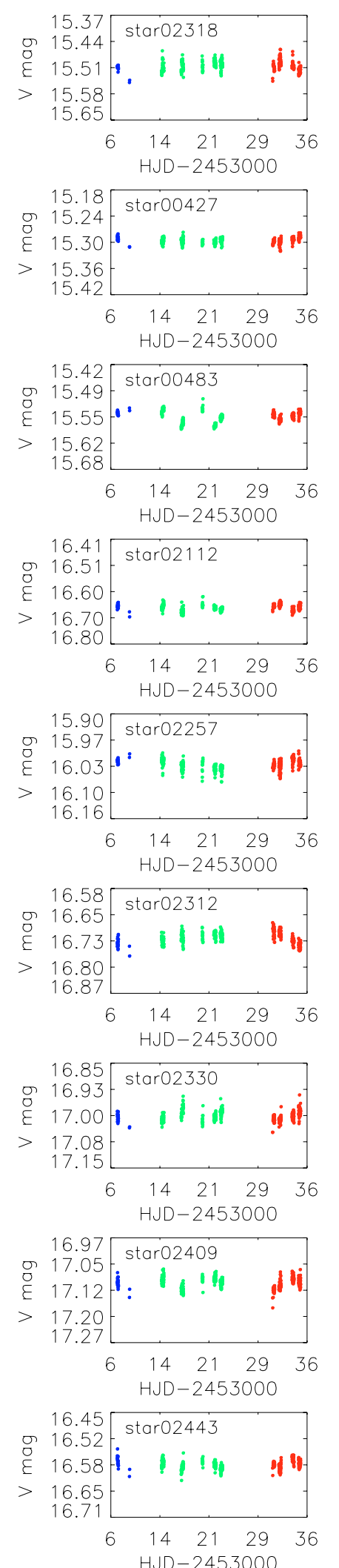
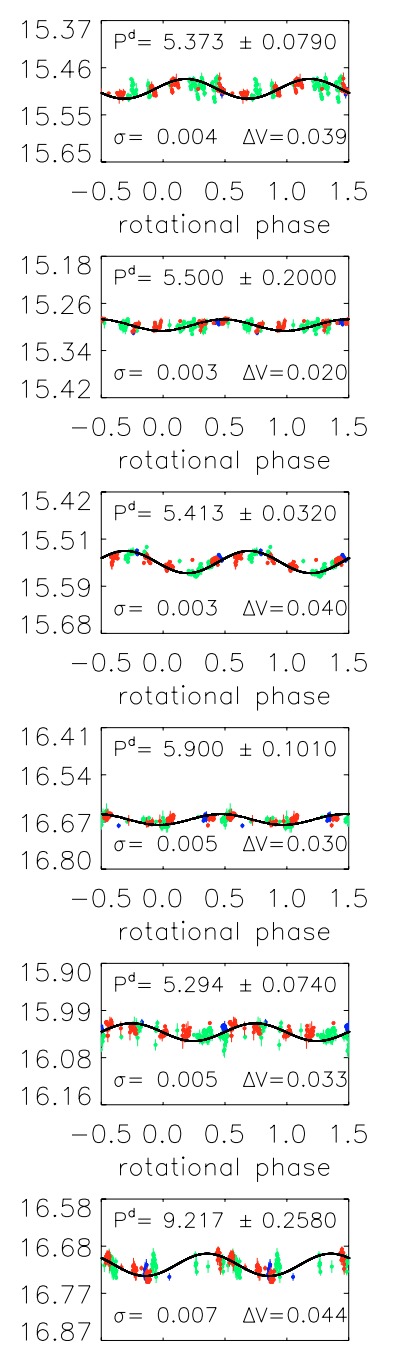

$-0.50 .0 \quad 0.5 \quad 1.0 \quad 1.5$ rotational phase
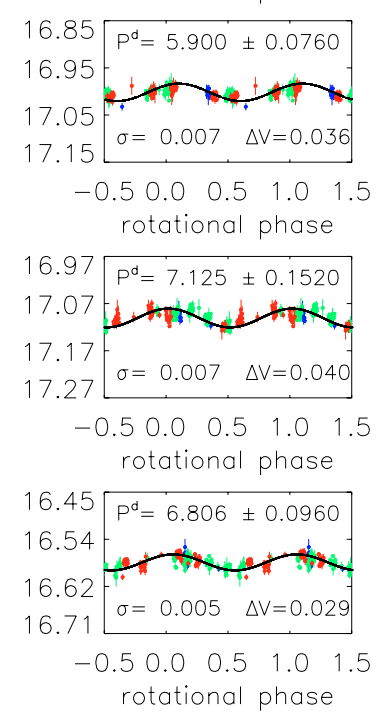
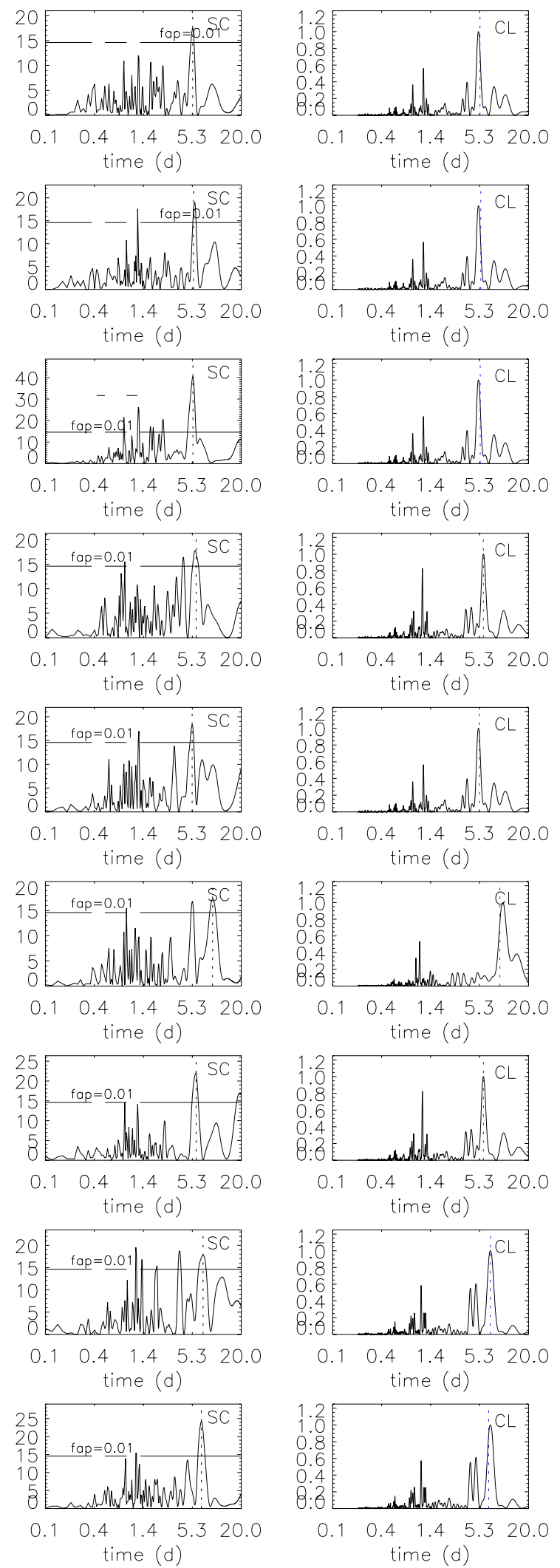

Fig. 17. G-type periodic candidate cluster members. From left panel: V-band time series; phased light curve; Scargle and CLEAN periodogram. See Sect. 3.3 for a detailed description. 
S. Messina et al.: Rotation and variability in NGC 2099, Online Material p 12
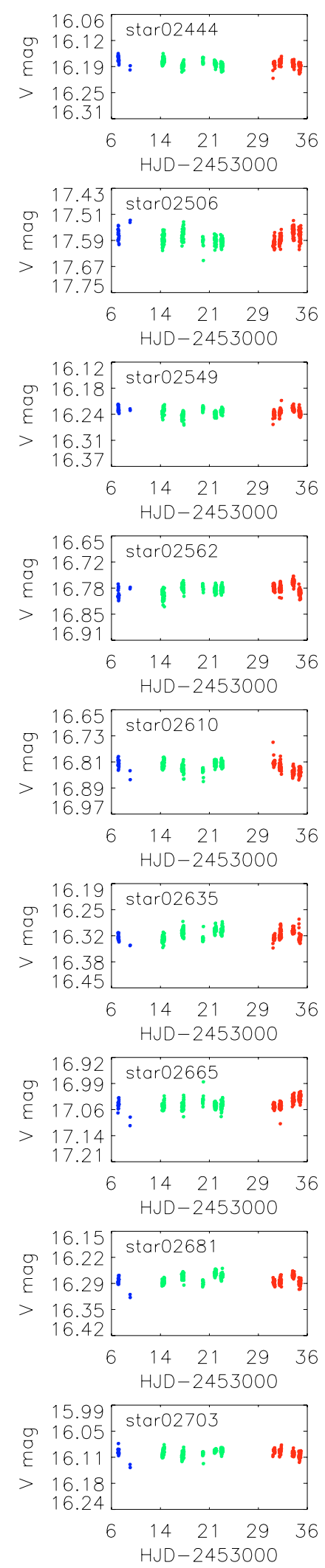

Fig. 18. As in Fig. 17.
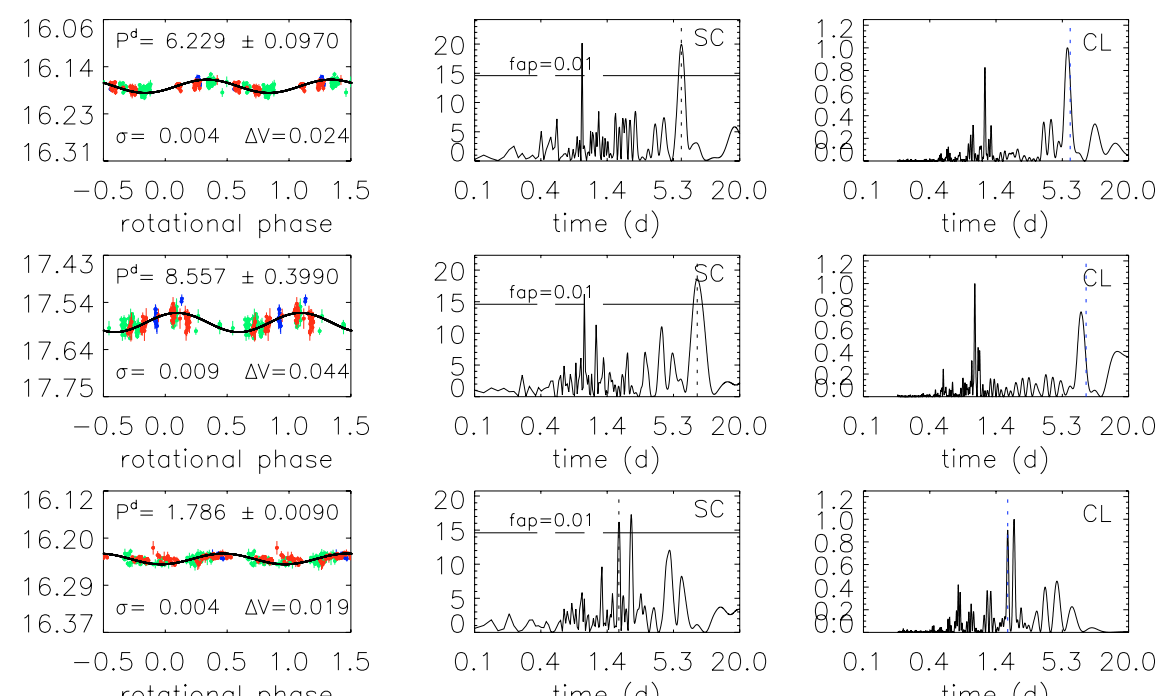

$\begin{array}{lllll}0.1 & 0.4 & 1.4 & 5.3 & 20.0\end{array}$ time (d)
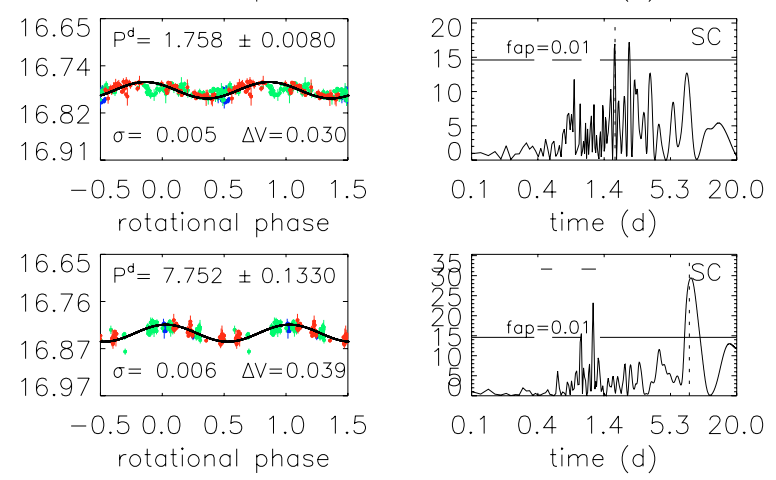

$\begin{array}{lllll}0.1 & 0.4 & 1.4 & 5.3 & 20.0\end{array}$ time (d)
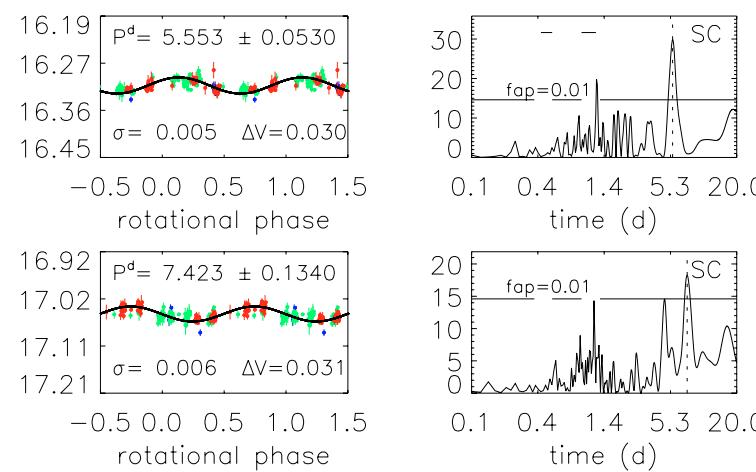

$\begin{array}{lllll}0.1 & 0.4 & 1.4 & 5.3 & 20.0\end{array}$ time (d)
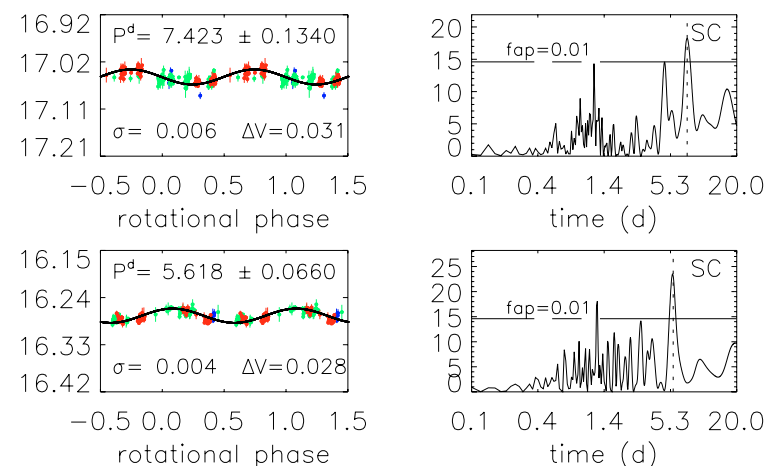

$\begin{array}{lllll}0.1 & 0.4 & 1.4 & 5.3 & 20.0\end{array}$ time (d)
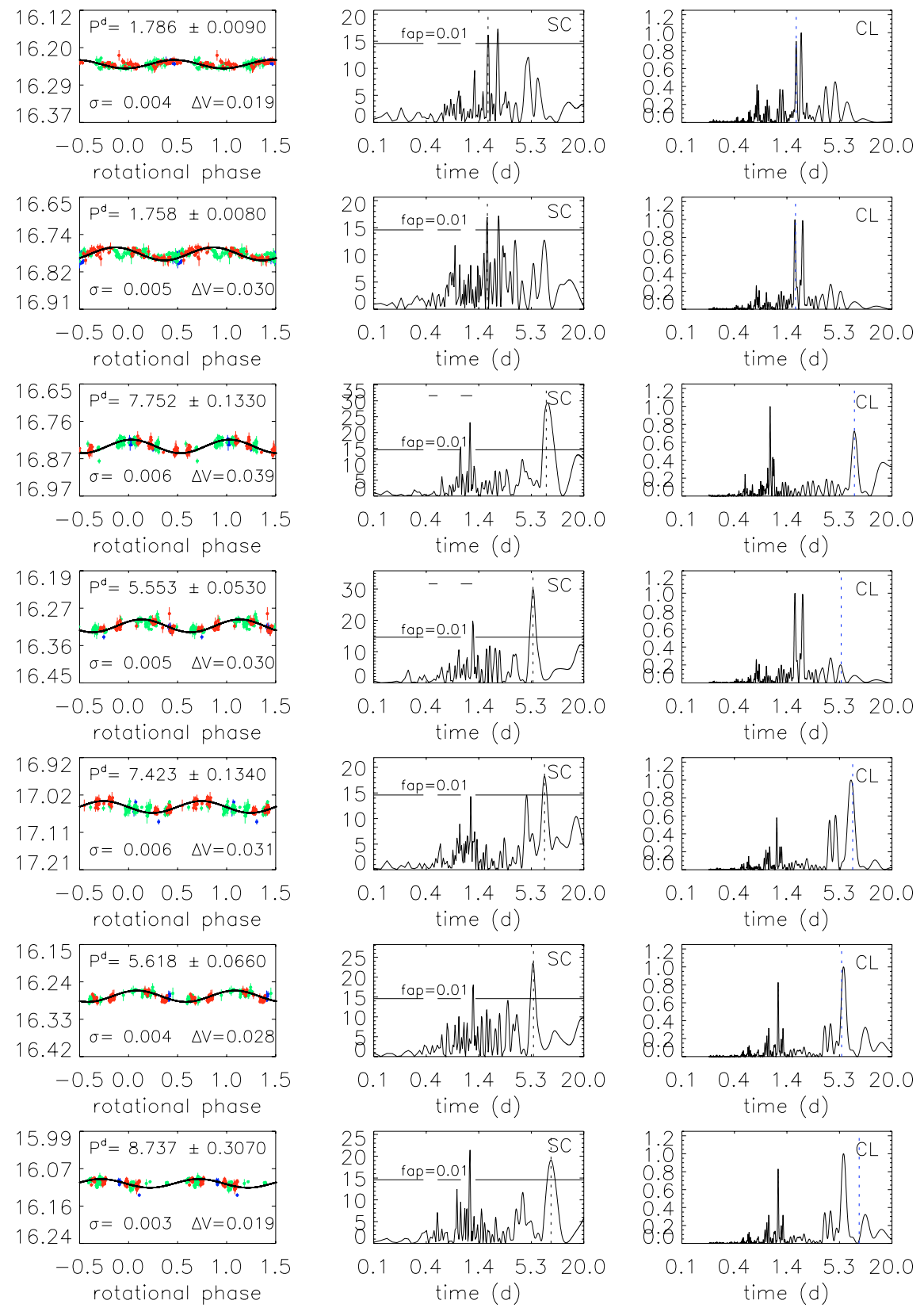
S. Messina et al.: Rotation and variability in NGC 2099, Online Material p 13
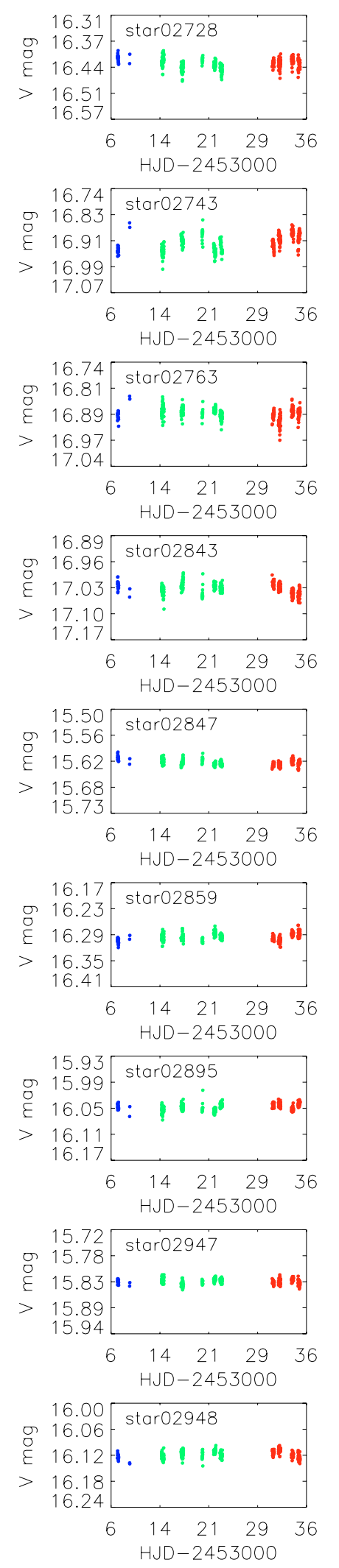
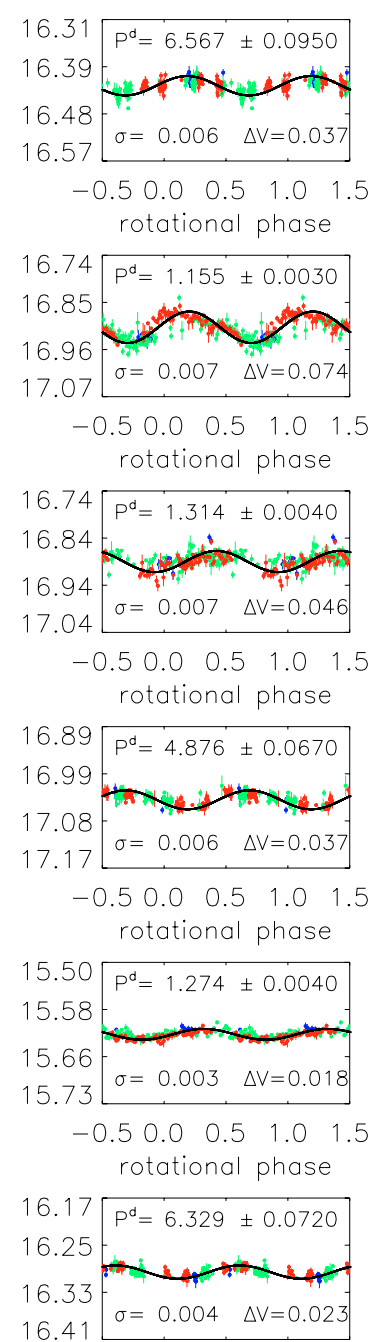

$\begin{array}{lllll}-0.5 & 0.0 & 0.5 & 1.0 & 1.5\end{array}$ rotational phase
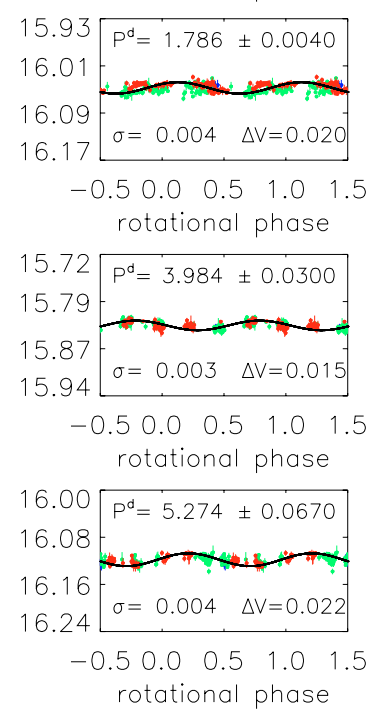
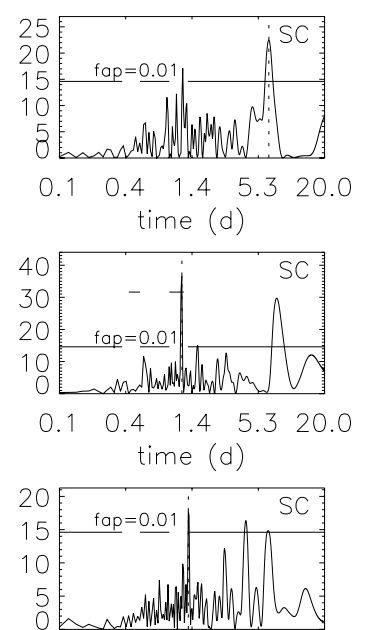

$\begin{array}{lllll}0.1 & 0.4 & 1.4 & 5.3 & 20.0\end{array}$ time (d)
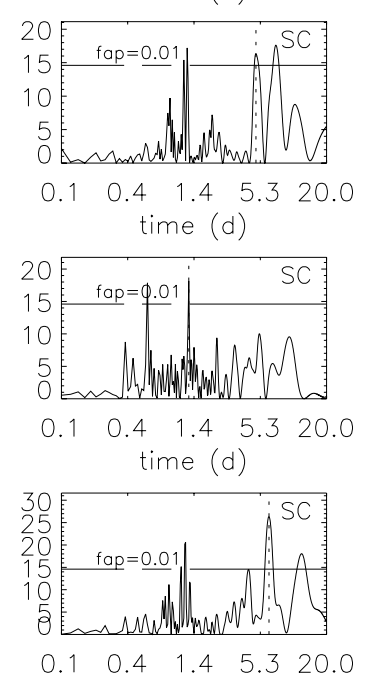
time (d)
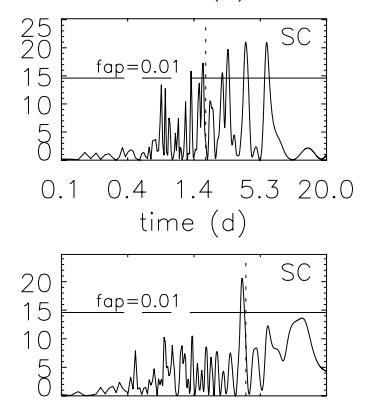

$\begin{array}{lllll}0.1 & 0.4 & 1.4 & 5.3 & 20.0\end{array}$ time (d)

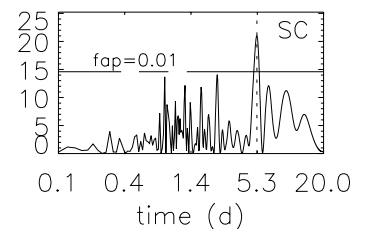

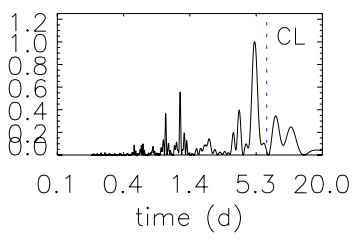
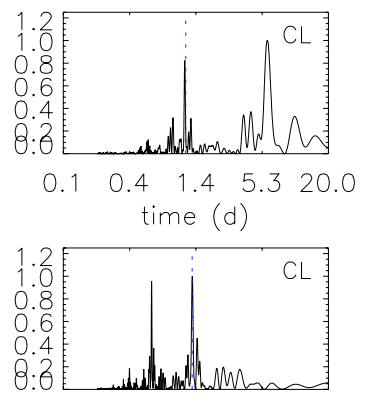

$\begin{array}{lllll}0.1 & 0.4 & 1.4 & 5.3 & 20.0\end{array}$ time (d)
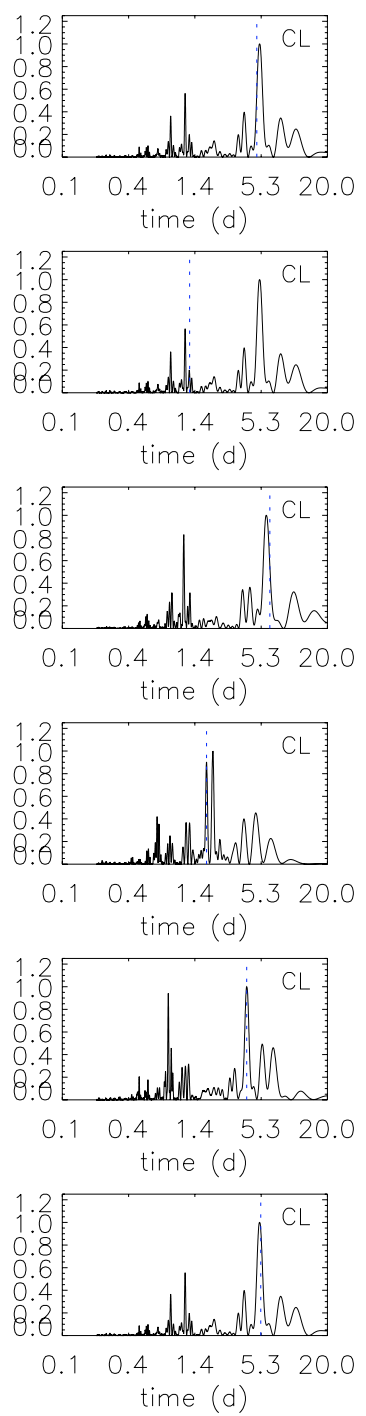

Fig. 19. As in Fig. 17. 
S. Messina et al.: Rotation and variability in NGC 2099, Online Material p 14
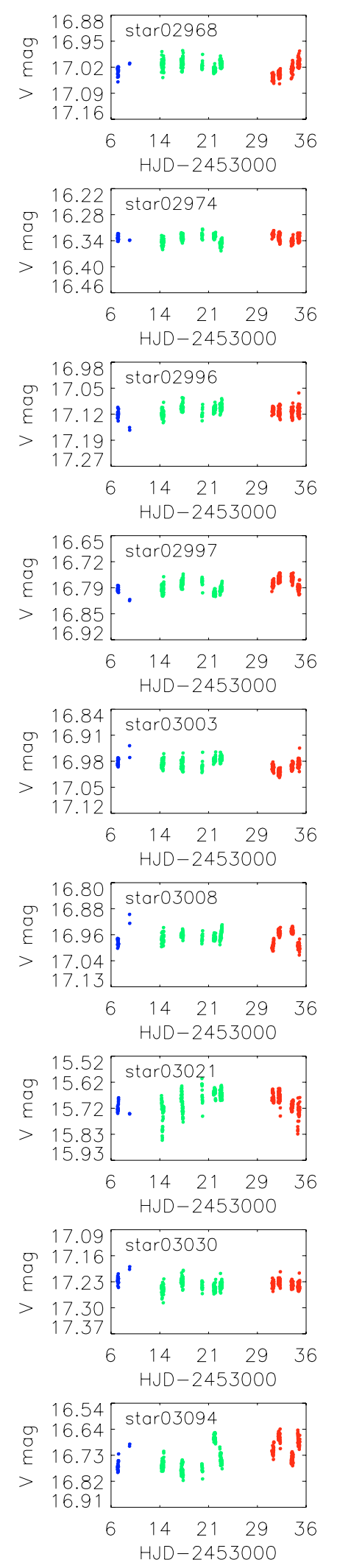

Fig. 20. As in Fig. 17.
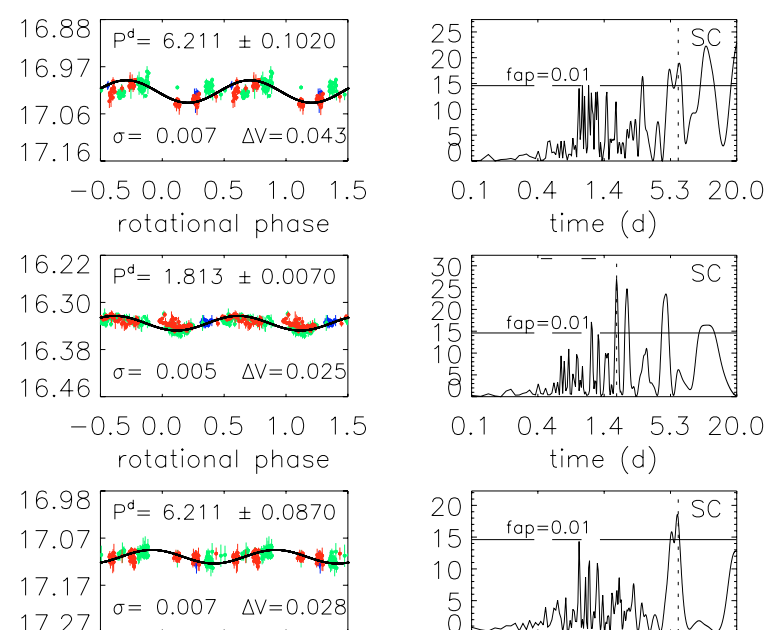

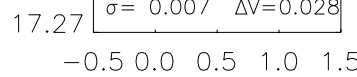
rotational phase

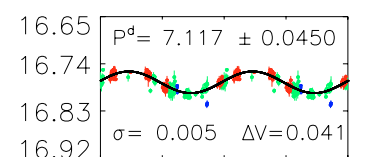

$16.92 \quad \sigma=0.005 \Delta V=0.041$

$\begin{array}{lllll}-0.5 & 0.0 & 0.5 & 1.0 & 1.5\end{array}$ rotational phase
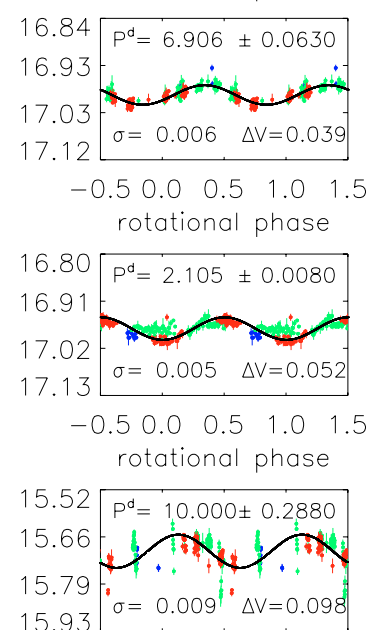

$\begin{aligned} & 15.79 \\ & 15.93\end{aligned}{ }^{\circ} \sigma=0.009^{\circ} " \Delta V=0.098$

$\begin{array}{lllll}-0.5 & 0.0 & 0.5 & 1.0 & 1.5\end{array}$ rotational phase
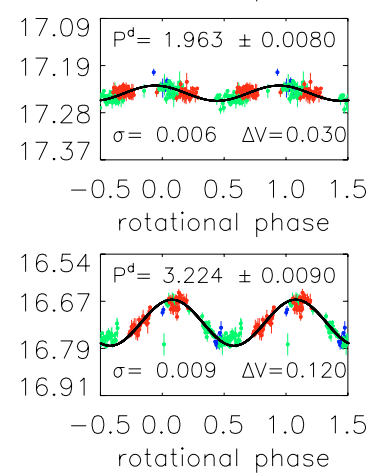
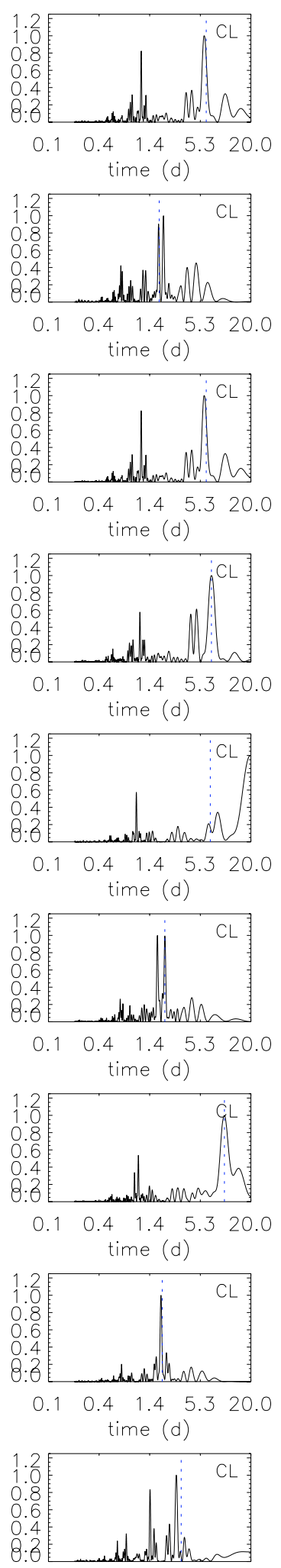

$\begin{array}{lllll}0.1 & 0.4 & 1.4 & 5.3 & 20.0\end{array}$ time (d) 
S. Messina et al.: Rotation and variability in NGC 2099, Online Material p 15
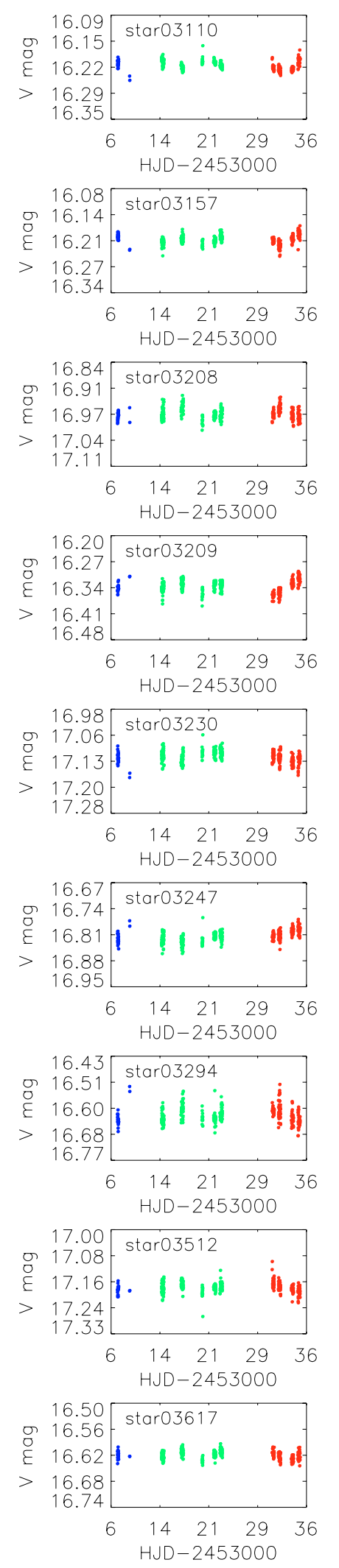
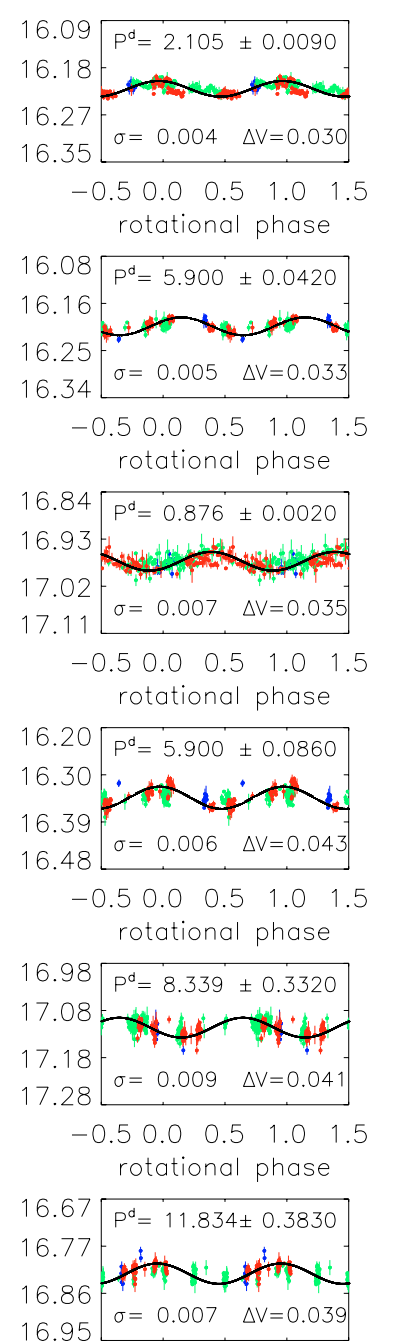

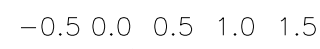
rotational phase
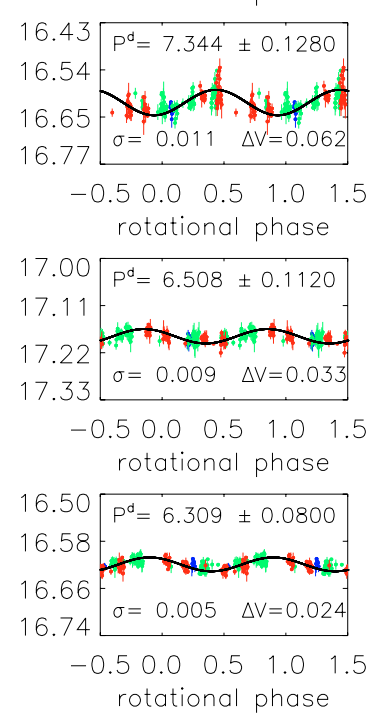
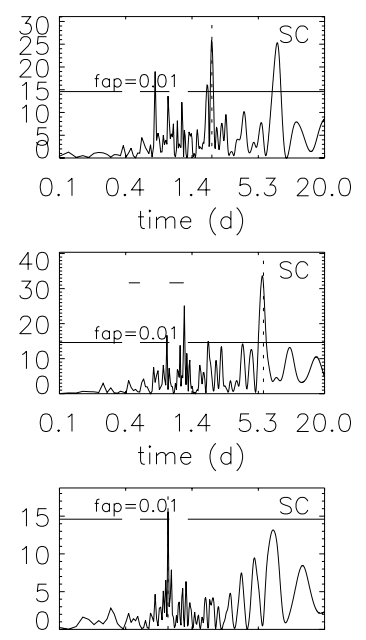

$\begin{array}{lllll}0.1 & 0.4 & 1.4 & 5.3 & 20.0\end{array}$ time (d)
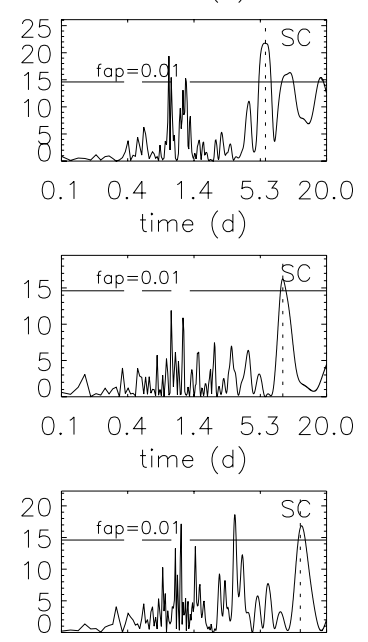

$\begin{array}{lllll}0.1 & 0.4 & 1.4 & 5.3 & 20.0\end{array}$ time (d)
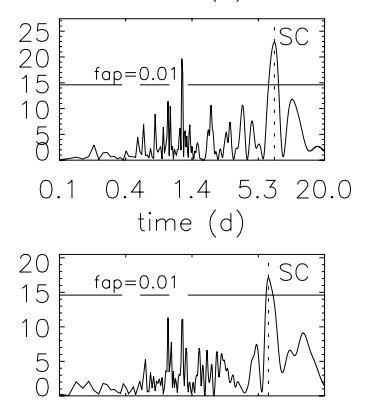

$\begin{array}{lllll}0.1 & 0.4 & 1.4 & 5.3 & 20.0\end{array}$ time (d)

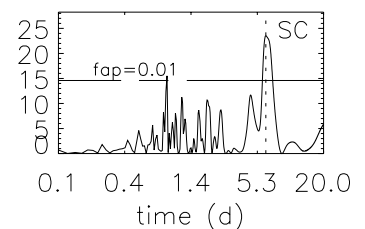

time (d)
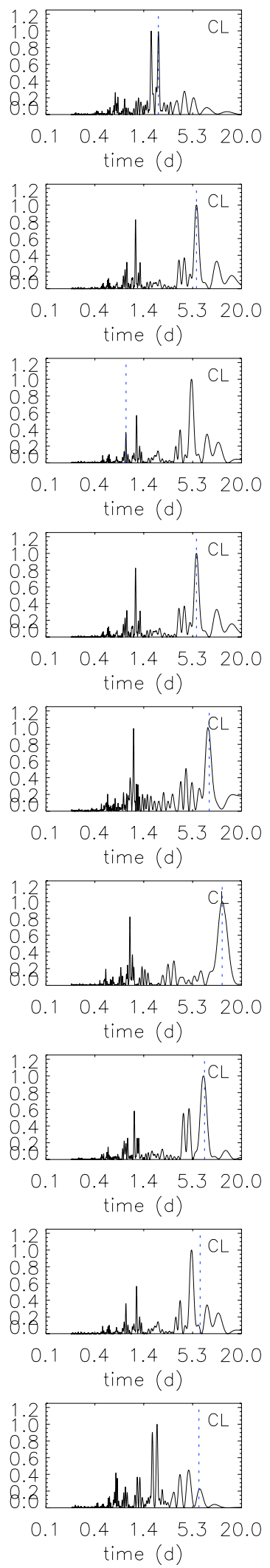

Fig. 21. As in Fig. 17. 
S. Messina et al.: Rotation and variability in NGC 2099, Online Material p 16
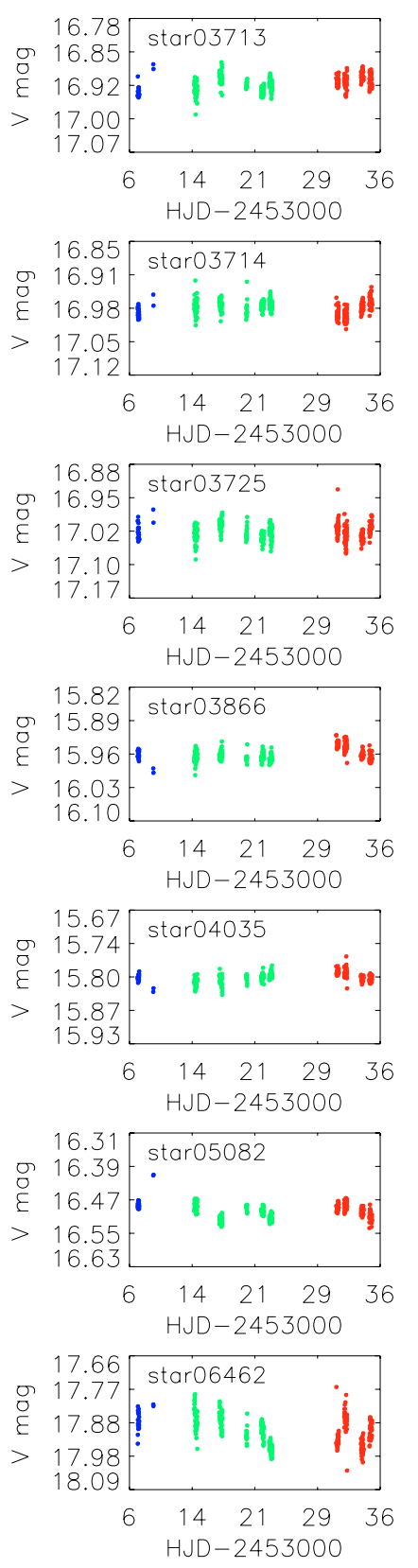

Fig. 22. As in Fig. 17.
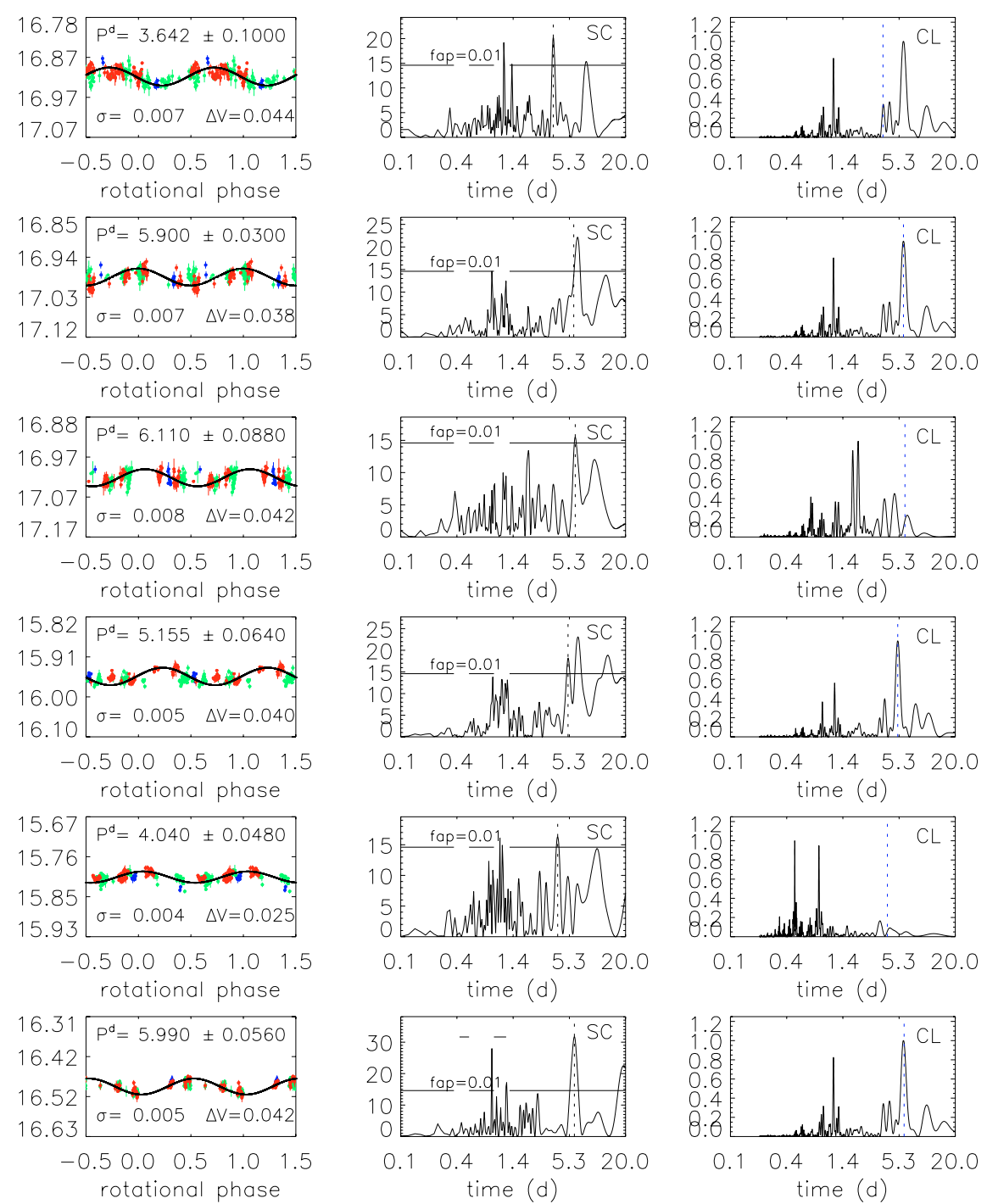

$\begin{array}{lllll}0.1 & 0.4 & 1.4 & 5.3 & 20.0\end{array}$ time (d)
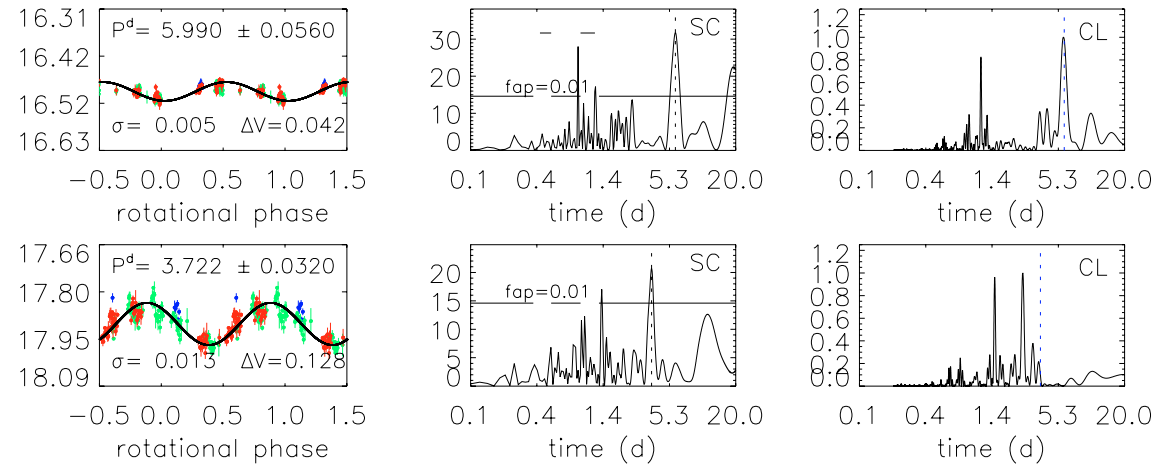
S. Messina et al.: Rotation and variability in NGC 2099, Online Material p 17
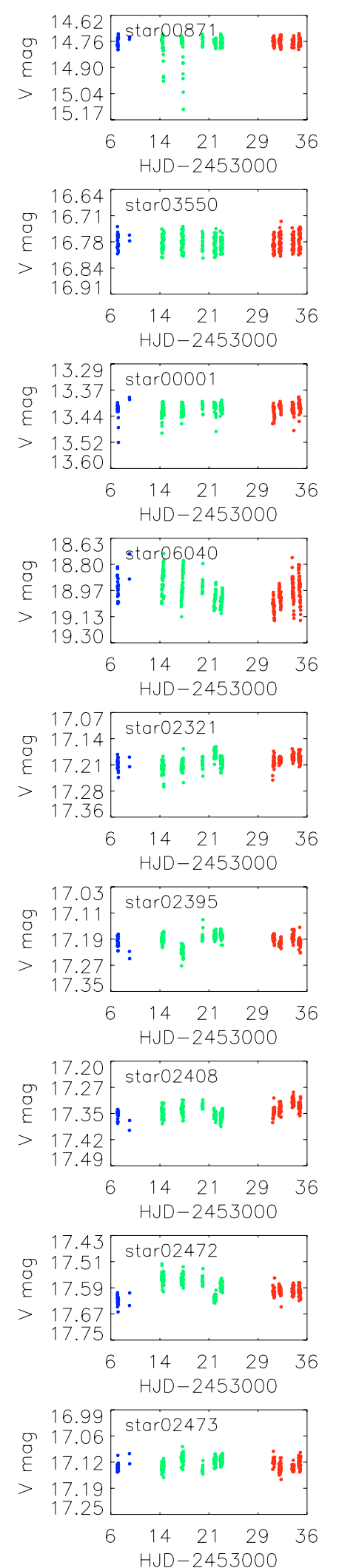
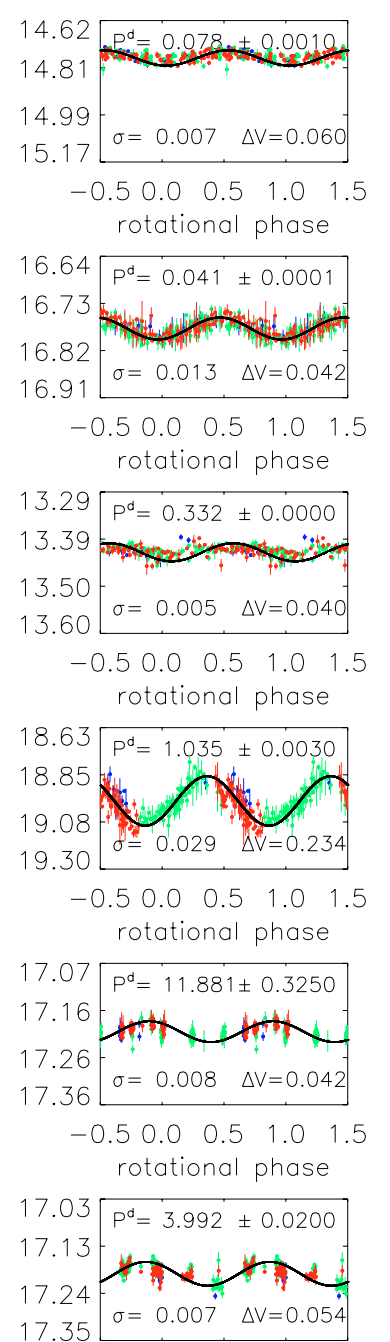

$\begin{array}{lllll}-0.5 & 0.0 & 0.5 & 1.0 & 1.5\end{array}$ rotational phase
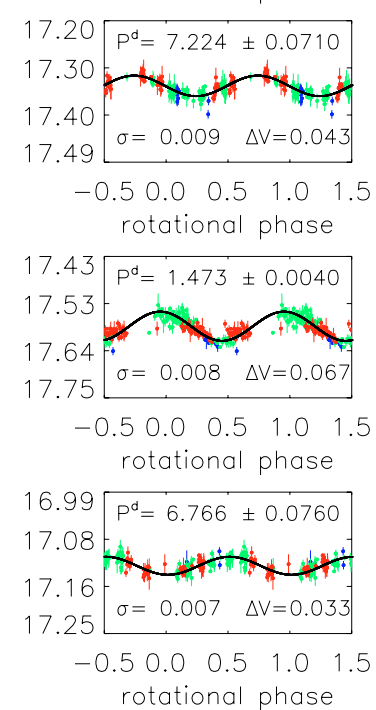
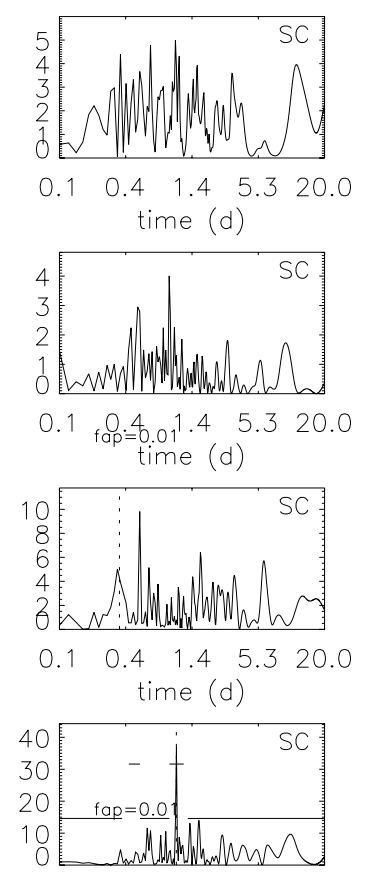

$\begin{array}{lllll}0.1 & 0.4 & 1.4 & 5.3 & 20.0\end{array}$ time (d)
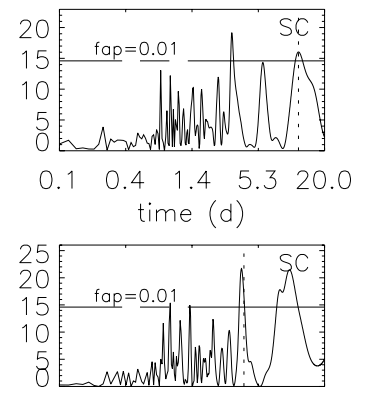

$\begin{array}{lllll}0.1 & 0.4 & 1.4 & 5.3 & 20.0\end{array}$ time (d)

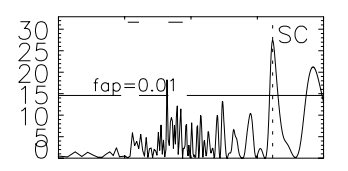

$\begin{array}{lllll}0.1 & 0.4 & 1.4 & 5.3 & 20.0\end{array}$ time (d)
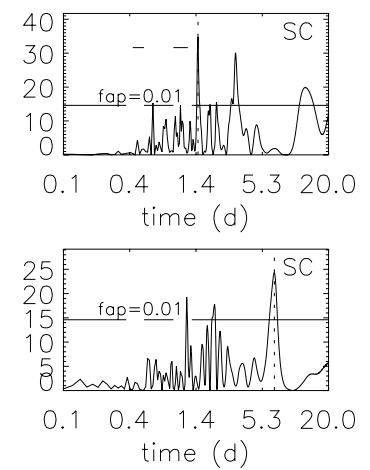
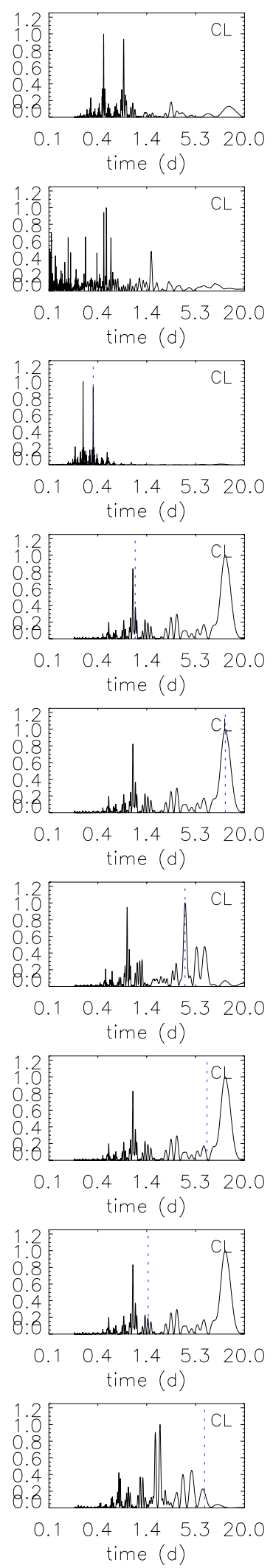

Fig. 23. K-type periodic candidate cluster members. From left panel: V-band time series; phased light curve; Scargle and CLEAN periodogram. See Sect. 3.3 for a detailed description. 
S. Messina et al.: Rotation and variability in NGC 2099, Online Material p 18
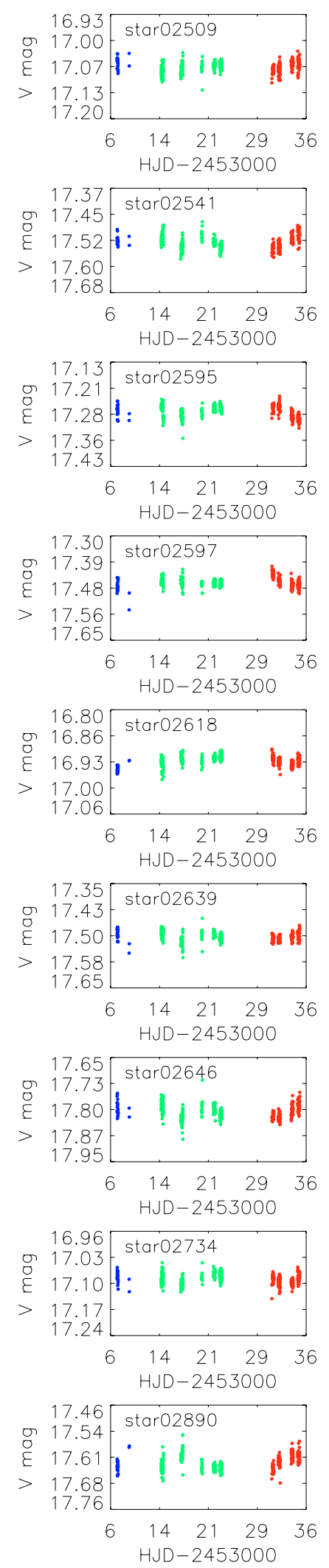

Fig. 24. As in Fig. 23.
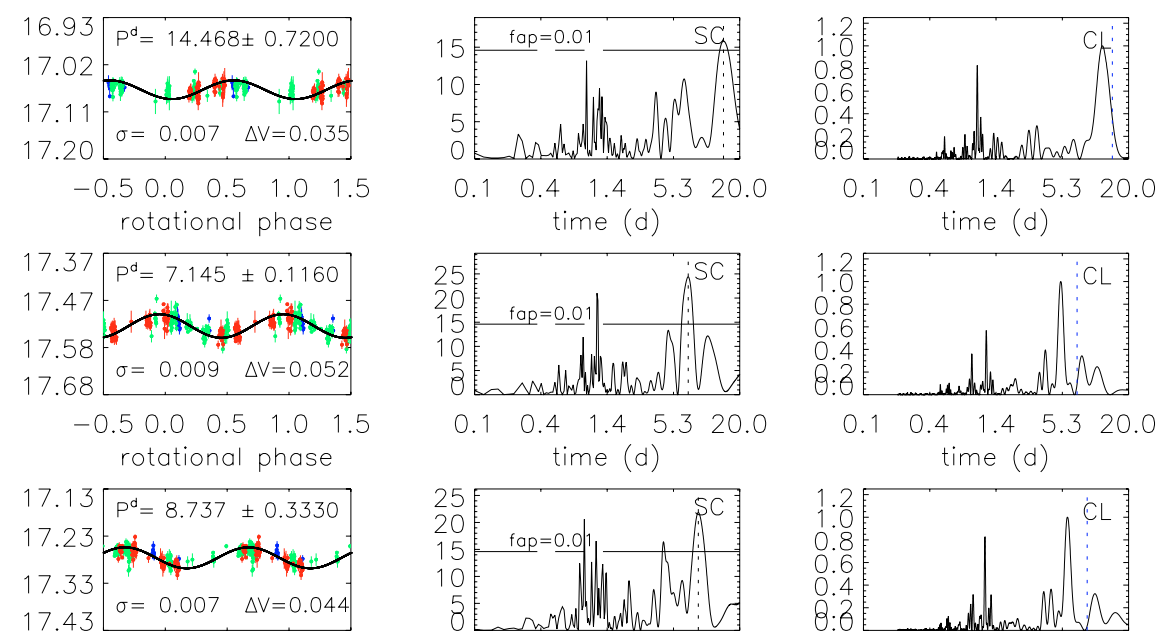

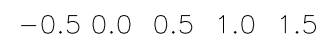
rotational phase
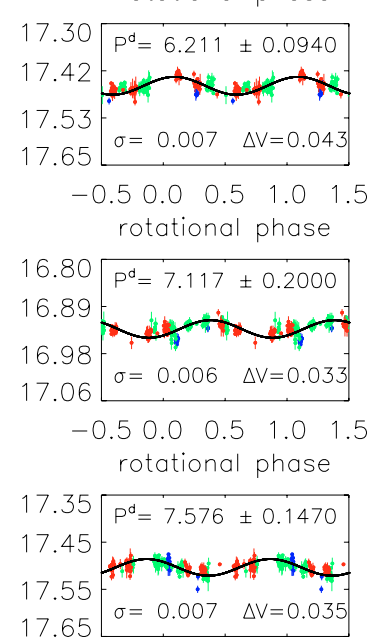

$\begin{array}{lllll}-0.5 & 0.0 & 0.5 & 1.0 & 1.5\end{array}$ rotational phase
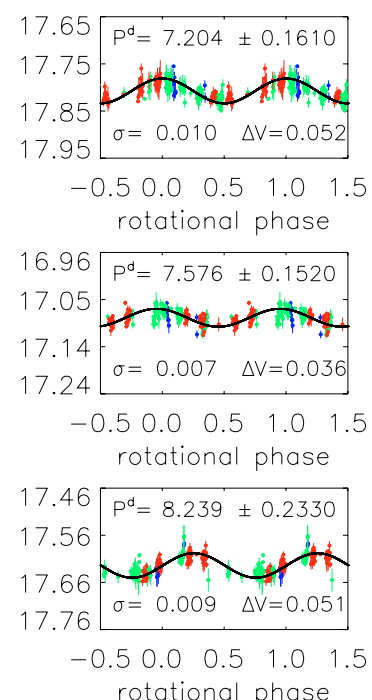

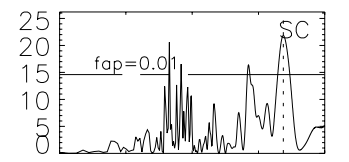

$\begin{array}{lllll}0.1 & 0.4 & 1.4 & 5.3 & 20.0\end{array}$ time (d)
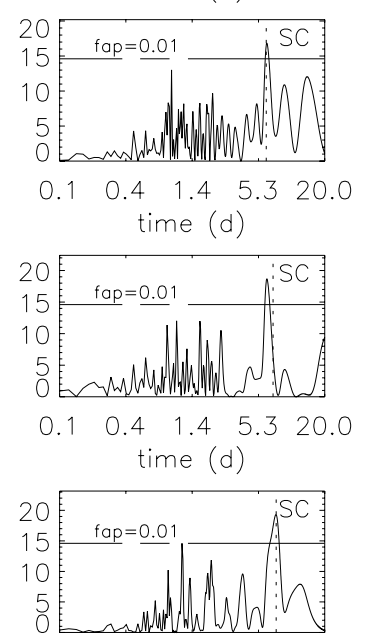

$\begin{array}{lllll}0.1 & 0.4 & 1.4 & 5.3 & 20.0\end{array}$ time (d)

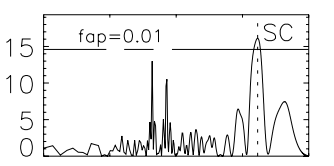

$\begin{array}{lllll}0.1 & 0.4 & 1.4 & 5.3 & 20.0\end{array}$ time (d)

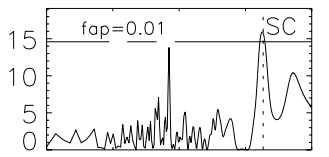

$\begin{array}{lllll}0.1 & 0.4 & 1.4 & 5.3 & 20.0\end{array}$ time (d)

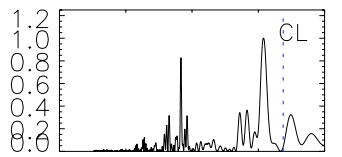

$\begin{array}{lllll}0.1 & 0.4 & 1.4 & 5.3 & 20.0\end{array}$ time (d)
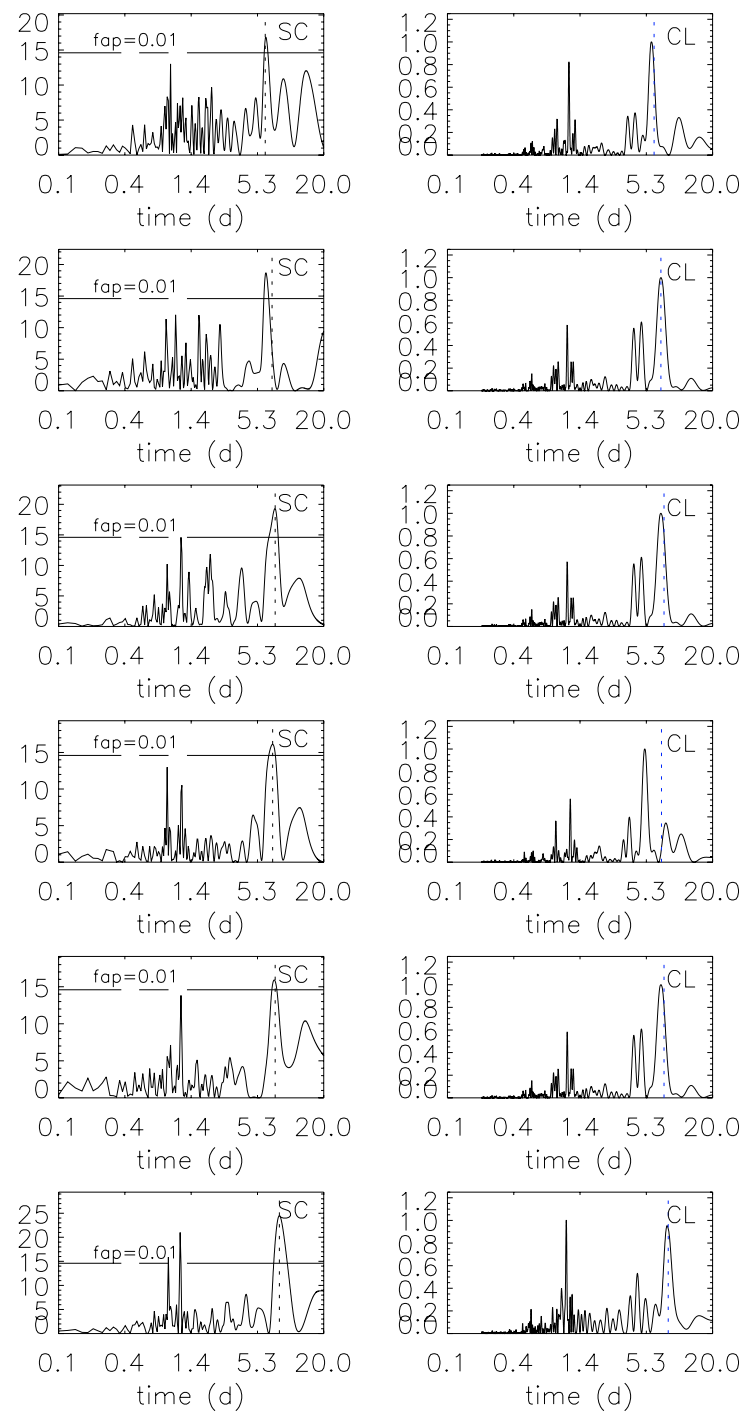
S. Messina et al.: Rotation and variability in NGC 2099, Online Material p 19
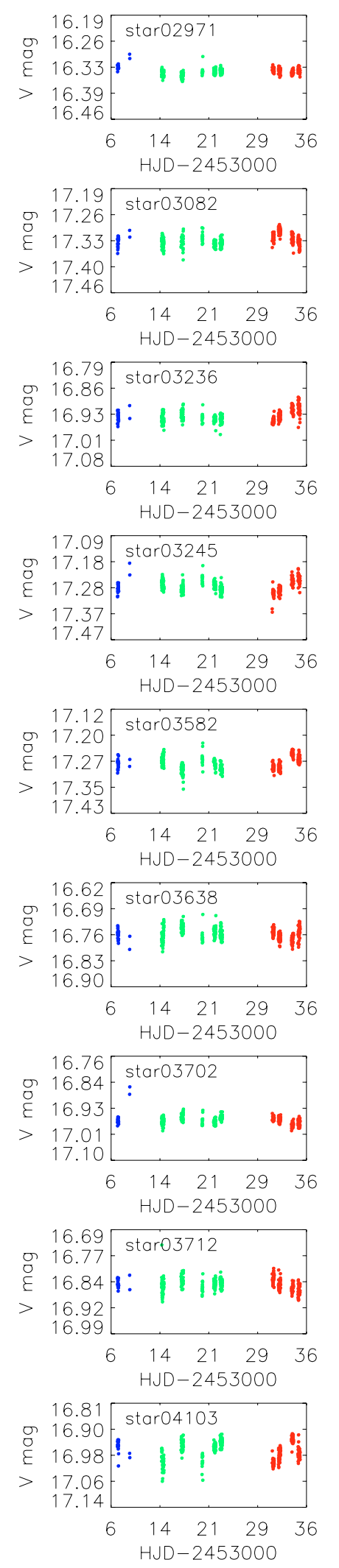
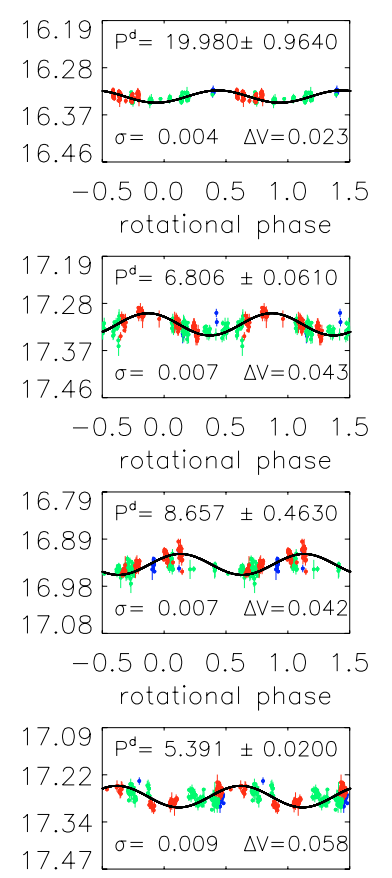

$17.47 \quad \sigma=0.009 \quad \Delta V=0.058$

rotational phase
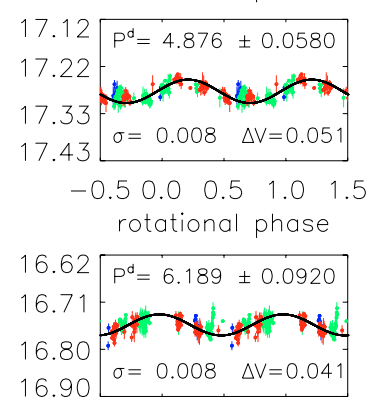

$\begin{array}{lllll}-0.5 & 0.0 & 0.5 & 1.0 & 1.5\end{array}$ rotational phase
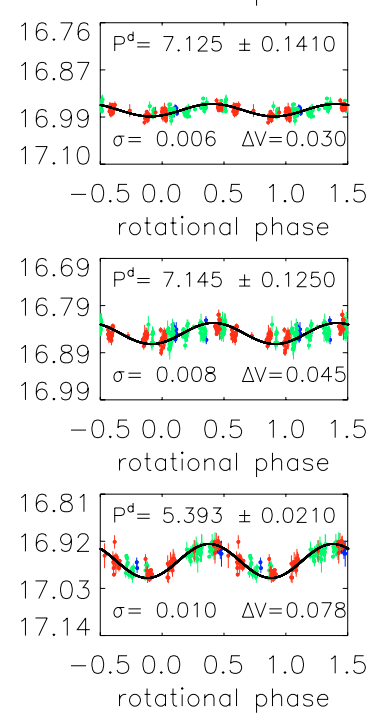
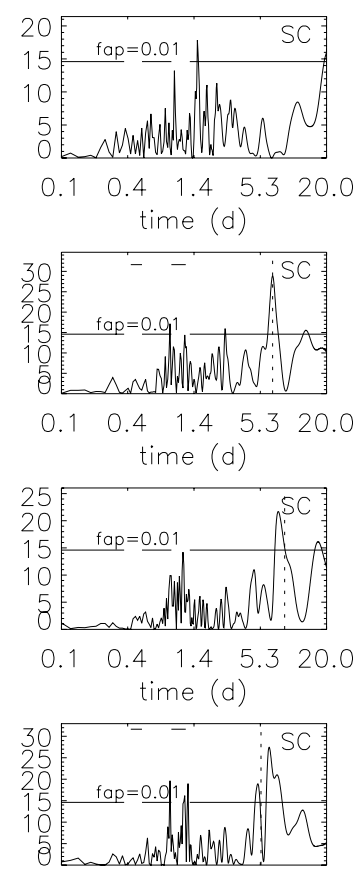

$\begin{array}{lllll}0.1 & 0.4 & 1.4 & 5.3 & 20.0\end{array}$ time (d)
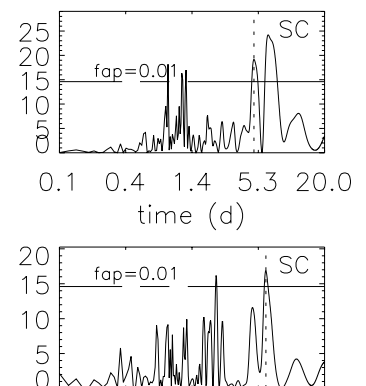

$\begin{array}{lllll}0.1 & 0.4 & 1.4 & 5.3 & 20.0\end{array}$ time (d)
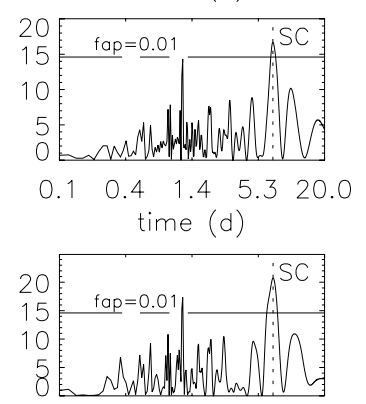

$\begin{array}{lllll}0.1 & 0.4 & 1.4 & 5.3 & 20.0\end{array}$ time (d)

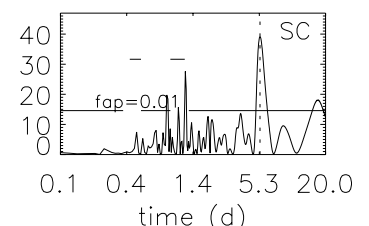

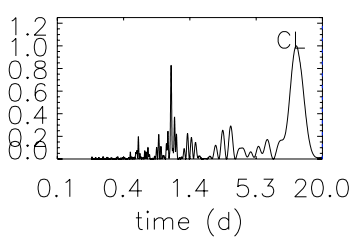
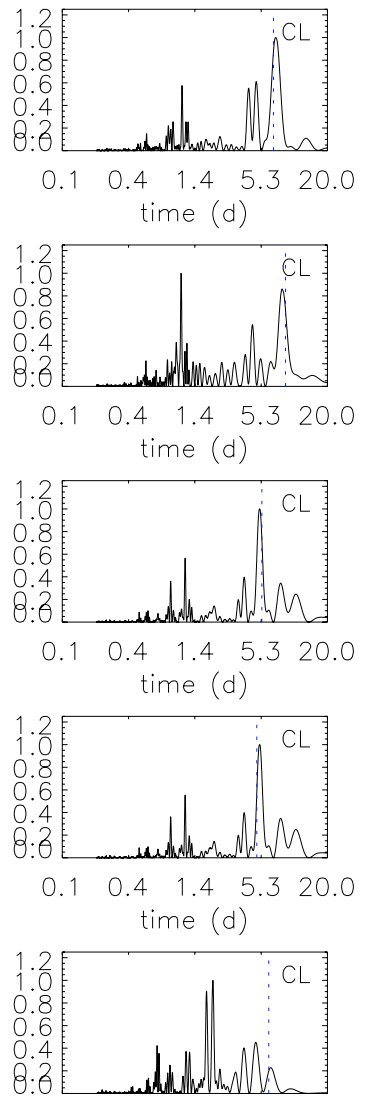

$\begin{array}{lllll}0.1 & 0.4 & 1.4 & 5.3 & 20.0\end{array}$ time (d)
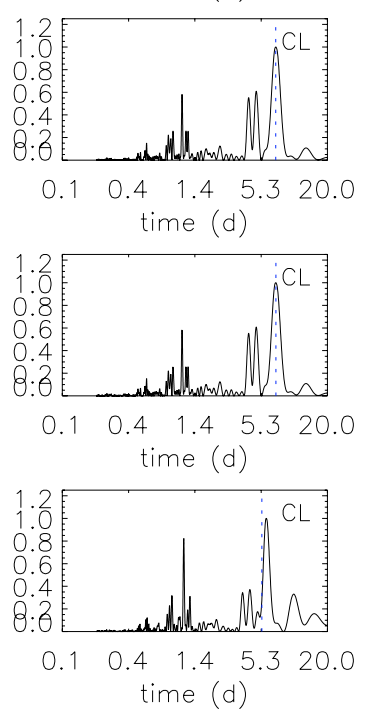

Fig. 25. As in Fig. 23. 
S. Messina et al.: Rotation and variability in NGC 2099, Online Material p 20
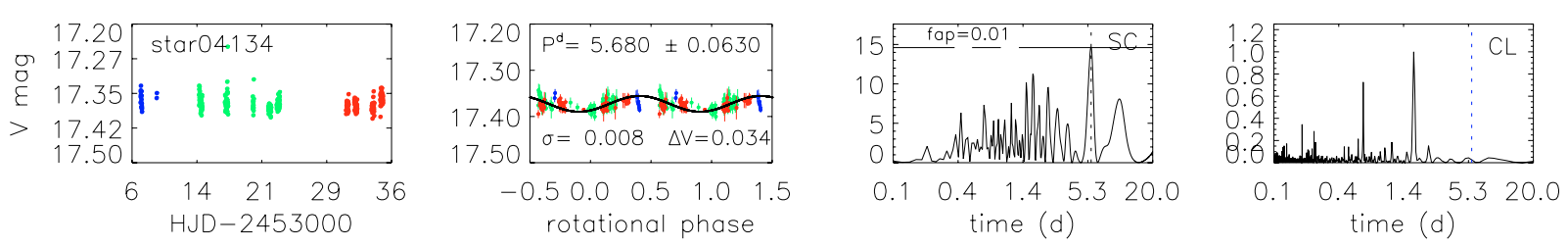

Fig. 26. As in Fig. 23. 